Maskless, Resistless Ion Beam Lithography Processes

by

\title{
Qing Ji
}

B.S. (University of Science and Technology of China) 1993

M.A. (University of California, Berkeley) 1998

A dissertation submitted in partial satisfaction of the

requirements for the degree of

Doctor of Philosophy

in

Engineering - Electrical Engineering

and Computer Sciences

in the

GRADUATE DIVISION

of the

UNIVERSITY OF CALIFORNIA, BERKELEY

\author{
Committee in charge: \\ Professor Tsu-Jae King, Chair \\ Professor Andrew Neureuther \\ Professor Ka-Ngo Leung
}

Spring 2003 
The dissertation of Qing Ji is approved:

Professor Tsu-Jae King, Chair Date

Professor Andrew Neureuther

Date

Professor Ka-Ngo Leung

Date

University of California, Berkeley

Spring 2003 
Maskless, Resistless Ion Beam Lithography Processes

Copyright (C) 2003

by

Qing Ji

All rights reserved 


\title{
Abstract
}

\section{Maskless, Resistless Ion Beam Lithography Processes}

by

Qing Ji

\author{
Doctoral of Philosophy in Engineering - \\ Electrical Engineering and Computer Sciences \\ University of California, Berkeley \\ Professor Tsu-Jae King, Chair
}

As the dimensions of semiconductor devices are scaled down, in order to achieve higher levels of integration, optical lithography will no longer be sufficient for the needs of the semiconductor industry. Alternative next-generation lithography (NGL) approaches, such as extreme ultra-violet (EUV), X-ray, electron-beam, and ion projection lithography face some challenging issues with complicated mask technology and low throughput. Among the four major alternative NGL approaches, ion beam lithography is the only one that can provide both maskless and resistless patterning. As such, it can potentially make nano-fabrication much simpler. This thesis investigates a focused ion beam system for maskless, resistless patterning that can be made practical for highvolume production.

In order to achieve maskless, resistless patterning, the ion source must be able to produce a variety of ion species. The compact FIB system being developed uses a multicusp plasma ion source, which can generate ion beams of various elements, such as 
$\mathrm{O}_{2}^{+}, \mathrm{BF}_{2}^{+}, \mathrm{P}^{+}$etc., for surface modification and doping applications. With optimized source condition, around $85 \%$ of $\mathrm{BF}_{2}^{+}$, over $90 \%$ of $\mathrm{O}_{2}^{+}$and $\mathrm{P}^{+}$have been achieved.

The brightness of the multicusp-plasma ion source is a key issue for its application to maskless ion beam lithography. It can be substantially improved by optimizing the source configuration and extractor geometry. Measured brightness of $2 \mathrm{keV} \mathrm{He}^{+}$beam is as high as $440 \mathrm{~A} / \mathrm{cm}^{2} \cdot \mathrm{Sr}$, which represents a $30 \times$ improvement over prior work.

Direct patterning of $\mathrm{Si}$ thin film using a focused $\mathrm{O}_{2}{ }^{+}$ion beam has been investigated. A thin surface oxide film can be selectively formed using $3 \mathrm{keV} \mathrm{O}_{2}{ }^{+}$ions with the dose of $10^{15} \mathrm{~cm}^{-2}$. The oxide can then serve as a hard mask for patterning of the Si film. The process flow and the experimental results for directly patterned poly- $\mathrm{Si}$ features are presented. The formation of shallow $p n$-junctions in bulk silicon wafers by scanning focused $\mathrm{P}^{+}$beam implantation at $5 \mathrm{keV}$ is also presented. With implantation dose of around $10^{16} \mathrm{~cm}^{-2}$, the electron concentration is about $2.5 \times 10^{18} \mathrm{~cm}^{-3}$ and electron mobility is around $200 \mathrm{~cm}^{2} / \mathrm{V}$.s. To demonstrate the suitability of scanning FIB lithography for the manufacture of integrated circuit devices, SOI MOSFET fabrication using the maskless, resistless ion beam lithography is demonstrated.

An array of microcolumns can be built by stacking multi-aperture electrode and insulator layers. Because the multicusp plasma source can achieve uniform ion density over a large area, it can be used in conjunction with the array of microcolumns, for massively parallel FIB processing to achieve reasonable exposure throughput. 
The dissertation abstract of Qing Ji is approved:

Professor Tsu-Jae King Date

Committee Chair 
To my family 


\section{Table of Contents}

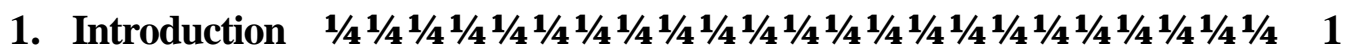

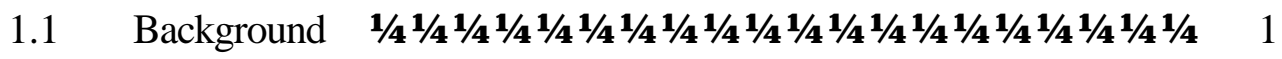

1.2 Next generation lithography techniques ..................... 3

1.2.1 Extreme UltraViolet (EUV) lithography................ 3

1.2.2 X-ray lithography................................ 4

1.2.3 Electron beam lithography $\ldots \ldots \ldots \ldots \ldots \ldots \ldots \ldots \ldots \ldots \ldots$

1.2.4 Ion beam lithography $\ldots \ldots \ldots \ldots \ldots \ldots \ldots \ldots \ldots \ldots \ldots . . \ldots \ldots$

1.3 Maskless, resistless ion beam lithography ................... 9

1.3.1 Motivation .................................... 9

1.3.2 Maskless, resistless ion beam lithography processes..... 10

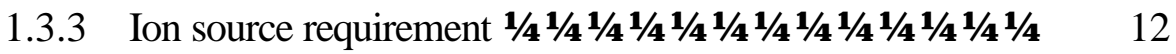

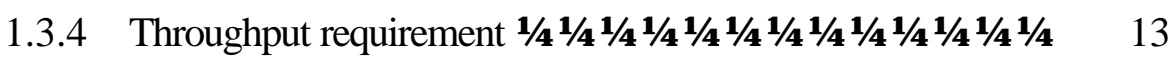

References ......................................... 15

2. Multicusp Plasma Ion source for MRIBL $\ldots \ldots \ldots \ldots \ldots \ldots \ldots \ldots .20$

$2.1 \quad$ Overview of FIB $\ldots \ldots \ldots \ldots \ldots \ldots \ldots \ldots \ldots \ldots \ldots \ldots \ldots \ldots \ldots \ldots, 20$

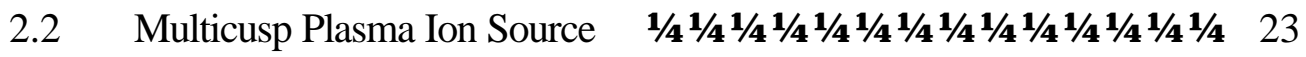

2.2.1 Principle of multicusp plasma source $\quad \ldots \ldots \ldots \ldots \ldots \ldots \ldots 23$

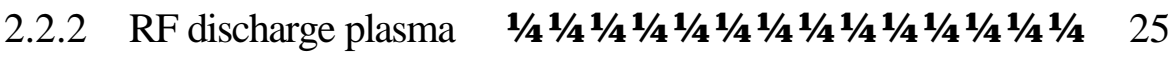

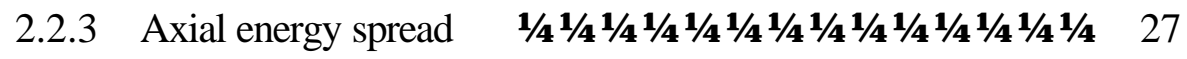


2.3 Current density of Multicusp Plasma Ion source

2.3.1 Extraction from a plasma source

2.3.2 Current density as a function of extraction voltage

2.3.3 Current density as a function of RF power

2.3.4 Current density as a function of source size .

2.4 Sources with external antenna .

References

3. Beam Brightness of Multicusp-Plasma Ion Source

3.1 Brightness

3.1.1 Source brightness

3.1.2 Beam brightness

3.1.3 Comparison of brightness of different source

3.1.4 Requirement of brightness

3.2 Spectral brightness measurement

3.2.1 Experimental setup

3.2.2 Results and discussion 46

3.3 Beam brightness measurement

3.3.1 Increasing total beam current 50

3.3.2 Reducing focused beam spot size

3.3.3 Experimental setup

3.3.4 Results and Discussion .... 


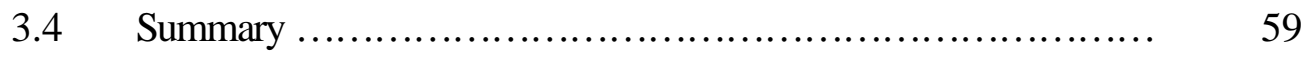

References ..................................................... 61

4. Ion Optics for Maskless, Resistless Ion Beam Lithography System ... 63

4.1 Electrostatic lenses .......................................... 63

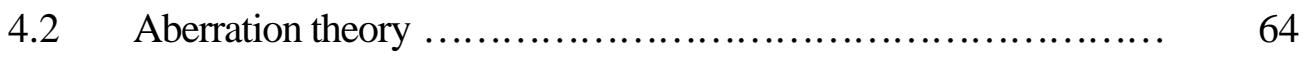

4.2.1 Spherical aberration .................................. 65

4.2.2 Coma aberration ...................................... 66

4.2.3 Field curvature and astigmatism …..................... 67

4.2.4 Distortion ............................................... 68

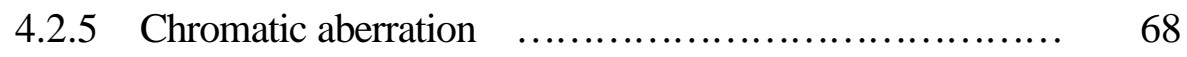

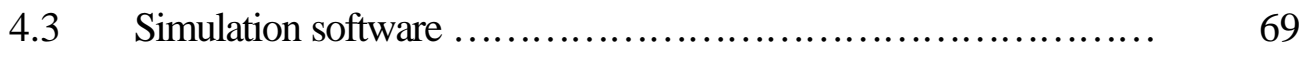

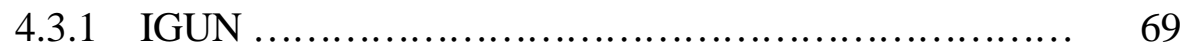

4.3.2 Munro Code ............................................... $\quad 70$

Single lens system ...........................................

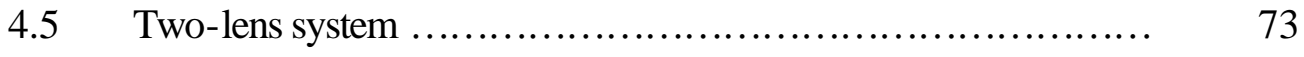

4.5.1 IGUN simulation results .............................

4.5.2 Munro's code simulation results .......................... 76

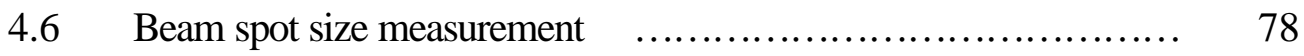

4.6.1 Column fabrication .................................. 78

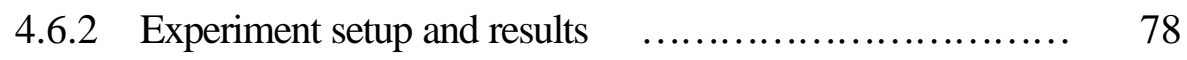

$4.7 \quad$ Improvement of ion optics design ................................ $\quad 80$ 
4.7.1 Adding limiting aperture $\ldots \ldots \ldots \ldots \ldots \ldots \ldots \ldots \ldots \ldots \ldots . . \ldots \ldots$

4.7.2 Reducing size of extraction aperture .................... 80

4.7.2.1 Fabrication of a $5 \mu \mathrm{m}$ counterbored aperture ....... 81

4.7.2.2 Extraction current with smaller extraction aperture ... 83

References .............................................. 85

5. Maskless, resistless Ion Beam Lithography Processes ............. 87

5.1 Production of various ion species .......................... 87

5.1.1 Phosphorus ions: $\mathrm{P}_{\mathrm{x}}{ }^{+}$and $\mathrm{P}_{\mathrm{x}}{ }^{-}$ions $(\mathrm{x}=1,2,3,4,5) \ldots \ldots \ldots \quad 88$

5.1.2 Boron ions: $\mathrm{BF}_{\mathrm{x}}{ }^{+}$and $\mathrm{BF}_{\mathrm{x}}{ }^{-}$ions $(\mathrm{x}=1,2,3,4,5) \ldots \ldots \ldots . .90$

5.1.3 Oxygen ions: $\mathrm{O}_{\mathrm{x}}{ }^{+}$and $\mathrm{O}_{\mathrm{x}}{ }^{-}$ions $(\mathrm{x}=1,2) \ldots \ldots \ldots \ldots \ldots \ldots . \ldots 3$

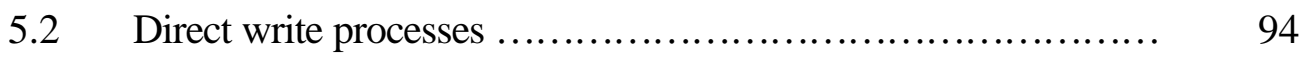

5.2.1 Direct doping using boron and phosphorous beam ........ 94

5.2.2 Surface modification using oxygen beam ................ 96

5.3 SOI MOSFET fabrication using maskless resistless ion beam

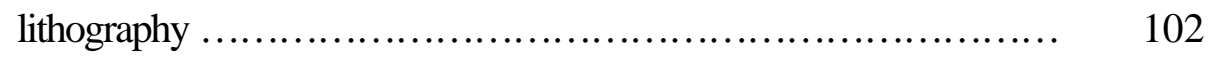

References ............................................. 105

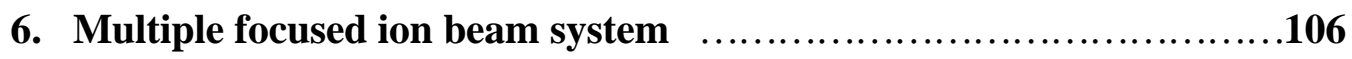

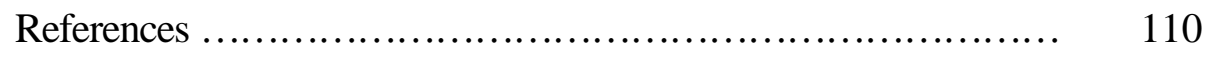

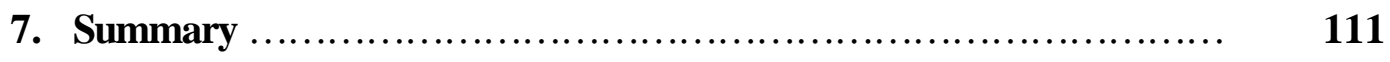




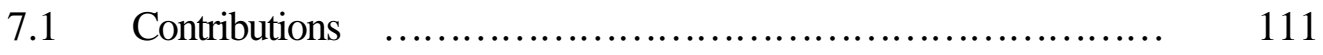

7.2 Suggestions for future work ............................. 113 


\section{Acknowledgments}

I want to thank all the people who helped me through all these years.

To Professor Tsu-Jae King who has always been my role-model, thank you for all your guidance, advice, encouragement, and support... Your feedback and comments on my paper and presentations were always very helpful, and I will continue to work hard in improving my skill in both.

To Professor Ka-Ngo Leung, thank you for giving me the opportunity to work on the project, thank you for all the advice, encouragement and support. Every time when I feel frustrated, I will always remember what you've told me. 'Don't be afraid of losing one battle, as long as one wins the war". I will never give up so easily.

I also want to thank professors Andy Neureuther and Nathan Cheung, for your suggestions on my research.

I want to thank all the staff (present and past) from Plasma and Ion Source Technology Group and Ion Beam Technology program at the Lawrence Berkeley National Laboratory. In particular, I would like to thank Steve Wilde for being so good in making ion sources and accelerator parts. Anything I draw on a scratch paper, you can make it for me. Thank Yvette Lee, Jani Reijonen, Thomas Schenkel, Daniela Wutte, and Don Williams for your helpful discussions; thank Paul Wong, Jerry Fischer, Glenn Jones, and Larry Mill for your mechanical support; thank Tom McVeigh, Chip Kozy, and Pete Rosado for helping me solve all the electrical problems so that I can run experiments; thank Matt Eardley, Ximan Jiang, Audray Chang, thanks for helping me get such good results on experiments; thank Darlene Hawkins, Martha Condon, Tom Gallant, Faye Mitschang for taking care of all those miscellanies that I always missed. 
To Rod Keller, the first person who led me into the charge particle optics field, thank you for your instructions on IGUN; to Eric Munro, Xieqing Zhu, thank for all your technical support and your expertise on electron optics; to Junqiao $\mathrm{Wu}$, thanks for the Hall effect measurement; and to Kin-man Yu, thanks for the RBS measurements.

To Karen Scott, Vinh Ngo, thank you. It was always fun working with you.

To members of Device Group: Xuejue Huang, Yu Cao, Qiang Lu, Peiqi Xuan, Mark Cao, Yee-Chia Yeo, Shiying Xiong, Xiaodong Jin, Min She, Hui Wan, Gang Liu, Jane Xi, Jing He, Hideki Takeuchi, Charles Kuo, Kevin Yang, Pin Su, Yangkyu Choi, Leland Chang, Igor Polishchuk, Pushkar Ranade, Daewon Ha, Jakub Kedzierski, Wen-chin Lee, Nick Lindert, Weidong Liu, Stephen Tang, ..... thank you for your friendship and help. I will always cherish memories of those good times we spent together in 373 Cory.

I would like to thank the Berkeley Microlab staff for providing the tools with which we can do high quality research. In particular, I wish to extend a special thanks to Xiaofan Meng for helping me a lot.

I also would like to acknowledge the support from Defense Advanced Research Projects Agency (DARPA) and US Department of Energy through Lawrence Berkeley Lawrence Berkeley National Laboratory under Contract No. DE-AC03-76SF00098.

Finally, but not lastly, I would like to thank my parents whose constant support and understanding allowed my to stay on with my dreams, plans and journeys.

There are many other people worth mentioning that have been a wonderful addition to me both personally and technically during this research. You know who you are, and lest I forget anyone, I owe you my sincere thanks. 


\section{Chapter 1}

\section{Introduction}

\subsection{Background}

Moore's Law states that the number of devices on a chip doubles every 18 months. ${ }^{1}$ Lithography advancements, increased wafer size and design innovation are three main constituents of the technology improvements that have kept the industry on this pace for more than 30 years. Among these three, lithography is the most important driver since roughly half of the density improvements have been derived from improvements in lithography. 2,3 Without the continuous improvements in lithographic process and equipment technology, personal computers, cell phones, and the Internet would not be in widespread use today. 


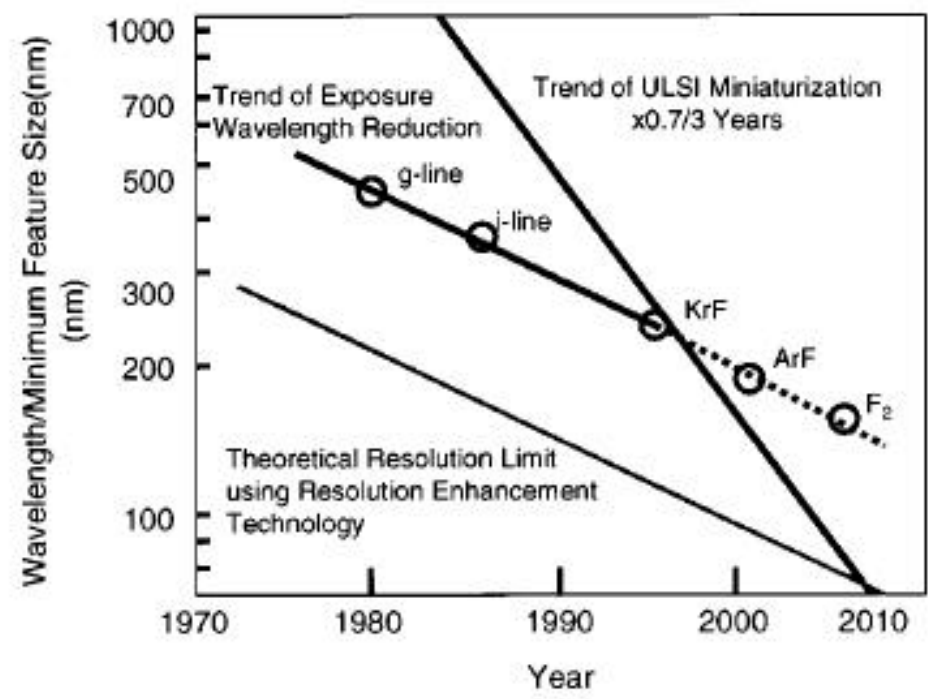

Figure 1.1 Comparison of lithography wavelength trends with IC feature size trend. ${ }^{2}$

To obtain the desired feature size, the semiconductor industry has relied on optical lithography for nearly three decades. The resolution of an optical lithography system is usually expressed in terms of its wavelength $\lambda$ and numerical aperture (NA) as

$$
\text { Resolution }=k_{1} \frac{\lambda}{N A}
$$

where $\mathrm{k}_{1}$ factor is the process-dependent coefficient of the resolution criterion for a diffraction limited lens. As shown in Fig. 1.1, the characteristic wavelength has decreased from G-line at $435 \mathrm{~nm}$ to I-line at $365 \mathrm{~nm}$, which was followed by a switch to excimer laser sources with $\mathrm{KrF}$ at $248 \mathrm{~nm}, \operatorname{ArF}$ at $193 \mathrm{~nm}$, and is currently migrating to $157 \mathrm{~nm}$. Optical lithography is expected to continue as such through the $65 \mathrm{~nm}$ node, ${ }^{3}$ through the application of resolution enhancement techniques such as off-axis illumination (OAI), phase shifting masks (PSM) and optical proximity corrections (OPC). However, optical lithography will be reaching its limits, and the extension of the roadmap requires the development of the next generation lithography technologies. 


\subsection{Next generation lithography techniques}

The next generation lithography techniques have been explored for beyond the $100 \mathrm{~nm}$ lithography node. There are four alternative technologies for a next generation lithography tool: Extreme Ultra Violet (EUV) lithography, X-ray lithography, electron beam lithography and ion beam lithography.

\subsubsection{Extreme UltraViolet (EUV) lithography}

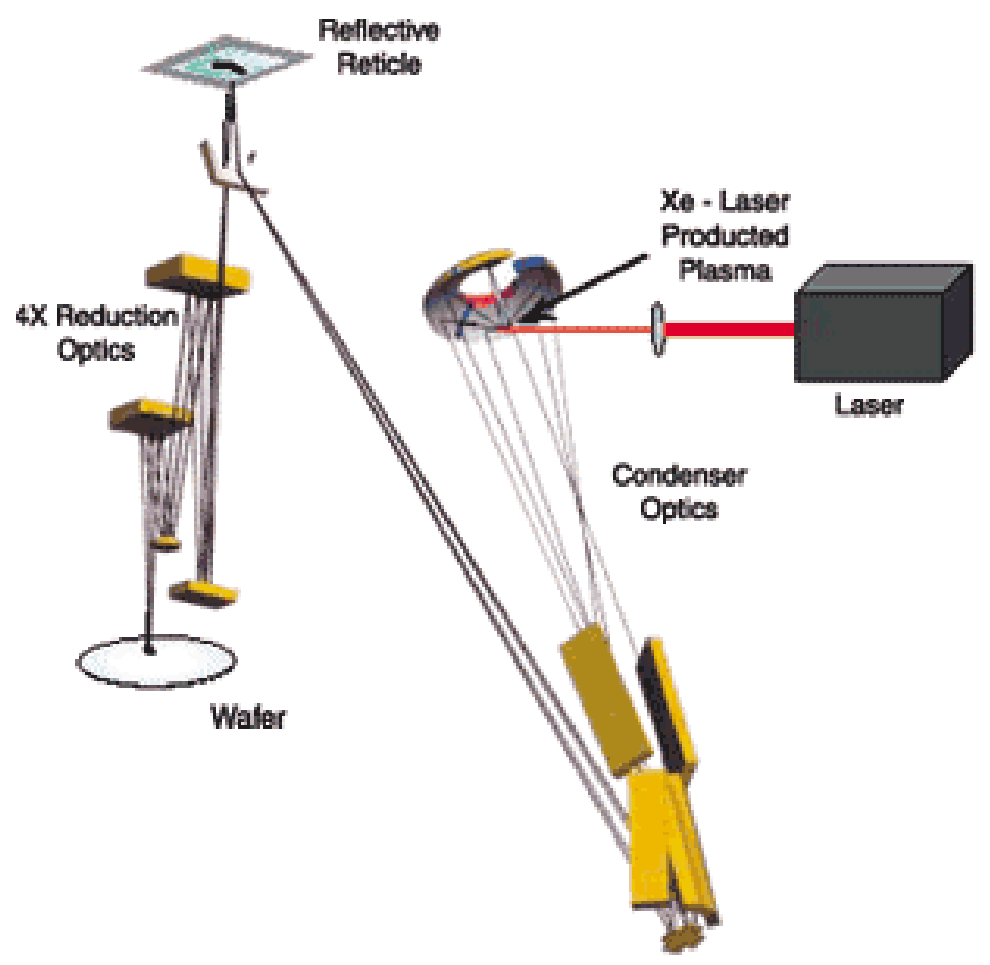

Figure 1.2 Schematic diagram of the EUV lithography system. ${ }^{4}$

In principle, EUV is a logical extension of optical lithography to very short wavelengths. A schematic diagram of a EUV lithography tool is shown in Fig. 1.2. A laser-induced Xe plasma or synchrotrons radiation is used to generate 13-nm-wavelength 
EUV, which is collected by condenser optics and focused as a narrow arc of illumination onto a reflective reticle. The reticle is imaged onto the wafer using $4 \times$ reduction optics realized by four mirrors coated with multilayers of $\mathrm{Mo} / \mathrm{Si} .{ }^{4}$ It has been demonstrated that all the components of EUV technology can be integrated into a fully functional prototype EUV exposure tool, which can pattern 70nm features. ${ }^{5,6}$

Currently, the EUV source and multiplayer-coating masks are the most challenging hurdles facing EUV lithography. A high power source that can support an exposure tool throughput of sixty $300 \mathrm{~mm}$ wafers per hour is required to assure EUV for massive IC production. Since even very small $(30 \AA)$ defects in the multilayers can print unwanted features on the wafer, ${ }^{2}$ another serious issue for EUV lithography is in creating multilayer coating masks with no defects.

\subsubsection{X-ray lithography}

X-ray lithography has been under development since the early 1980s, reaching an advanced level of performance once the $1 \times$ mask-making had been solved and the synchrotron radiation $\mathrm{x}$-ray source adopted. The main technical limitation of $\mathrm{x}$-ray lithography is mask making, as the $1 \times$ reticle must be manufactured, compared to $4 \times$ or $5 \mathrm{x}$ as for other systems. This places $\mathrm{x}$-ray lithography used mainly on the pattern generation machines, which are not yet capable of delivering the throughput and accuracy acquired for next generation lithography tools. ${ }^{7}$

Research has demonstrated that devices can be scaled down to sub-20 $\mathrm{nm}$ and smaller, ${ }^{8-10}$ therefore much shorter wavelength radiation need to be considered for patterning such small features. 
The wavelength for charge particles, such as electrons and ions, can be calculated using the following formula.

$$
\lambda=\frac{h}{\sqrt{m E / 2}}
$$

where $h$ is Plank constant, $m$ is the mass, and $E$ is the energy of the charge particle. For $100 \mathrm{keV}$ electron beam, the wavelength is about $4 \times 10^{-3} \mathrm{~nm}$, while for $100 \mathrm{keV}$ helium ion beam, the wavelength is about $5 \times 10^{-5} \mathrm{~nm}$. The higher the mass and the energy of the particle, the shorter is the wavelength. Instead of being diffraction limited, the electron beam or ion beam lithography tool are limited by the aberration of the optical design. Theoretically, they can be used as lithography tools at $30 \mathrm{~nm}$ node and beyond.

\subsubsection{Electron beam lithography}

There are two major electron projection lithography schemes: the scattering with angular limitation projection electron-beam lithography (SCALPEL) approach started at Bell Laboratories ${ }^{11}$ and the projection reduction exposure with variable axis immersion lenses (PREVAIL) which is pursuing by IBM in cooperation with Nikon. ${ }^{12,13}$

The SCALPEL approach combines the high resolution and wide process latitude inherent in electron beam lithography with the throughput of a parallel projection system and avoids the problems of stencil masks. As shown in Fig 1.3(a), in the SCALPEL system, a mask consisting of a low atomic number membrane and a high atomic number pattern layer is uniformly illuminated with high energy $(100 \mathrm{keV})$ electrons. The entire mask structure is essentially transparent to the electron beam and little of its energy is deposited there. The portions of the beam that pass through the high atomic number pattern layer are scattered through angles of a few miliradians. An aperture in the back 
focal plane of the electrons produces a high contrast image at the plane of the semiconductor wafer. $^{11}$
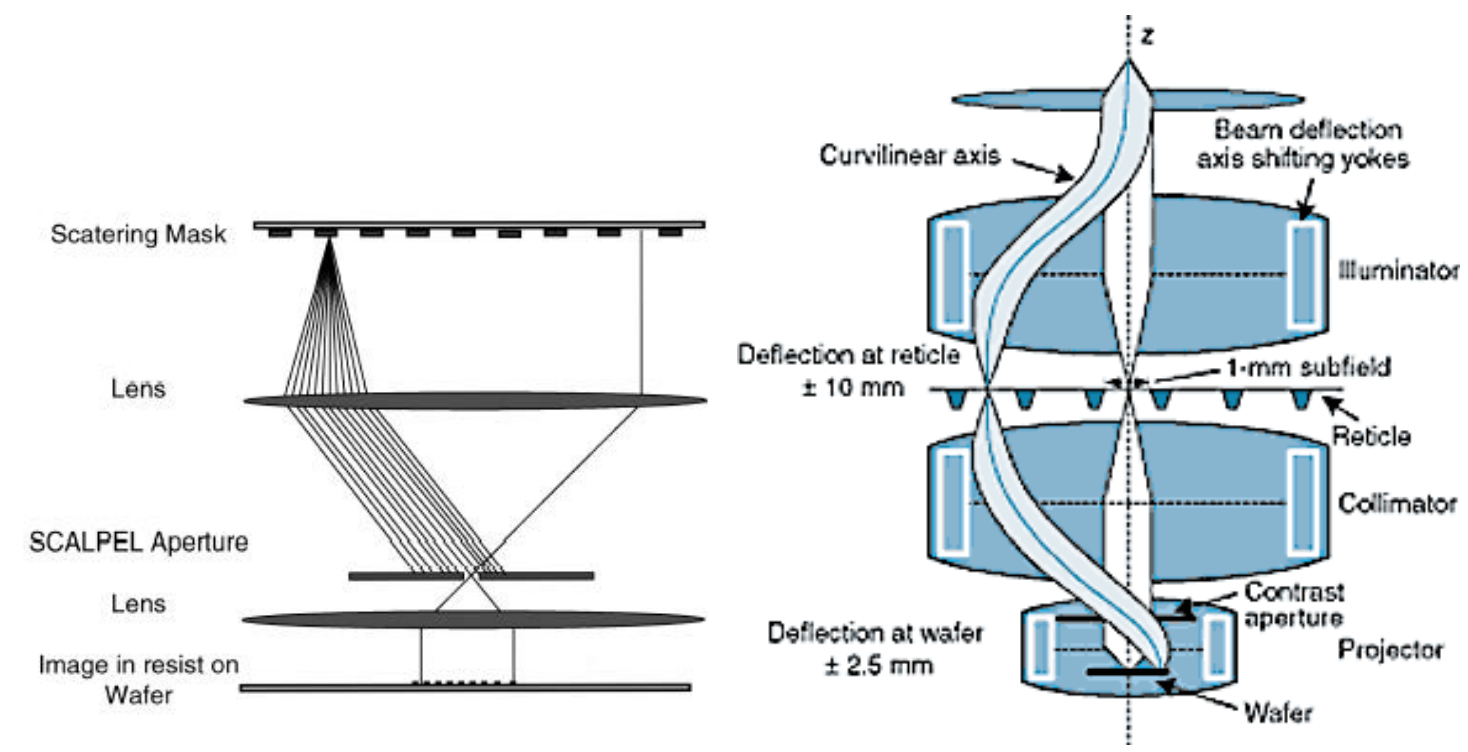

Figure 1.3 (a) Schematic diagram of the SCALPEL step-and-scan system ${ }^{11}$, (b) Schematic diagram of PREVAIL imaging concept of curvilinear variable-axis lenses. ${ }^{12}$

Figure 1.3(b) shows the basic PREVAIL imaging concept. The illuminator is a magnetic lens system providing illumination for each subfield pattern by imaging a $1 \times 1$ $\mathrm{mm}$ shaped beam onto the reticle. Collimator and projector lenses are forming an antisymmetric telecentric doublet known to inherently have a minimum of geometric aberrations. The curved beam path schematically illustrates illumination and imaging of a subfield at the edge of the optical field of view. The PREVAIL technique permits shifting of the electron optical axis along a predetermined curvature, while simultaneously deflecting the electron beam to precisely follow the curvilinear variable axis. ${ }^{12}$

The supreme challenge facing all e-beam lithography approaches has been and still is throughput in wafers per hour. Throughput in electron-beam lithography (EBL) is directly proportional to the total beam current delivered to expose a resist-coated wafer. 
High beam current produces increased blur, which limits lithography resolution. For a single beam, it will be increasingly difficult to obtain throughput due to electron-electron interaction. Multiple beam system is one of the solutions to overcome this problem.

\subsubsection{Ion beam lithography}

Efforts have been devoted to developing ion projection lithography (IPL) in Europe and Unite States. Figure 1.4(a) shows that IPL uses the same principles as optical steppers with mask patters being printed to resist coated wafer substrates. Hydrogen or Helium ions are extracted from an ion source. Multi-electrode electrostatic ion optics is implemented to generate a broad ion beam illuminating the reticle and to project the image of the stencil mask patterns to the wafer substrate. ${ }^{14}$ Lines with $50 \mathrm{~nm}$ in width can be printed with an exposure dose of $0.3 \mu \mathrm{C} / \mathrm{cm}^{2}$ of $75 \mathrm{keV} \mathrm{He}^{+}$ions into standard 300 nm thick DUV resist (Shipley UV II HS). ${ }^{15}$

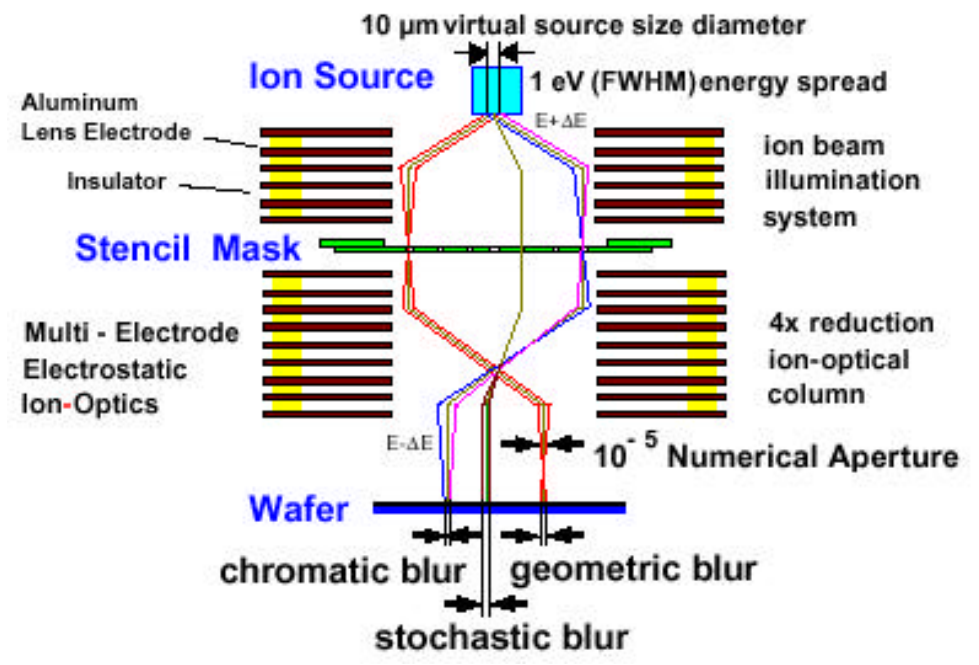

(a) 


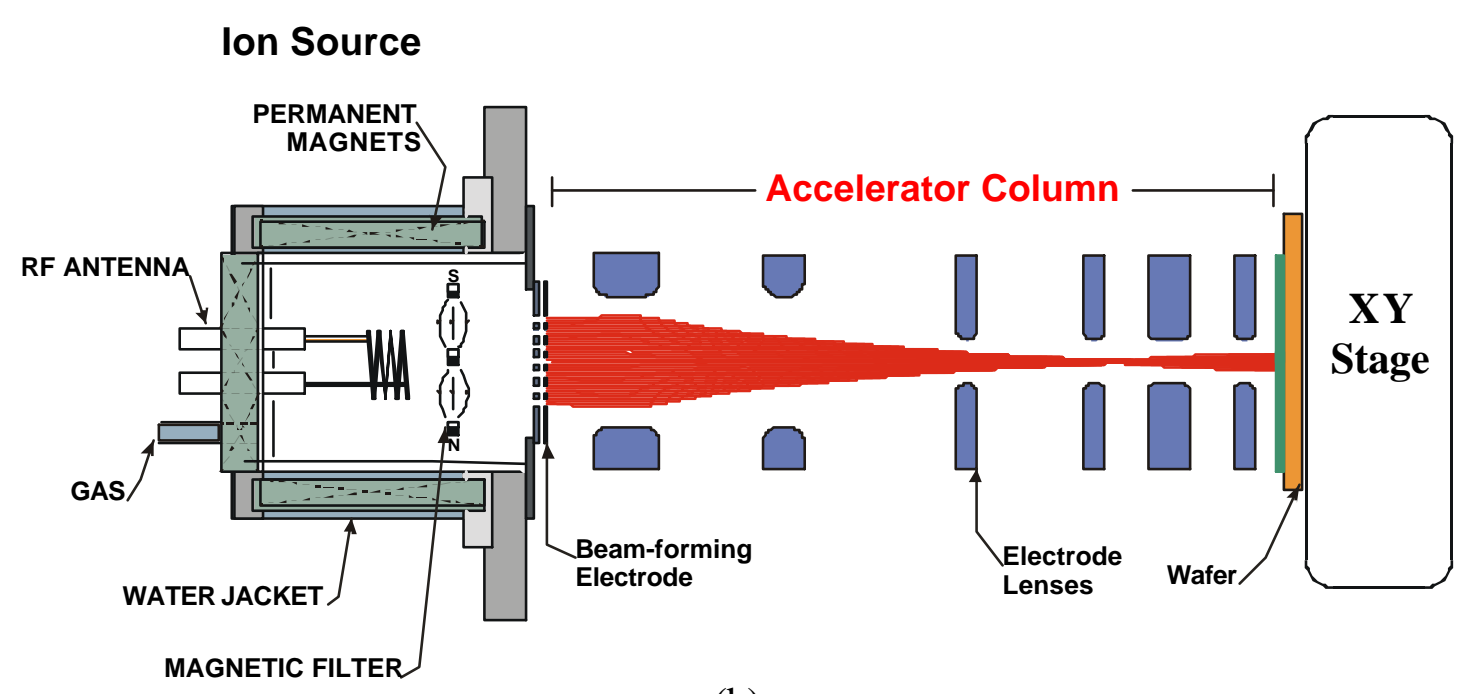

(b)

Figure 1.4 (a) European MEDEA Ion Projection lithography. ${ }^{14}$ (b) Schematic of Ion Projection Lithography in LBNL. ${ }^{16}$

The thickness of a stencil mask is only of a few microns, therefore it is very fragile and hard to handle. Fabrication of a stencil mask is very costly, similar to the mask involved in electron beam lithography. The Maskless Micro-ion beam Reduction Lithography (MMRL) ${ }^{16}$ scheme shown in Figure 1.4(b) is to completely eliminate the first stage of the conventional IPL system that contains the complicated beam optics design in front of the stencil mask and the mask itself. The main components of the MMRL system consist of a multi-cusp plasma generator, a multi-beamlet extraction system, and an accelerator column for beam reduction. A universal pattern generator, with an array of multi-beamlet apertures, which can be individually switched on or off by biasing each aperture in the second electrode with respect to the first, is used to generate any desired pattern to be projected onto the wafer substrate. Feature size as small as $166 \mathrm{~nm}$ has been demonstrated. $^{17}$ 
Ions are much heavier than electrons. Therefore, the wavelength of ions is two orders of magnitude shorter than electrons at the same energy. Ion beam resolution higher than electron beam may be achievable. The actual beam size achieved by an ion projector is governed by ion source parameters (virtual source size, energy spread of the extracted ions) and errors of the electrostatic lens system.

Another advantage of using ions over electrons is that ions experience much reduced backscattering and proximity effect. Resists are more sensitive to ions too, which results in potentially higher throughput. However, it may bring in shot noise problem in resist exposure. ${ }^{18}$

\subsection{Maskless, resistless ion beam lithography}

\subsubsection{Motivation}

As mentioned above, significant challenges exist today for each of these techniques: issues with complicated mask technology for EUV, X-ray and projection ion-beam; issues with photoresist material and proximity effect for e-beam lithography. A lot of progress has been made on non-conventional lithography techniques such as nanoimprint, but none can do without masks. ${ }^{19-22}$

This thesis is to develop a maskless, resistless ion beam lithography process. By eliminating masks and resist, not only all the related issues will be eliminated, but also the processes will be much cheaper and much simpler. Such technology would have enormous cost savings for the semiconductor industry by eliminating technology efforts for mask developments, defect detection, and defect correction. Other advantages of a maskless system are the flexibility for the implementation of new design quickly, 
producing chips with difference functions on the same wafer, which would save the industry millions of dollars by allowing rapid implementations of performance and yield improvements.

\subsubsection{Maskless, resistless ion beam lithography processes}

Focused oxygen ions can be used to form a thin silicon dioxide layer on the silicon surface, which serves as a hard mask in reactive ion etching process when patterning the gate area. Focused boron or phosphorus ion beams can be directly implanted to form shallow junction. Fig. 1.5 shows a plane view of a SOI MOSFET. Both source/drain area and gate can be patterned using focused oxygen ion beam, and source/drain area can be selectively doped with FIB. There is no mask and resist involved in the whole process.

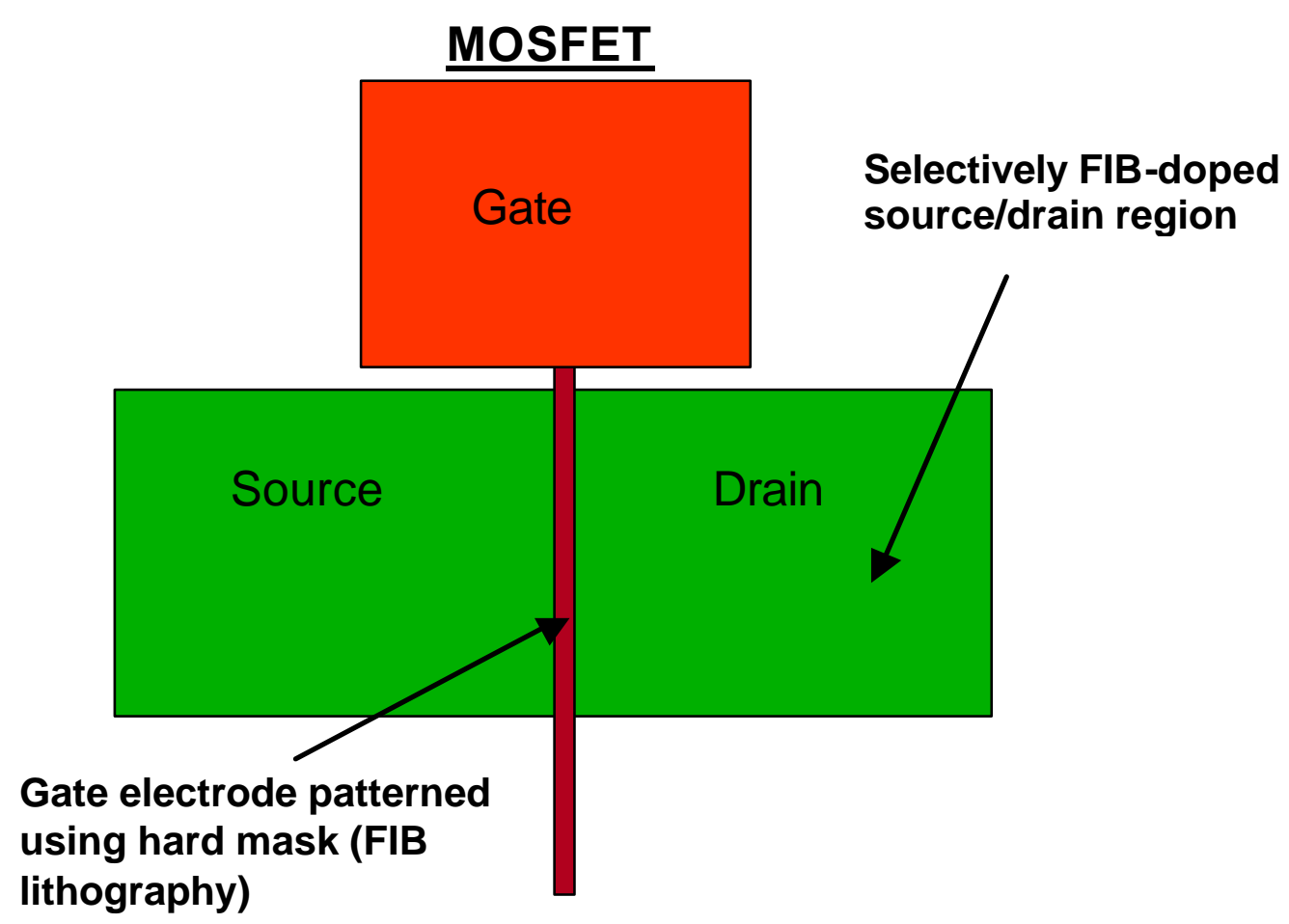

Figure 1.5 MOSFET fabrication using maskless, resistless ion beam lithography. 
The development of a maskless, resistless ion beam lithography system has been carried out in Lawrence Berkeley National Laboratory. Fig. 1.6 shows the dedicated test stand in a clean room facility. The basic components of such a system are the following: (1) a multicusp plasma ion source, (2) a focused ion beam column, (3) a vacuum chamber with a typical base pressure of mid $10^{-7}$ Torr, (4) a vibration isolation platform, (5) a multi-axis stage for precise substrate positioning and scanning, (6) a load lock for sample handling, (7) power supplies for system operation, and (8) a gas delivery system.

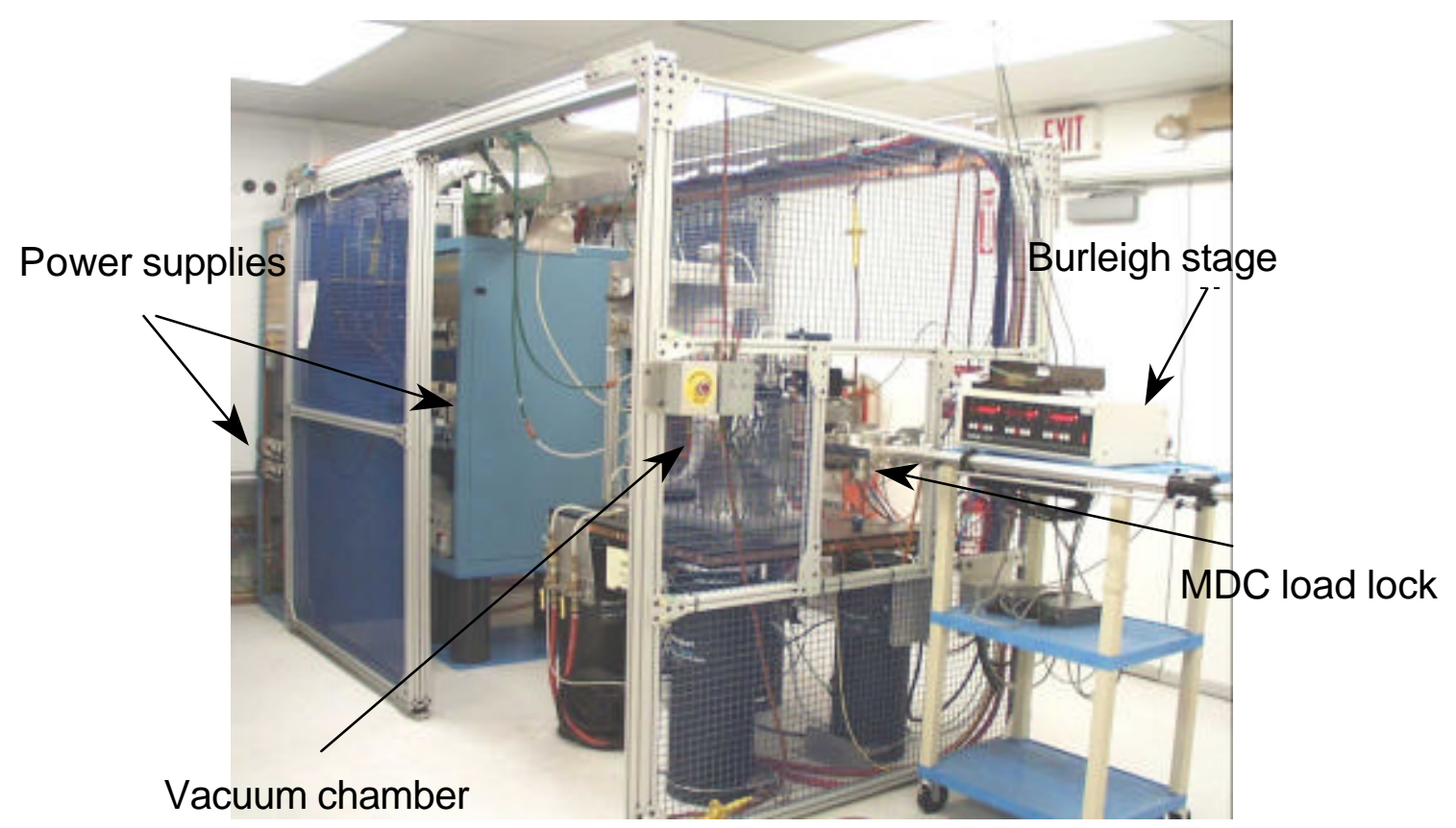

Figure 1.6 Picture of dedicated test stand of maskless, resistless ion beam lithography system. 


\subsubsection{Ion source requirement}

High-resolution focused ion beams (FIB) have been applied in a number of technologically important ways: maskless implantation into semiconducting materials; lithographic mask repair for visible light and $\mathrm{x}$ rays; micromachining to create micrometer structures; deposition of materials from the vapor phase by ion-induced decomposition; modification of integrated circuits; failure analysis as part of the integrated circuit manufacturing process; and surface analysis. ${ }^{23-24}$ In a conventional FIB system, a liquid-metal ion source (LMIS) is used to deliver current in the range of a few hundred pA into a spot size of approximately $50 \mathrm{~nm} \cdot{ }^{23}$ The disadvantage of the LMIS is that it is limited to producing only certain metallic elements such as gallium. In recent years, a new type of source, the gas field ionization source (GFIS) has been developed for operation in a gaseous phase. ${ }^{25}$ The GFIS is capable of generating high-brightness ion beams of hydrogen, helium or neon that can be focused down to about $10 \mathrm{~nm}$ spot size. However, there are some disadvantages of the GFIS. A sophisticated cryogenic system is needed for maintaining a gas reservoir at the "super-tip". Also, the extractable current typically decreases with increasing ion mass.

In 1970s, Seliger et al ${ }^{26-28}$ first attempted to use ion beams in transistor fabrication. However, in order to achieve resistless lithography, the ion source has to be able to produce variable ion species, such as oxygen, boron, and phosphorus. Multicusp plasma ion source can generate various ion species, which makes resistless processes possible. Chapter 2 will discuss the principle of a multicusp plasma ion source, summarize the characteristics, such as the energy spread of the beam extracted from the source, the current density of the beam as a function of discharge power, extraction voltage, etc. The 
production of oxygen, boron, and phosphorus ion beam and the direct patterning processes will be presented in Chapter 5.

\subsubsection{Throughput requirement}

Throughput has historically been a prohibitive issue for FIB's application to lithographic processes in semiconductor manufacturing. Clearly, in order for this technology to be viable for high-volume manufacturing, significant improvements in beam brightness and current density must be attained to achieve higher process throughput.

Chapter 3 will present the spectral source brightness measurement, and then discuss the improvement of the beam brightness. The beam brightness of a multicusp plasma ion source can be substantially improved by optimizing the source configuration and extractor geometry.

All-electrostatic lenses are often used to focus massive particles such as ions because round magnetic lenses have rather weak focusing properties for ion. Chapter 4 will present the design of a compact accelerator column for focused $\mathrm{O}_{2}^{+}$beam formation. Ion transport code IGUN $^{29}, 30$ and charge particle optics design software ${ }^{31}$ provided by Munro's Electron Beam Software Ltd. are used to analyze and optimize the performance of such an accelerator column. Beam spot size results will be presented, and also confirmed by experimental measurements.

The use of a multibeam approach can also result in improved throughput while maintaining pattern accuracy and quality. Similar approach has also been used in maskless electron beam lithography for throughput enhancement. ${ }^{32-35}$ Fig 1.7 shows the 
basic concept of the arrayed microcolumn approach. An array of miniaturized electron beam column is used to generate patterns in parallel to achieve high throughput. Patterns can be written in either raster- or vector-scan mode with the beam scanning over only a narrow stripe, with a laser-controlled stage moving continuously in the orthogonal direction to build up the complete chip pattern. The current in each beam can be considerably reduced and at the same time, a high total current can be maintained at the wafer surface.
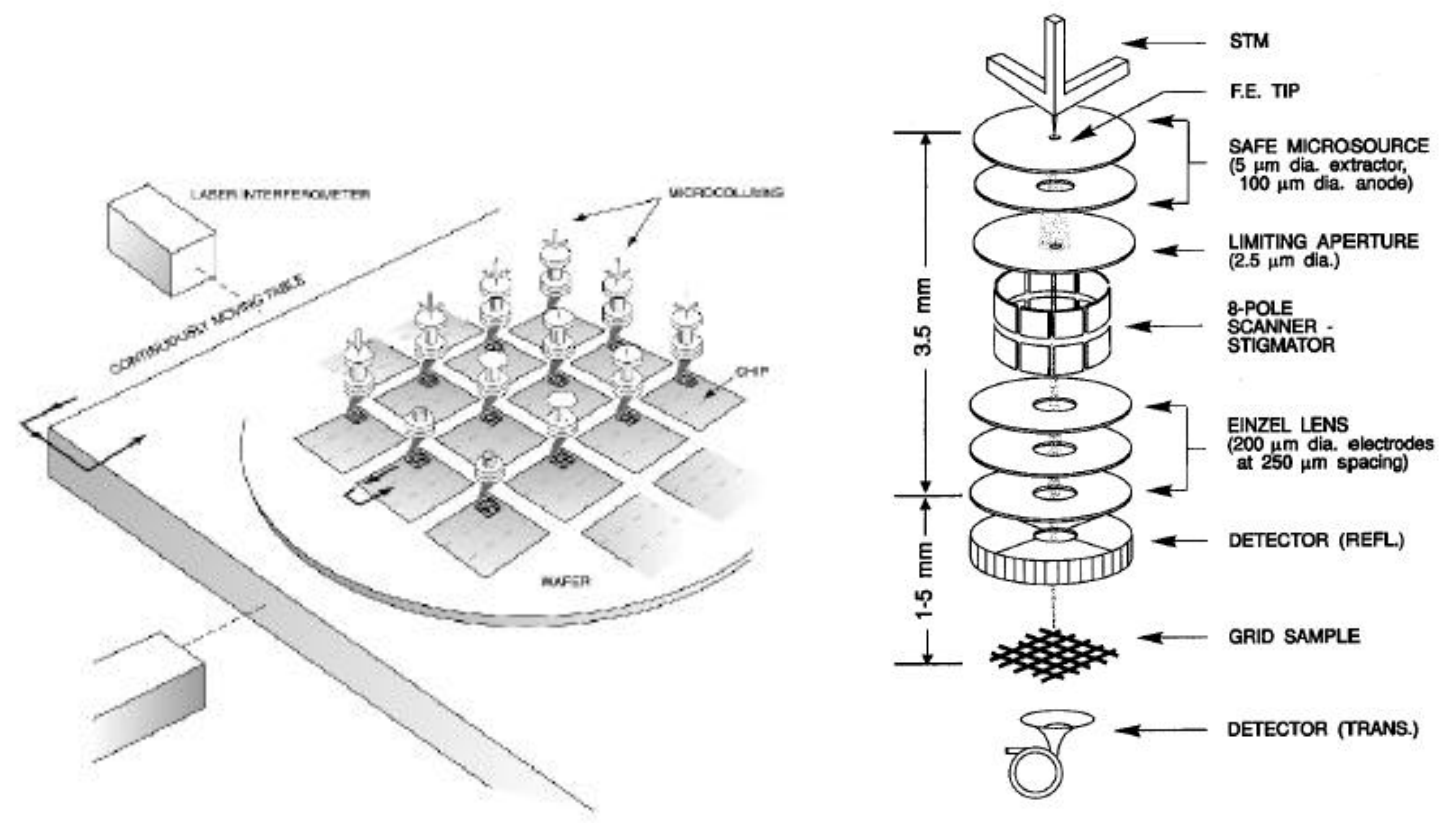

Figure 1.7 (a) Schematic diagram of the arrayed microcolumn e-beam lithography concept. (b) Schematic diagram of miniature e-beam column. ${ }^{33}$

The multibeam focused ion beam lithography system is very similar to the arrayed microcolumn e-beam system shown above, the difference is that only one multicusp plasma source is used to generate uniform, larger area plasma. By applying different bias on a stack of electrodes with multiple apertures which act as extractors, the beam can be 
turned on and off; therefore no mask is needed to generate different patterns. The detailed discussion of the multi-beam system will be presented in Chapter 6 .

\section{References:}

1 G. Moore, "Progress in digital integrated electronics", in Proc. IEEE Int. Electron Devices Meeting, Dig. Tech. Papers, pp11, 1995.

${ }^{2}$ H. R. Harriott, 'Limits of Lithography", Proceeding of the IEEE, vol. 89, No. 3, pp366, March 2001.

${ }^{3}$ Semiconductor Industry Association (SIA), The International Technology Roadmap for Semiconductors: 2001.

4 C. W. Gwyn, R. Stulen, D. Sweeney, and D. Attwood, "Extreme ultraviolet lithography”, J. Vac. Sci. Technol. B 16(6), p3142, 1998.

5 C. Gwyn, "EUV lithography update", $O E$ magazine, Publication of SPIE-The international society for optical engineering, June 2002.

${ }^{6}$ H. N. Chapman, A. K. Ray-Chaudhuri, D. A. Tichenor, W. C. Replogle, R. H. Stulen, G. D. Kubiak, P. D. Rockett, L. E. Klebanoff, D. O'Connell, A. H. Leung, K. L. Jefferson, J. B. Wronosky, J. S. Taylor, L. C. Hale, K. Blaedel, E. A. Spiller, G. E. Sommargren, J. A. Folta, D. W. Sweeney, E. M. Gullidson, P. Naulleau, K. A. Goldberg, J. Bokor, D. T. Attwood, U. Mickan, R. Hanzen, E. Panning, P. -Y. Yan, C. W. Gwyn, and S. H. Lee, "First lithographic results from the extreme ultraviolet Engineering Test Stand”, J. Vac. Sci. Technol. B 19(6), p2389, Nov/Dec 2001.

7 S. Hector, "Status and Future of X-ray Lithography", Microelectronic Engineering, 41/42, pp25-30. 1998. 
${ }^{8}$ X. Huang, W.-C. Lee, C. Kuo, D. Hisamoto, L. Chang, J. Kedzierski, E. Anderson, H. Takeuchi, Y.-K. Choi, K. Asano, V. Subramanian, T.-J. King, J. Bokor and C. Hu, "Sub50 nm p-channel FinFET," IEEE Transactions on Electron Devices, Vol. 48, No. 5, pp. 880-886, 2001.

9 J. Kedzierski, P. Xuan, E. H. Anderson, J. Bokor, T.-J. King and C. Hu, "Complementary silicide source/drain thin-body MOSFETs for the 20nm gate length regime," International Electron Devices Meeting Technical Digest, pp. 57-60, 2000.

${ }^{10}$ Y.-K. Choi, N. Lindert, P. Xuan, S. Tang, D. Ha, E. Anderson, T.-J. King, J. Bokor and C. Hu, "Sub-20nm CMOS FinFET technologies," International Electron Devices Meeting Technical Digest, pp. 421-424, 2001.

11 L. R. Harriott, "Scattering with angular limitation projection electron beam lithography for suboptical lithography”, J. Vac. Sci. Technol. B 15(6), p2130(1997).

12 C. Pfeiffer, R. S. Dhaliwal, S. D. Golladay, S. K. Doran, M. S. Gordon, T. R. Groves, R. A. Kendall, J. E. Lieberman, P. F. Petric, D. J. Pinckney, R. J. Quickle, C. F. Robinson, J. D. Rockrohr, J. J. Senesi, W. Stickel, E. V. Tressler, A. Tanimoto, T. Yamaguchi, K. Okamoto, K. Suzuki, T. Okino, S. Kawata, K. Morita, S. C. Suziki, H. Shimizu, S. Kojima, G. Varnell, W. T. Novak, D. P. Stumbo, and M. Sogard, "Projection reduction exposure with variable axis immersion lenses: Next generation lithography", $J$. Vac. Sci. Technol. B 17(6), p2840(1999).

13 H.C. Pfeiffer, R.S. Dhaliwal, S.D. Golladay, S.K. Doran, M.S. Gordon, R.A. Kendall, J.E. Lieberman, D.J. Pinckney, R.J. Quickle, C.F. Robinson, J.D. Rockrohr, W. Stickel, E.V. Tressler, A. Tanimoto, T. Yamaguchi, K. Okamoto, K. Suzuki, T. Miura, T. Okino, S. Kawata, K. Morita, S.C. Suzuki, H. Shimizu, S. Kojima, G. Varnell, W.T. Novak, and 
M. Sogard, 'PREVAIL e-beam stepper alpha tool", Microelectronic Engineering 57-58, p163(2001).

14 R. Kaesmaier and H. Loschner, "Overview of the Ion Projection Lithography European MEDEA and Internation Program", SPIE Conference on Microlithography, Santa Clara, California, USA, Feb. 28-March 1, 2000.

15 W. H. Bruenger, M. Torkler, K. N. Leung, Y. Lee, M. D. Williams, H. Loeschner, G. Stengl, W. Fallmann, F. Paschke, G. Stangl, I. W. Rangelow, and P. Hudek, "Resolution Improvement of Ion Projector with a Low Energy Spread Multicusp Ion Source", Microelectronic Engineering, 46, 477(1999).

${ }^{16}$ V. V. Ngo, W. Barletta, R. Gough, Y. Lee, K. N. Leung, N. Zahir, and D. Patterson, "Maskless micro-ion-beam reduction lithography", J. Vac. Sci. Technol. B 17(6), 2783 (1999).

17 V. V. Ngo, B. Akker, K. N. Leung, I. Noh, and S. Wilde, "Demonstrations of Electronic Pattern Switching and 10x pattern demagnification in a maskless micro-ionbeam reduction lithography system", $46^{\text {th }}$ International Conference on Electron, Ion, and Photon Beam Technology and Nanofabrication, Anaheim, CA, May28-31, 2002.

18 J. Melngailis, A. A. Mondelli, I. L. Berry III, and R. Mohondro, "A review of ion projection lithography”, J. Vac. Sci. Technol. B 16, 927(1998).

19 S. Y. Chou, P. R. Krauss, and P. J. Renstrom, "Nanoimprint lithography", J. Vac. Sci. Technol. B. 14, 4129(1996).

20 S. J. Schablitsky, Z. Yu, and S. Y. Chou, "Application of nanoimprint lithography in annoscale GaAs MSM photodetectors", Technical Digest Summaries of papers presented 
at the Conference on Lasers and Electro-Optics, Baltimore, MD, USA, May 1999. pp578.

${ }^{21}$ I. Martini, M. Kamp, F. Fischer, L. Worschech, J. Koeth, and A. Forchel, 'Fabrication of quantum point contacts and quantum dots by imprint lithography", Microelectronic Engineering, 57-58, 397(2001).

22 W. Zhang, and S. Y. Chou, "Multilevel nanoimprint lithography with submicron alignment over 4 in. Si wafers", Applied Physics Letters, 79, 845(2001).

${ }^{23}$ J. Orloff, "High-resolution focused ion beams", Rev. Sci. Instrum, 64(5), pp1105, 1993.

24 J. Melngailis, "Focused ion beam technology and applications", J. Vac. Sci. Technol. B5, pp469, 1987.

25 Ch. Wilbertz, Th. Miller, and S. Kalbitzer, "Recent progress in Gas Field Ion Source technology", Procedding of SPIE, vol. 2194, 407(1994).

26 R. L.Seliger, and W. P. Fleming, "Focused ion beams in microfabrication", J. Appl. Phys. 45, p1416, 1974.

${ }^{27}$ R. L. Seliger, R. L. Kubena, R. D. Olney, J. W. Ward, V. Wang, "High-resolution, ionbeam processes for microstructure fabrication”, J. Vac. Sci. Technol. 16(6), p1610, 1979.

28 D. B. Rensch, R. L. Seliger, G. Csanky, R. D. Olney, and H. L. Stover, 'Ion-beam lithography for IC fabrication with submicrometer features", J. Vac. Sci. Technol. 16(6), p1897, 1979.

${ }^{29}$ R. Becker, and W. B. Herrmannsfeldt, 'IGUN-a program for the simulation of positive ion extraction including magnetic fields", Rev. Sci. Instrum., 4, pp. 2756-2758, 1992.

${ }^{30}$ R. Becker, Numerical simulation of ion-beam formation", Rev. Sci. Instrum. 67, pp. 1132-1137, 1996. 
${ }^{31}$ MEBS Ltd., England. 'Munro’s Electron Beam Software User Manual”, 1997.

32 T. H. P. Chang, M. Mankos, K. Y. Lee, and L. P. Muray, "Multiple electron-beam lithography”, Microelectronic Engineering 57-58, pp117, 2001.

33 T. H. P.Chang, M.G.R. Thomson, E. Kratschmer, H. S. Kim, M. L. Yu, K. Y. Lee, S. A. Rishton, and B. W. Hussey, "Electron-beam microcolumns for lithography and related applications", J.Vac.Sci.Technol.B 14(6), pp3774, 1996.

34 L.P.Muray, J.P.Spallas, C.Stebler, K.Lee, M.Mankos, Y.Hsu, M.Gmur, and T. H. P. Chang, "Advances in arrayed microcolumn lithography", J. Vac. Sci. Technol.B 18(6), pp3099, 2000.

${ }^{35}$ M.Mankos, S.Coyle, A.Fernandez, A.Sagle, W.Owens, J. Sullivan, and T. H. P. Chang, "Basic constraints for a multibeam lithography column", J. Vac. Sci. Technol. B 19(2), pp467, 2001. 


\section{Chapter 2}

\section{Multicusp Plasma Ion source for MRIBL}

\subsection{Overview of FIB}

Focused ion beams (FIBs) have been used extensively in the semiconductor industry for many applications such as micromachining, mask repair, circuit modification and failure analysis. ${ }^{1}$ As the lateral dimensions of semiconductor devices are scaled down, next generation lithography (NGL) technology will replace optical lithography integrated-circuit manufacture. Among four major alternative NGL approaches, ion beam lithography is the only one which can provide both maskless and resistless patterning. As such, it can potentially make nano-fabrication much simpler. For this to happen, the ion source must produce a variety of ion species.

Figure 2.1 is a plot of a conventional FIB system ${ }^{4}$, which consists of an ion source, a condenser lens, a $\mathrm{E} \times \mathrm{B}$ filter to select certain ion species, an objective lens to focus the beam, and a sample holder. In such a system, a liquid-metal ion source (LMIS) is usually employed, which is limited to producing only certain metallic elements such as gallium. 


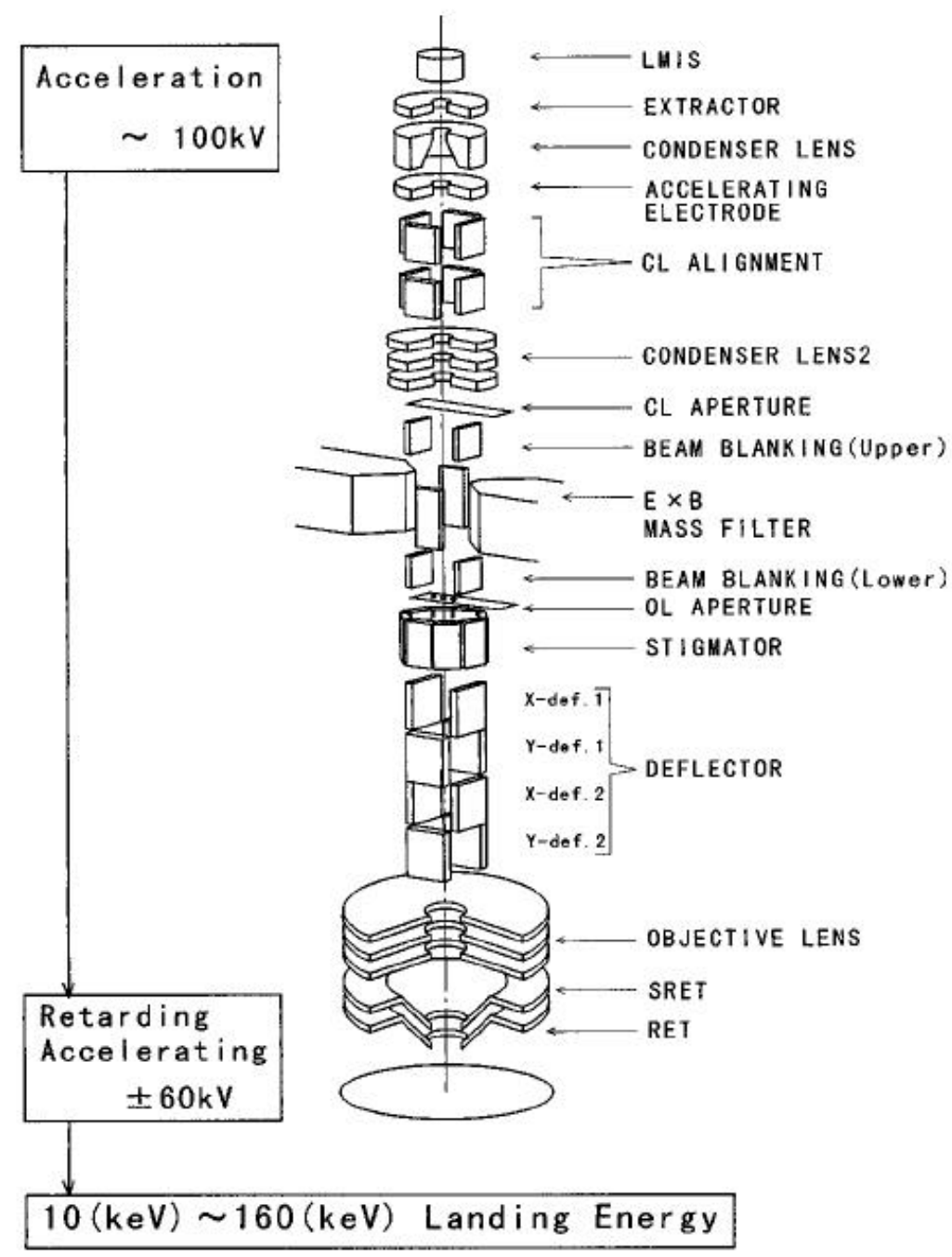

Figure 2.1 A conventional FIB system: Source (LMIS), condenser lens, EXB filter, objective lens, sample holder. ${ }^{4}$

There has been some research on direct deposition of insulator or metallic material using FIB system. Since LMIS is limited to Ga ion production, a certain precursor gas is needed. The deposition process ${ }^{5}$ is illustrated in Figure 2.2: first, the precursor gases are sprayed on the surface by a fine needle ("nozzle"), where they are adsorbed; second, the incoming ion beam decomposes the adsorbed precursor gases; then, the volatile reaction products are desorbed from the surface and are removed through the vacuum system, while the desired reaction products $\left(\mathrm{W}\right.$ or $\mathrm{SiO}_{2}$ ) remain fixed on the surface as a thin 
film. Using this approach, researchers have successfully deposit $\mathrm{SiO}_{2}{ }^{5}$ and $\mathrm{Au}^{6}, \mathrm{~W}, \mathrm{Pt}$, $\mathrm{Cu}$ onto $\mathrm{Si}$ film, ${ }^{7-10} \mathrm{Si}$ in GaAs film ${ }^{11}$. However, the deposited material is not fully pure because organic contaminants as well as Ga atoms (from the ion beam) are inevitably included in the deposited film. ${ }^{5}$ Therefore, conventional FIB system with LMIS is not suitable for resistless lithography. Ion source that can generate variable elements needs to be investigated.

Another disadvantage of conventional FIB lithography system is low throughput. Although multiple beams can enhance the speed of exposure process, it is not practical to incorporate thousands of LMIS's together into one system. Fabrication, operation, and maintenance of such a huge system may be a nightmare. The current uniformity of each single LMIS will also increase the burden of control circuit. In a word, a uniform, large area plasma ion source is one promising solution.

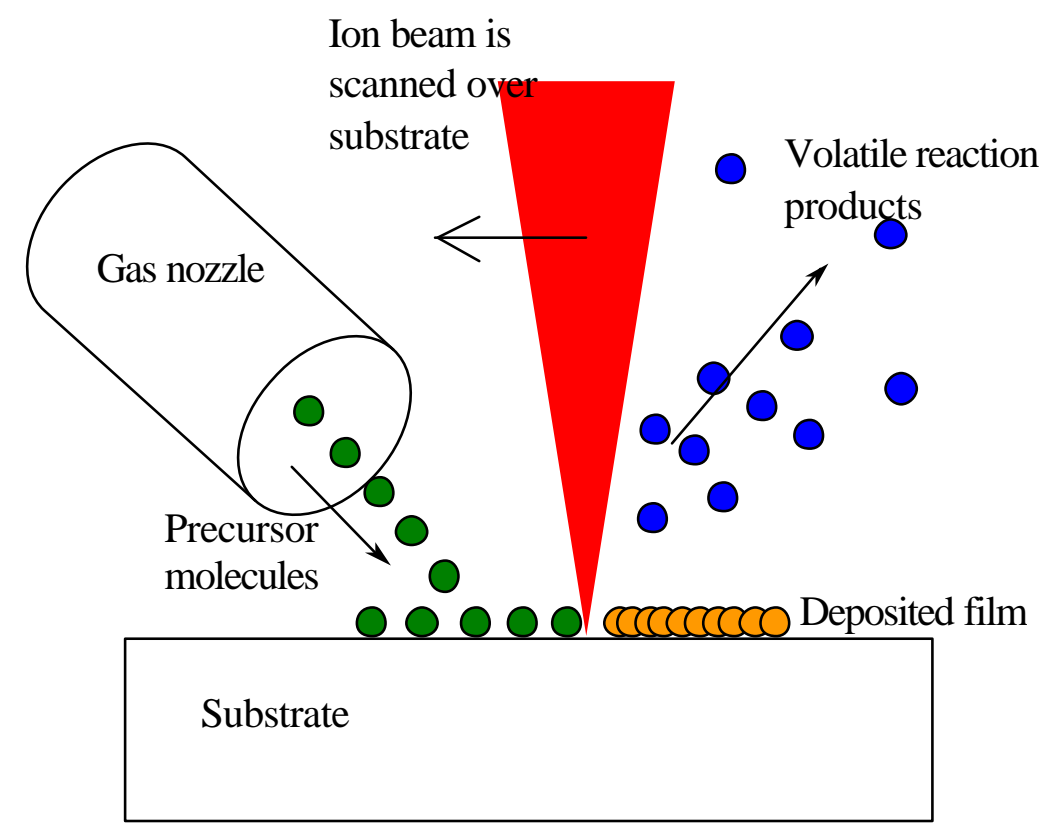

Figure 2.2 Direct deposition with precursor gas using FIB system. ${ }^{5}$ 


\subsection{Multicusp Plasma Ion Source}

Plasma sources, in particular multicusp generators, have been used for many applications, such as in neutral beam injectors for fusion devices, particle accelerators, ion implantation systems, neutron tubes for oil well logging and proton therapy machines. $^{12,13}$

\subsubsection{Principle of multicusp plasma source}

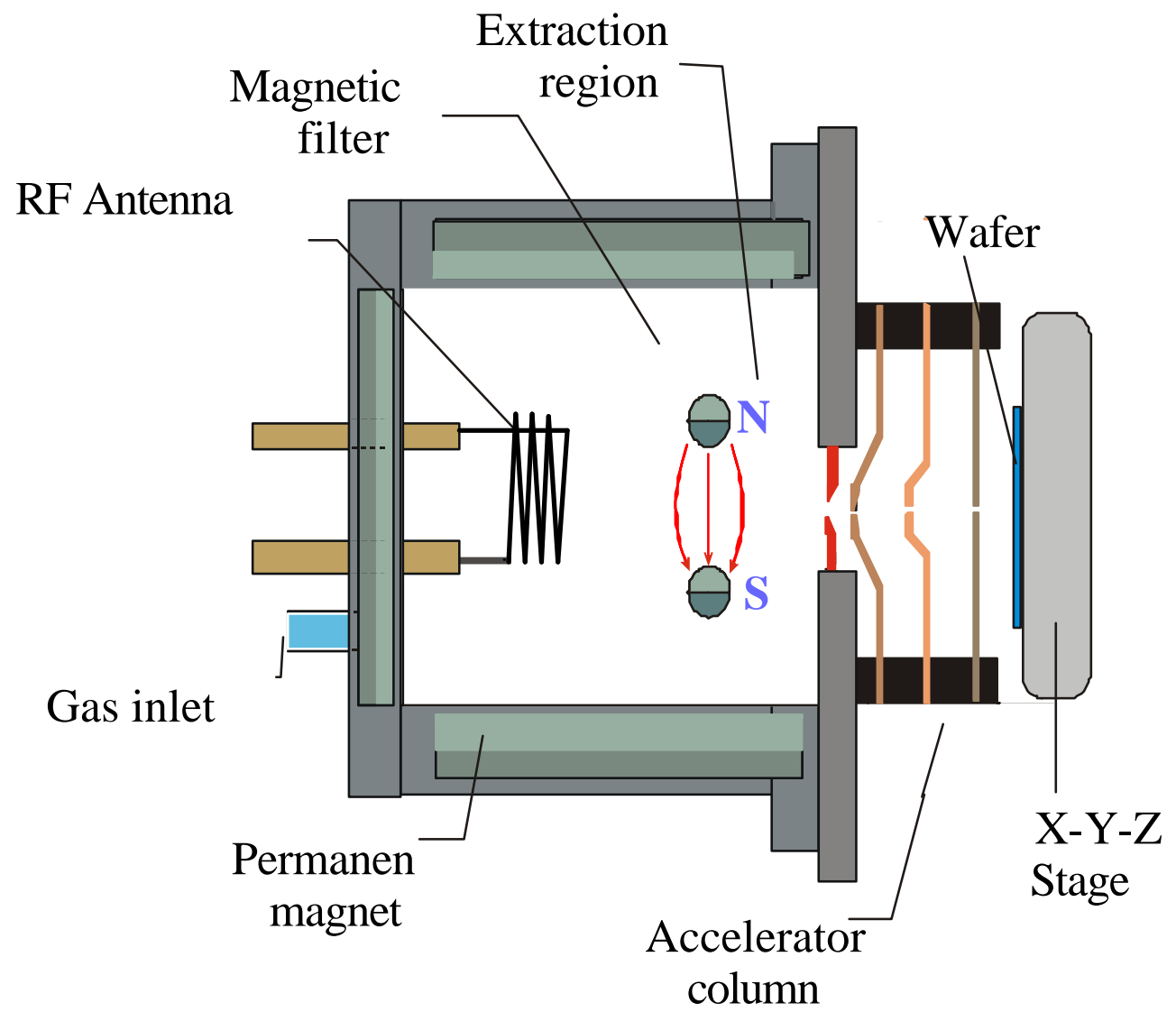

Figure 2.3 Schematic of a RF-driven multicusp plasma source

Figure 2.3 shows a schematic diagram of a RF driven multicusp ion source. The source is surrounded with columns of permanent magnets. The surface magnetic field generated 
by rows of permanent magnets, typically of samarium-cobalt, can confine the primary ionizing electrons very efficiently.

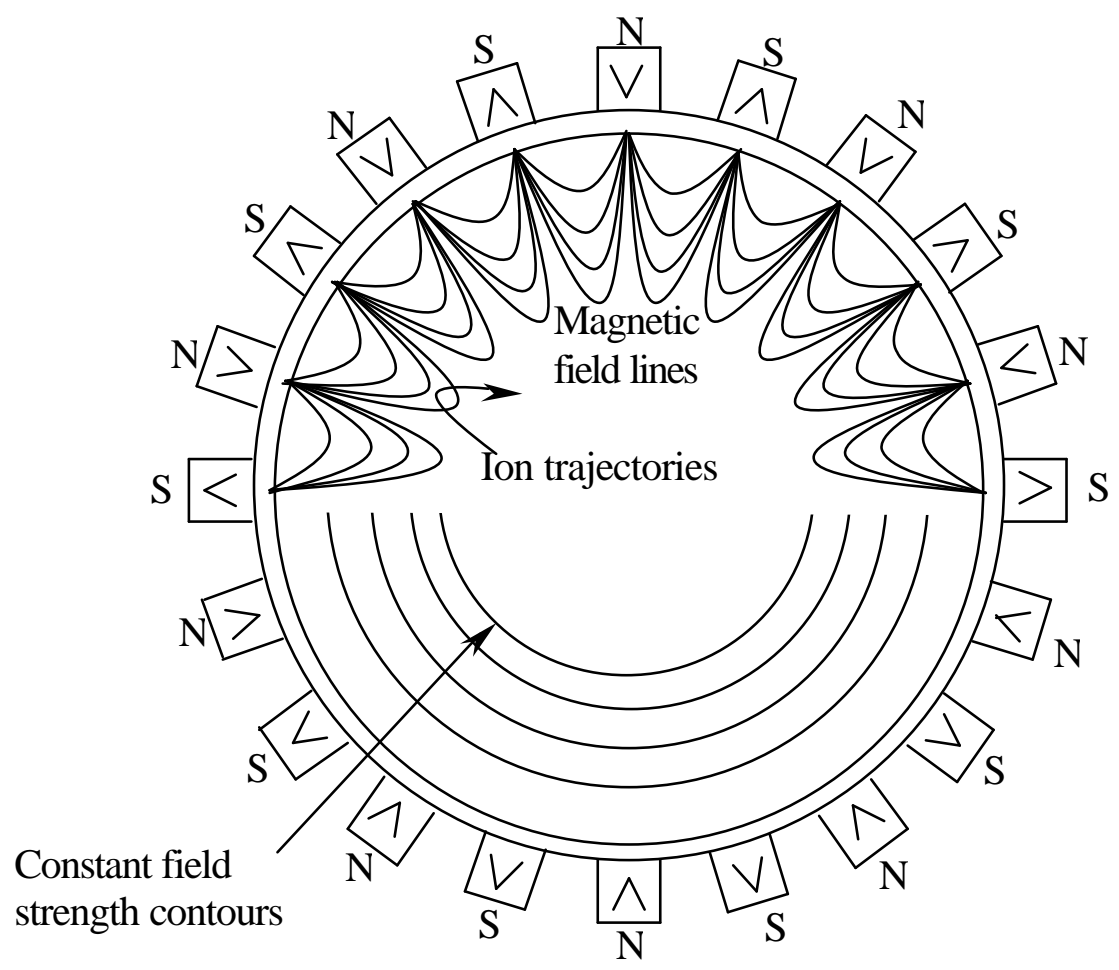

Figure 2.4 Magnetic multicusp confinements in cylindrical geometry, illustrating the magnetic field lines and the constant B surfaces near the circumferential walls.

Figure 2.4 shows a cross-sectional view of the source chamber and the magnetic field distribution. A uniform plasma is achieved with the multicusp ion source by arranging the magnets around the source chamber in such a way as to generate line-cusp magnetic fields, as shown in Fig. 2.4. The magnetic field strength $\mathrm{B}$ is a maximum near the magnets and decays with distance into the chamber. Most of the plasma volume can be virtually magnetic field free, while a strong field can exist near the discharge chamber wall, inhibiting plasma loss and leading to an increase in plasma density and uniformity. Fig. 2.5 shows the radial density profile of a multicusp source. The capability of the 
multicusp source to produce large-area of uniform plasma enables one to generate multiple ion beams from a single source. The advantage of using one source over multiple sources is less probability of failure, which is proportional to $\mathrm{N}^{2}$ (N is the number of sources involved).

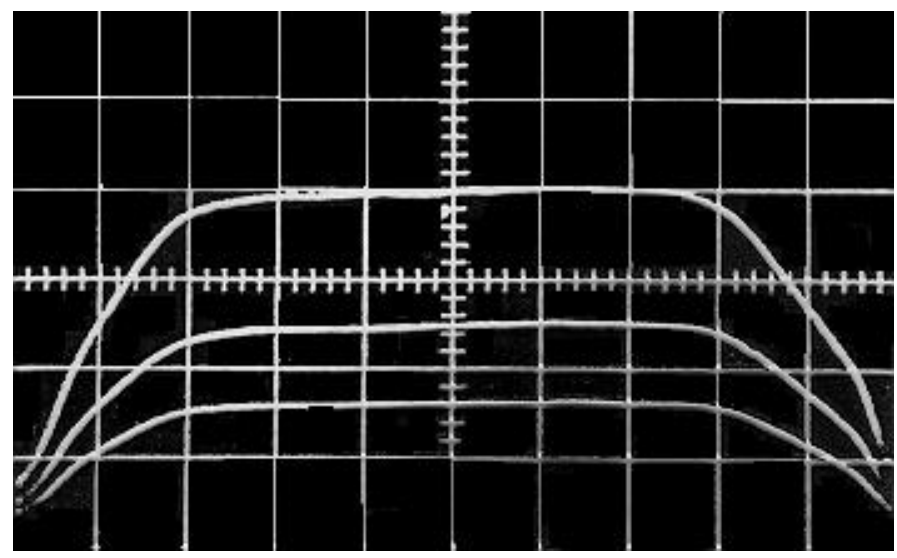

Figure 2.5 Radial density profile of a multcusp source.

\subsubsection{RF discharge plasma}

Multicusp ion source plasmas are generated by either filament dc discharge or RF induction discharge. RF discharge is chosen over filament discharge because it can generate a higher density and cleaner plasma with a longer lifetime. Tungsten filament cannot survive in oxygen plasma. Only RF discharge can be used to generate oxygen ions. Higher ion current density can be generated by RF induction discharge than filament discharge because higher power density can be applied. Filaments are limited to less than $250 \mathrm{~W}$ of power, while RF can go as high as 5 to $10 \mathrm{~kW}$. In a filament discharge plasma, filament material will be either sputtered or evaporated into the interior of the chamber and cause contamination. The emitted material could plug up the small 
extraction aperture of the FIB system as well. Quartz antenna is used in RF discharge plasma generation. As a result, much cleaner plasma is produced. Finally, the lifetime of an antenna is longer than a filament. Antenna lifetime greater than 500 hours continuous wave operation has been demonstrated. ${ }^{14}$

The RF antennas can be made of different materials. For example, metals such as copper, aluminum, and titanium have been used and can operate when left bare or coated with porcelain or quartz. The porcelain or quartz coated antenna can withstand the ion bombardment from the plasma and are protected against electrical as well as thermal damage. In addition, the coated antenna is energy efficient in plasma production since it eliminates the direct contact between the plasma and the metallic coil. Porcelain-coated antennas have been tested in hydrogen plasmas under cw operation for up to $20 \mathrm{~h}$ at an $\mathrm{rf}$ input power of $10 \mathrm{~kW}$ and tens of minutes at $20 \mathrm{~kW}$, without any sign of degradation of the antenna coating. At lower power levels $(\sim 5 \mathrm{~kW})$, antenna lifetime in excess of $260 \mathrm{~h}$ have been reported. ${ }^{15}$ However, if oxygen is used as the feeding gas, the porcelain antenna cannot survive more than $10 \mathrm{~h}$ of continuous operation. ${ }^{16,17}$ To address this issue, two kinds of quartz antennas had been tested: a quartz tube that had a silver wire inside and a quartz tube that had a titanium tube inside. The disadvantage of wire type antenna to tube type is that in case of antenna failure, the cooling water, which is flowing inside the quartz tube, leaks into the vacuum chamber. Titanium tube antenna is structurally more rigid. Thus antenna failures, which are caused by mechanical stresses of fragile quartz tubes, are greatly reduced. If for some reason antenna fails during the source operation, only air will be leaked into the source. From the operational point of view, this failure is much easier to handle compared to water leakage. ${ }^{14}$ 


\subsubsection{Axial energy spread}

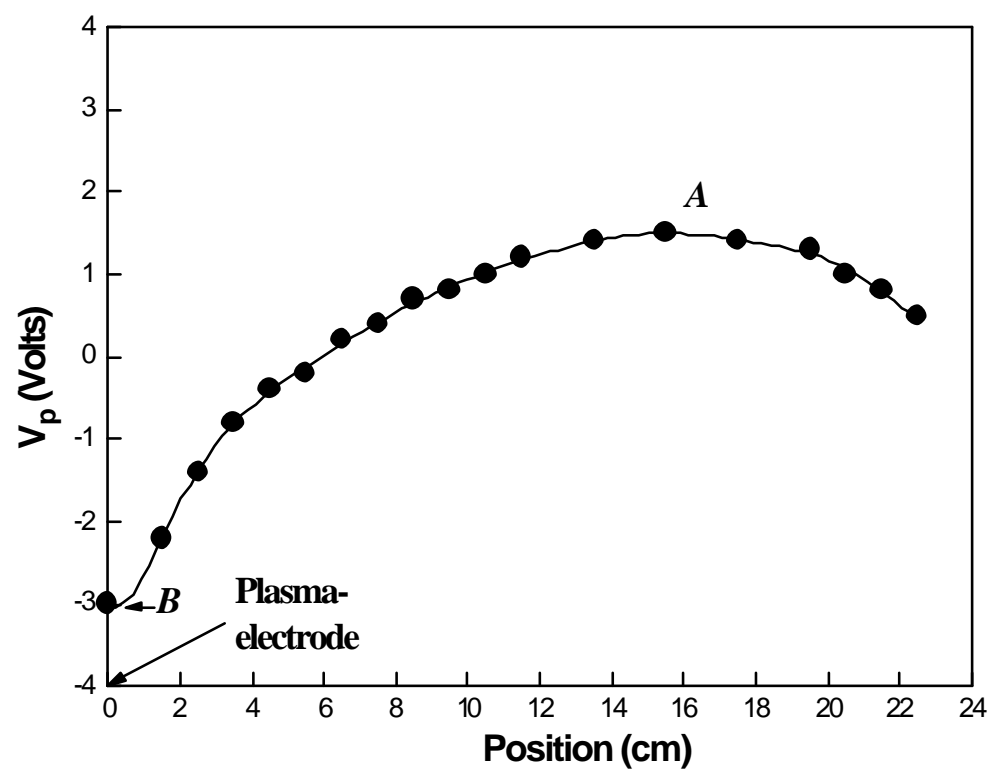

(a)

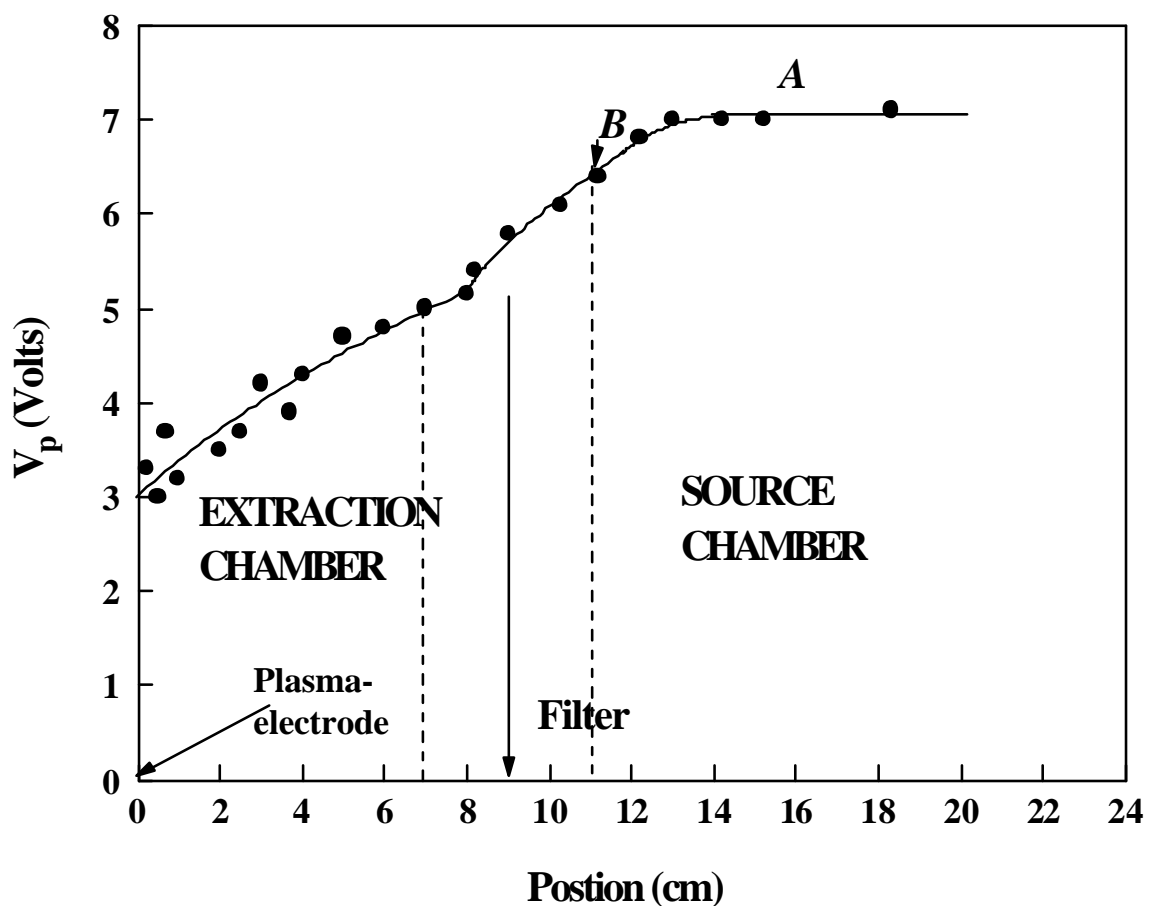

(b)

Figure 2.6 Axial plasma density (a) without and (b) with a magnetic filter. ${ }^{18}$ 
As a source for lithography application, one of the most important parameters is the energy spread. Low energy spread results in low chromatic aberration, which will ease the design of ion optics for low energy focus ion beam system.

Several factors can contribute to the energy spread. In the case without the magnetic filter, the axial plasma potential (Vp) profile in Fig. 2.6(a) shows that the plasma potential decreases monotonically towards the plasma electrode. $A$ and $B$ are the maximum and minimum plasma potential values where ions can be born. Ions born at position $A$ have more energy when they arrive at the exit aperture than ions born in positions $B$, given by the difference in potential between the two points. Positive ions generated at high $\mathrm{Vp}$ will reach the extractor as well as the ions created at lower potentials. Since the ions are generated at positions with different plasma potential, they will have a spread in axial energy when they arrived at the extraction aperture. In addition, ionization may take place in the plasma sheath of the extraction aperture, which may also lead to an increased energy spread. ${ }^{18}$

The magnetic filter placed in front of the extraction region as shown in Fig. 2.3 can reduce the axial energy spread of the ions. From the Vp distribution measurement in Fig. 2.6(b), the presence of filters created a region with a relatively uniform $\mathrm{Vp}$ profile in the discharge chamber region. Primary electrons are confined in the source chamber by the filter magnet fields as well as the multicusp field on the chamber walls. The axial potential gradient in the extraction region produces no effect on the energy spread. Since all the positive ions are produced within the source chamber region, they arrive at the plasma electrode with about the same energy due to the uniform $\mathrm{Vp}$ distribution. However, there is still a small potential gradient, given by the potential difference 
between point $A$ and $B$ between the center and the filter region that causes a small spread.

${ }^{18}$ The axial energy spread can be optimized to a value of $1 \mathrm{eV}$ or less ${ }^{19-21}$, which is much smaller than other type of plasma source and LMIS.

\subsection{Current density of Multicusp Plasma Ion source}

\subsubsection{Ion extraction from a plasma source}

The maximum ion current density that can possibly be extracted from the multicusp source follows the Child-Langmuir law,

$$
j_{i}=\frac{4}{9} \varepsilon_{0} \sqrt{\frac{2 e}{m}} \frac{U^{3 / 2}}{d}
$$

where $\mathrm{j}$ is the current density of ions in the plasma, $\mathrm{U}$ is the voltage between the first and the second electrode, $\mathrm{d}$ is the distance between the first two electrodes, $\mathrm{m}$ is mass of the ions, and $\varepsilon_{0}$ is the permissivity constant. As shown in Figure 2.7, there are three cases of ion extraction from plasma sources: (a) overdense plasma; (when the plasma density is relatively high and/or the extraction field is low, meniscus will be approximately spherical with its center of curvature inside the plasma) (b) intermediate plasma; (nearly planar under optimum conditions) and (c) underdense plasma (when the plasma density is low or, correspondingly higher extraction field, meniscus is spherical with the center of curvature outside the plasma). The miniscus shape of the plasma can be changed by adjusting the voltage and distance of the extraction lens. 


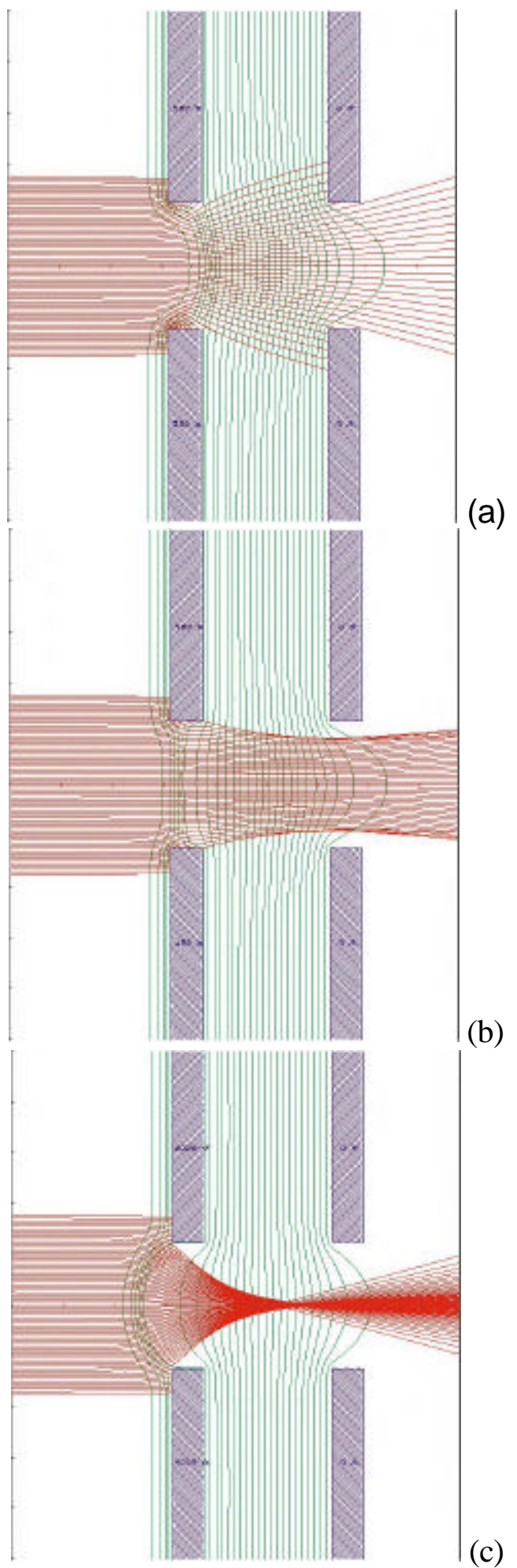

Figure 2.7 Plasma extraction: (a) overdense plasma, (b) intermediate density plasma, (c) underdense plasma. 
The current density of multicsup plasma ion source has been measured as a function of extraction energy, input RF power, and the source size. Figure 2.9 shows the set up of measuring the current density of the source. The beam is extracted from the source through a $1-\mathrm{mm}$-diameter aperture and the current is detected by a Faraday cup, on which a pair of permanent magnet is mounted to prevent secondary electron from coming out.

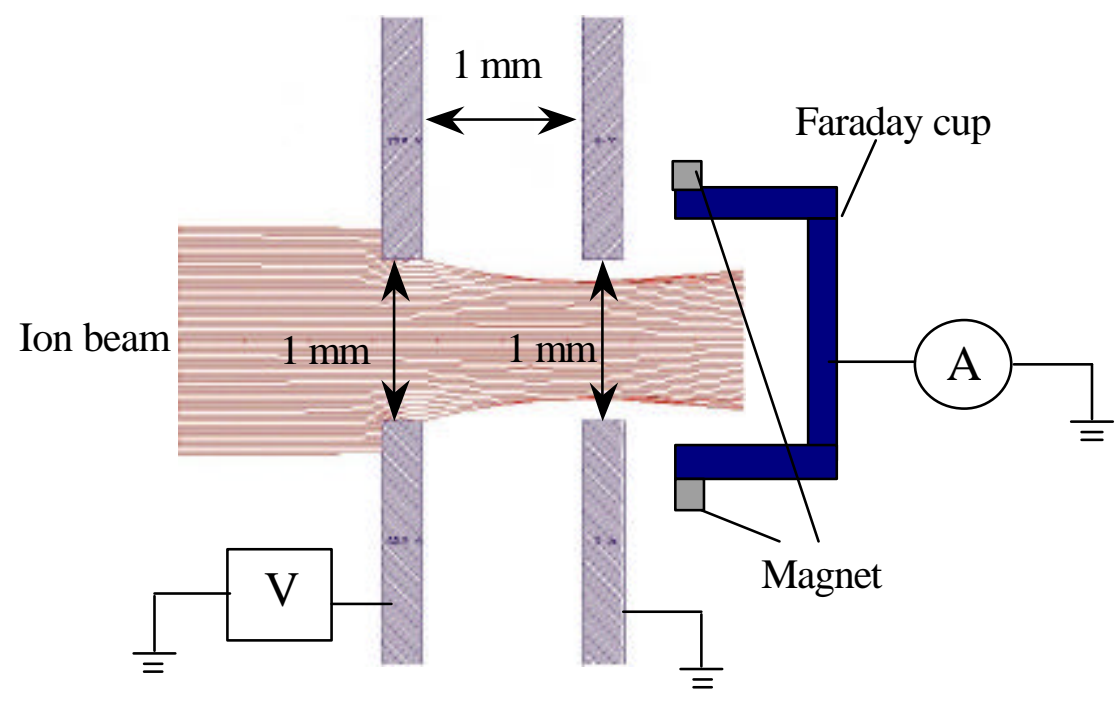

Figure 2.8 Current density measurement setup.

\subsubsection{Current density as a function of extraction voltage}

For a fixed extraction gap, the current density increases with the extraction energy. When the applied extraction voltage is low, the plasma is overdense. Ion beams are extracted divergently and most of the current is blocked by the second electrode (Fig 2.7a). As the extraction voltage increases, the divergent angle becomes smaller and smaller, leading to increasing current through the second aperture. The current density as a function of extraction voltage is shown in Figure 2.9. 


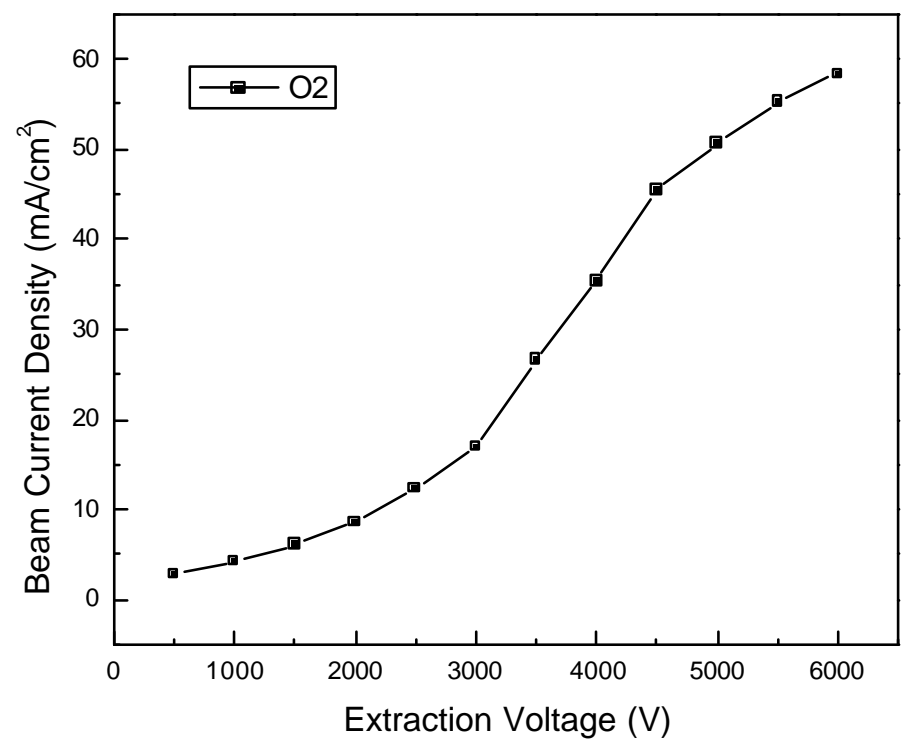

Figure 2.9 Oxygen ion current density as a function of the extraction voltage.

\subsubsection{Current density as a function of RF power}

The extracted oxygen ion current was measured as a function of input RF power at a gas pressure of $20 \mathrm{mTorr}$ in the source chamber and extraction voltage of $5 \mathrm{kV}$. The results are shown in Fig. 2.10. The higher the RF input power, the more plasma the source will produce. Therefore, the more ion current can be extracted. When the RF power is above $1500 \mathrm{~W}$, deviation from the linear trend is observed. Increases of RF power result in higher plasma density. Therefore, the plasma is changing from balanced condition to over dense condition as shown in Fig. 2.7. Beams become divergent and loss of current may occur. Higher extraction voltage is needed to balance the increased plasma density inside the source. At an RF input power of $2500 \mathrm{~W}$, the oxygen ion current density can be as high as $120 \mathrm{~mA} / \mathrm{cm}^{2}$. 


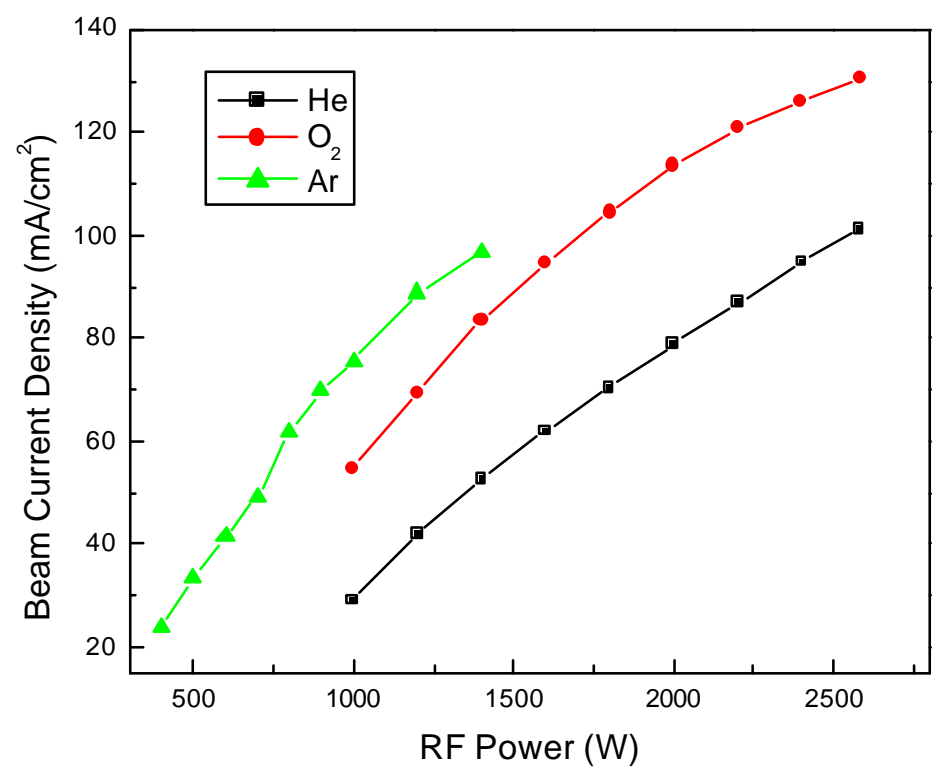

Figure 2.10 Oxygen ion current density as a function of input RF power.

\subsubsection{Current density as a function of source size}

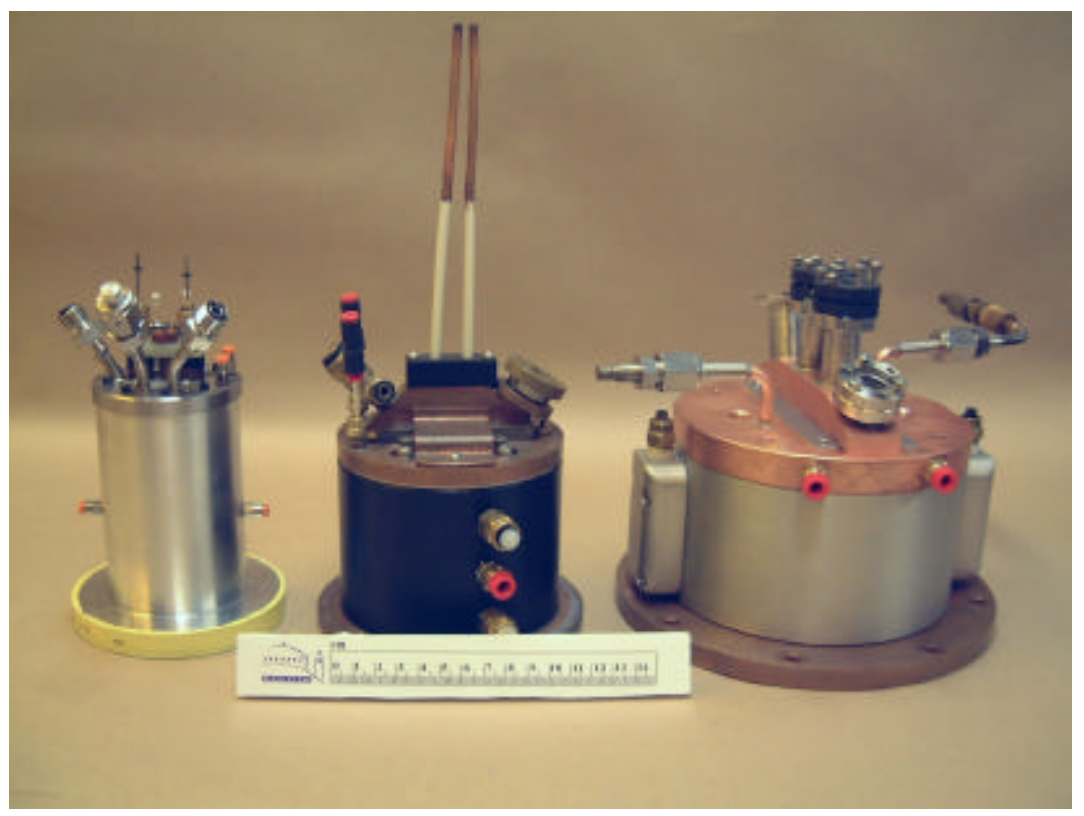

Figure 2.11 Picture of multicusp plasma source with chamber diameter of $5 \mathrm{~cm}, 7.5 \mathrm{~cm}$ and $10 \mathrm{~cm}$ (from the left to the right). 
Table 2.1 Antenna size for different multicusp plasma source.

\begin{tabular}{|c|c|c|c|}
\hline Source Diameter $(\mathrm{cm})$ & 5 & 7.5 & 10 \\
\hline Antenna Loop Diameter $(\mathrm{cm})$ & 3 & 4.5 & 6.8 \\
\hline
\end{tabular}

The size of the chamber of the multicusp plasma source also affects the extracted current denstity. Figure 2.11 shows a picture of three different size sources with their antenna dimension listed in Table 2.1: 5-cm-diameter source with a antenna loop diameter of $3 \mathrm{~cm}, 7.5-\mathrm{cm}$-diameter source with a antenna loop diameter of $4.5 \mathrm{~cm}$, and 10-cm-diameter source with a antenna loop diameter of about $7 \mathrm{~cm}$. As is know, an RF discharge is obtainable using an induction-coil antenna located inside the source. An azimuthal electric field is generated by the time-varying magnetic field inside the antenna loop. Electrons present in the gas volume are accelerated by the induced electric field, and quickly acquire enough kinetic energy to generate a high-density plasma by ionizing the background gas particles. This mode of operation is referred to as the inductively coupled mode, and it is desirable for achieving a high plasma density in the region from which the ion beams are extracted. If the antenna is too close to the wall, which happens to the 5-cm-diameter source, strong oscillating electric fields can easily be generated between the antenna and the source chamber, resulting in a capacitively coupled discharge in the source wall region. For this reason, it is difficult to operate a 5 -cm-diam source with a 3-cm-diam antenna in the inductively coupled mode, as compared to a 7.5$\mathrm{cm}$-diam source with a 4.5 -cm-diam antenna. For a big 10 -cm-diameter source, the power density in the source is lower than a $7.5-\mathrm{cm}$-diameter source at the same input RF power, which results in lower current density. Fig. 2.11 compares the extracted current density of 
these three different size sources. Apparently, the $7.5-\mathrm{cm}$-diameter source produces the highest current density at a given RF power.

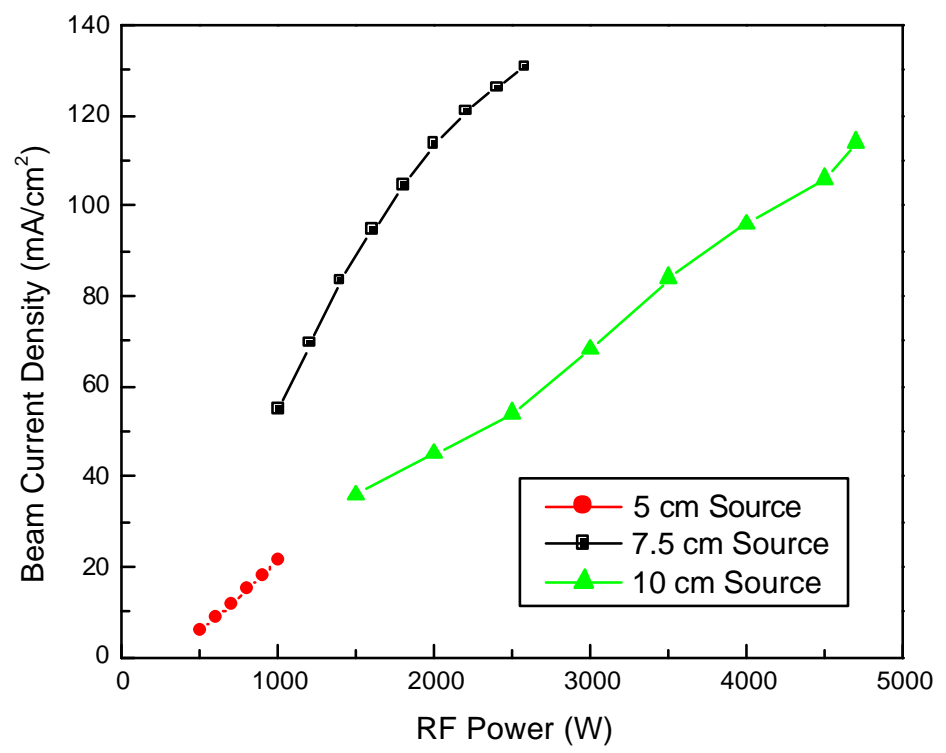

Figure 2.12 Comparison of oxygen ion current density for different size multicusp sources.

\subsection{Sources with external antenna}

For the RF source discussed above, plasma is generated using an internal antenna. Studies have shown that external antenna can also produce induction RF discharge. Figure 2.13 shows the schematic diagram of external antenna RF-driven source and a picture of the ignition of a hydrogen plasma using it. Instead of exposing to the plasma, the external antenna is wound around outside the quartz chamber. No ion bombardment occurs, which results in longer lifetime of the antenna. Even if the antenna fails, it won't affect the vacuum system. In addition, it is quite easy to exchange. External antenna source is easy to scale to size of as small as $1 \mathrm{~cm}$ diameter $^{22}$, and to size of as large as 
over $10 \mathrm{~cm}$ diameter. Therefore, it can be easily adopted as a source for either a single beam system or a multiple beam system.

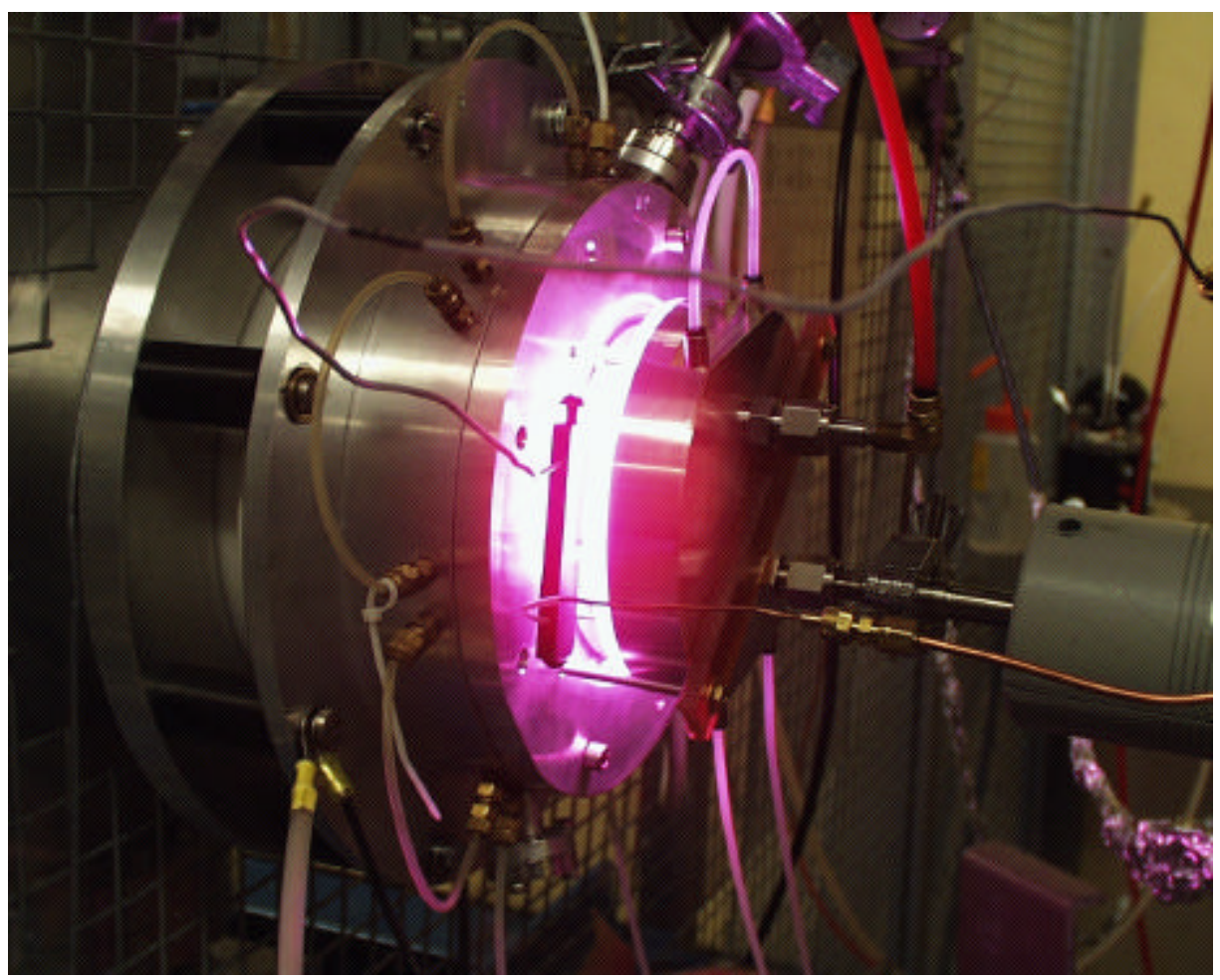

(a)

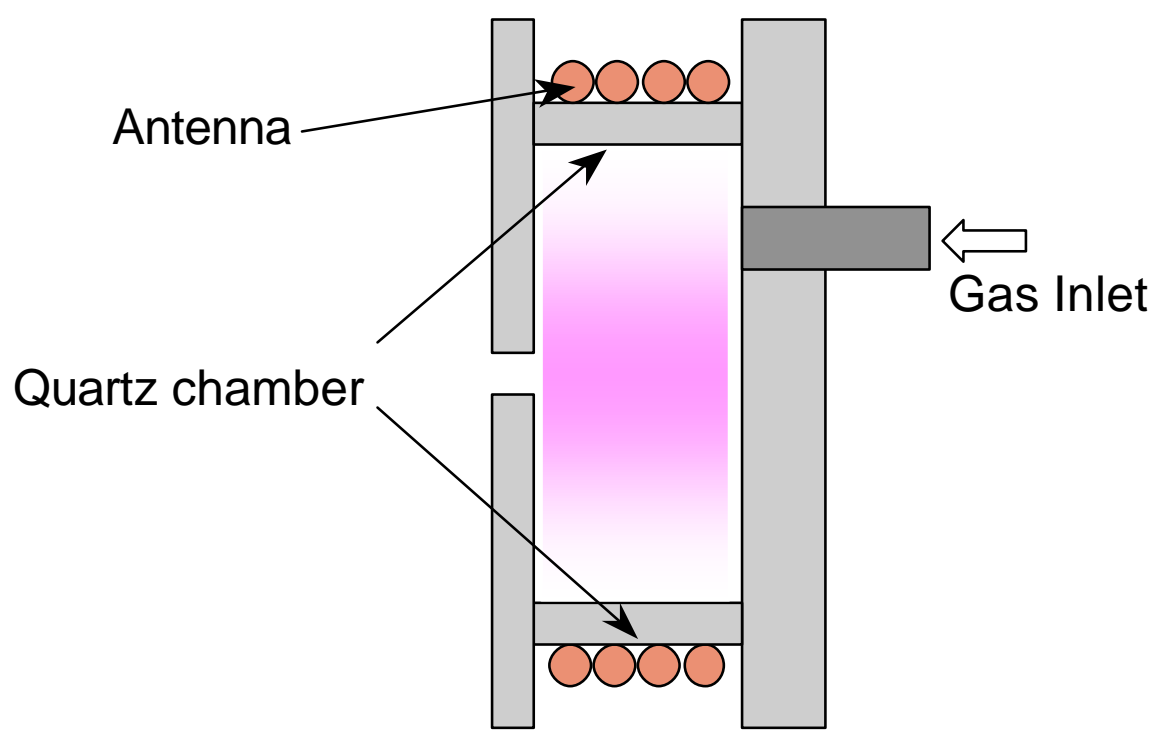

(b)

Figure 2.13 (a) The ignition of hydrogen plasma and (b) the schematic diagram of the external antenna inductive plasma source. 
Figure 2.14 shows the extracted beam current density from an external-antenna and internal-antenna generated hydrogen plasma, operating at the same extraction voltage. When operating at the same RF input power, the beam current density extracted from the external-antenna source is higher than that of the internal antenna source.

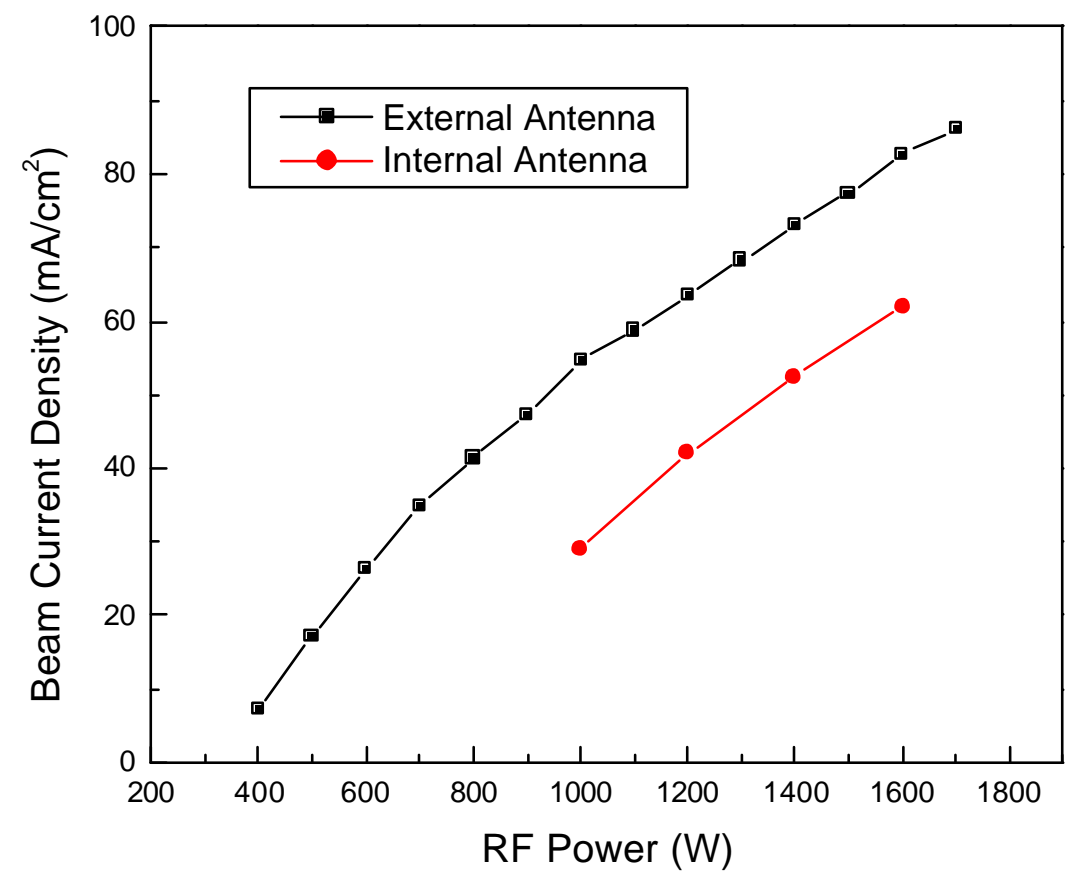

Figure 2.14 Comparison of hydrogen ion current density extracted from an external antenna and internal antenna source.

Simply by changing to negative extraction voltage, electrons can be extracted from the source using the same column. Figure 2.15 shows the electron current density produced by the external antenna source. At an input power of $2500 \mathrm{~W}$, electron current density of $2.5 \mathrm{~A} / \mathrm{cm}^{2}$ is achieved at $2500 \mathrm{~V}$, which is about 25 times larger than ion production. It indicates that such a source can also be used in electron projection lithography. 


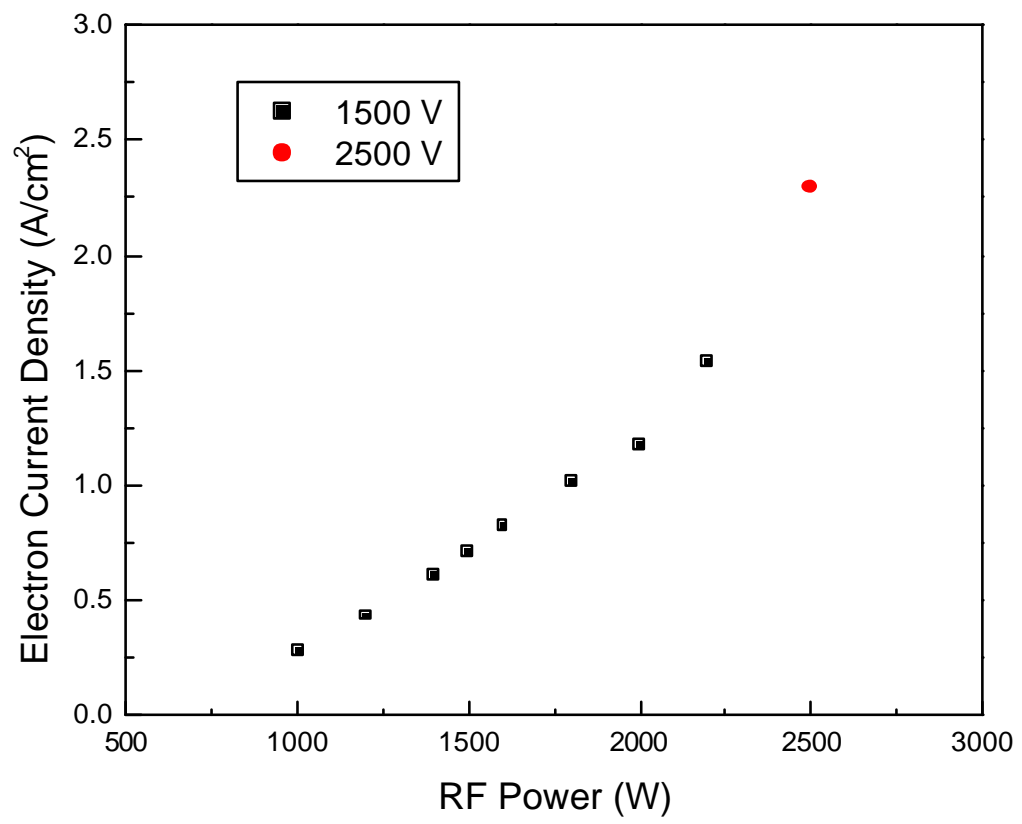

Figure 2.15 Electron current density extracted from an external antenna source.

\section{References:}

${ }^{1}$ J. Orloff, "High-resolution focused ion beams”, Rev. Sci. Instrum, 64(5), pp1105, 1993.

2 J. Melngailis, "Focused ion beam technology and applications", J. Vac. Sci. Technol. B5, pp469, 1987.

${ }^{3}$ E.Chason, S. T. Picraux, J. M. Poate, J. O. Borland, M. I. Current, T. Diaz de la Rubia, D. J. Eaglesham, O. W. Holland, M. E. Law, C. W. Magee, J. W. Mayer, J. Melngailis, and A. F. Tasch, "Ion beams in silicon processing and characterization", J. Appl. Phys, 81(10),pp6513, 1997.

${ }^{4}$ M. Kinokuni, H. Sawaragi, R. Mimura, and R. Aihara, "Development of wide range energy focused ion beam lithography system", J. Vac. Sci. Technol. B 16, 2484(1998).

${ }^{5}$ K. Edinger, J. Melngailis, and J. Orloff, "Study of precursor gases for focused ion beam insulator deposition", J. Vac. Sci. Technol. B 16, (1998) 3311. 
${ }^{6}$ L. E. Erickson, P. Schmuki, G. Champion, "Maskless deposition of gold patterns on silicon", J. Vac. Sci. Technol. B 16, (1998) 3198.

7 S. Nagamachi, Y. Yamakage, M. Ueda, and H. Maruna, J. Ishikawa, "Focused ionbeam direct deposition of metal thin film", Rev. Sci. Instrum. 67(1996)2351.

8 A. J. DeMarco and J. Melngailis, "Contact resistance of focused ion beam deposited platinum and tungsten films to silicon”, J. Vac. Sci. Technol. B 19, (2001) 2543.

${ }^{9}$ R. G. Woodham and H. Ahmed, "Combined focused ion beam deposition system and scanning probe microscope for metal nanostructure fabrication and characterization", $J$. Vac. Sci. Technol. B 17, (1999) 3075.

10 A. D. Della Ratta, J. Melngailis, and C. V. Thompson, "Focused-ion beam induced deposition of copper”, J. Vac. Sci. Technol. B 11, (1993) 2195.

11 J. Yanagisawa, H. Nakayama, K. Oka, M. Nakai, F. Wakaya, Y. Yuba, S. Takaoka, K. Murase, and K. Gamo, "Characterization of Si-doped layer in GaAs fabricated by a focused ion beam/molecular beam epitaxy combined system”, J. Vac. Sci. Technol. B 15, (1997) 2930.

12 K.N. Leung, "Plasma sources for electron and ion beams", J. Vac. Sci. Technol. B 17, 2776 (1999).

13 K.N. Leung, "Radio frequency driven multicusp plasma sources (invited)", Rev. Sci. Instrum. 69, 998(1998).

14 J. Reijonen, M. Eardley, R. Gough, R. Keller, K. Leung, R. Thomae, D. Pickard, and W. D. Williams, "Improvement of the lifetime of radio frequency antenna for plasma generation”, Rev. Sci. Instrum. 71, 1134 (2000). 
15 S. T. Melnychuk, T.W. Debiak, and J. J. Sredniawski, "Operation of a cw rf driven ion source with hydrogen and deuterium gas", Rev. Sci. Instrum. 67, 1662(1996).

${ }^{16}$ Y. Lee, R. A. Gough, K. N. Leung, L. T. Perkins, D. S. Pickard, J. Vujic, L. K. Wu, M. Olivo, and H. Einenkel, "Quartz antenna for radio frequency ion source operation", Rev. Sci. Instrum. 69, 1023(1998).

17 Y. Lee, R. A. Gough, W. B. Kunkel, K. N. Leung, J. Vujic, M. D. Williams, D. Wutte, and F. L. Yang, "Lifetime enhancement of a multicusp ion source for lithography", Microelectronic Engineering 41/42, 241(1998).

${ }^{18}$ Y. Lee, L. T. Perkins, R. A. Gough, M. Hoffmann, W. B. Kunkel, K. N. Leung, M. Sarstedt, J. Vujic, M. Weber, and M. D. Williams, "Production of low energy spread ion beams with multicusp sources", Nuclear Instruments and Methods in Physics Research A 374, 1 (1996).

${ }^{19}$ Y. Lee, R. A. Gough, W. B. Kunkel, K. N. Leung, L. T. Perkins, D. S. Pickard, L. Sun, J. Vujic, M. D. Williams, D. Wutte, Alfred A. Mondelli, and Gerhard Stengl, "Axial energy spread measurements of an accelerated positive ion beam”, Nuclear Instruments and Methods in Physics Research A 385, 204 (1996).

${ }^{20}$ Y. Lee, R. A. Gough, W. B. Kunkel, K. N. Leung, L. T. Perkins, D. S. Pickard, L. Sun, J. Vujic, M. D. Williams, and D. Wutte, "Ion energy spread and current measurements of the rf-driven multicusp ion source", Rev. Sci. Instrum. 68, 1398(1997).

21 Y. Lee, R. A. Gough, K. N. Leung, J. Vujic, M. D. Williams, and N. Zahir, "Co-axial multicusp source for low axial energy spread ion beam production", Nuclear Instruments and Methods in Physics Research A 433, 579 (1999).

${ }^{22}$ X. Jiang, Q. Ji, A. Chang, and K. N. Leung, "Mini RF-driven ion sources for focused 
ion beam systems", Rev. Sci. Instrum., to be published. 


\section{Chapter 3}

\section{Beam Brightness of Multicusp-Plasma Ion Source}

\subsection{Brightness}

The focal properties of a FIB system depend strongly on the source parameters. Usually two kind of brightness are used to characterize source or beam property.

\subsubsection{Source brightness}

The source brightness $\beta$, defined as angular current density divided by the effective or virtual source size $-\mathrm{d}_{\mathrm{v}}$, is taken as a measure of source quality. The virtual source size $d_{v}$, magnified or demagnified by the imaging system, limits the smallest focused spot size, while chromatic and geometric aberrations, caused by physical beam properties (e.g. energy spread and angular divergence), further broaden the beam. However, in order to take chromatic aberrations also into account, one compares the spectral brightness $\beta_{\mathrm{s}}$ which is defined as source brightness divided by source energy spread,

$$
\beta_{s}=\frac{I_{\Omega}}{d_{v} \Delta E}
$$


where $\boldsymbol{L}$ is the angular current density of the source, $\Delta \mathrm{E}$ is the axial energy spread of the ions ${ }^{1}$.

\subsubsection{Beam brightness}

Similar to source brightness, the beam brightness is defined as

$$
B=\frac{I_{\Omega}}{\pi r^{2}}=\frac{I}{\pi r^{2} \cdot \Omega}=\frac{J}{\Omega}
$$

where $h$ is the angular current density, $r$ is the radius of the beam spot at that plane, $I$ is the total beam current, $\Omega$ is the solid angle of the beam, and $\mathrm{J}$ is the current density at that plane. The solid angle into which a source emits ions is changed if a voltage Vs exists between the source and the point of measurement. This is almost always the case, and the specific brightness (normalized brightness $\beta=\mathrm{B} / \mathrm{Vs}$ is a more appropriate parameter. Nevertheless, the brightness B is most often quoted. ${ }^{2}$

\subsubsection{Comparison of brightness of different source}

Table 3.1 Characteristics of different source types, in terms of virtual source-size $D_{v}$, energy spread $\Delta \mathrm{E}$, angular current density $\mathrm{I}_{\Omega}$, brightness $\beta$, and spectral brightness $\beta_{\mathrm{s}}{ }^{1}$

\begin{tabular}{|c|c|c|c|c|c|}
\hline & $\mathrm{D}_{\mathrm{v}}(\mathrm{nm})$ & $\Delta \mathrm{E}(\mathrm{eV})$ & $\begin{array}{c}\mathrm{I}_{\Omega} \\
(\mathrm{mA} / \mathrm{Sr})\end{array}$ & $\begin{array}{c}\beta=\mathrm{I}_{\Omega} /\left(\pi \mathrm{d}_{\mathrm{v}}{ }^{2} / 4\right) \\
\left(\mathrm{A} / \mathrm{cm}^{2} \mathrm{Sr}\right)\end{array}$ & $\begin{array}{c}\beta_{\mathrm{s}}=\beta / \Delta \mathrm{E} \\
\left(\mathrm{A} / \mathrm{cm}^{2} \mathrm{SreV}\right)\end{array}$ \\
\hline $\begin{array}{c}\text { Conventional } \\
\text { Plasma Source }\end{array}$ & $5 \times 10^{4}$ & 5 & $3 \times 10^{3}$ & $10^{2}$ & 10 \\
\hline LMIS & 50 & 5 & 20 & $10^{6}$ & $10^{5}$ \\
\hline GFIS & $\sim 1$ & 1 & 0.1 & $10^{7}$ & $10^{7}$ \\
\hline Supertip GFIS & $\sim 1$ & 1 & 35 & $5 \times 10^{9}$ & $5 \times 10^{9}$ \\
\hline
\end{tabular}


Table 3.1 shows important parameters of conventional plasma source, LMIS, GFIS, and supertip GFIS. Conventional plasma source, although providing the highest current angular density $I_{\Omega}$, produce still the least brightness due to its largest virtual source size $(\sim 50 \mu \mathrm{m})$.

\subsubsection{Requirement of brightness}

The brightness of a multicusp-plasma ion source is a key issue for its application to maskless ion beam lithography. If an array of $1000 \times 1000$ independently controlled beamlets is used to expose a wafer, the brightness for each beamlet at the entrance of the objective lens needs to be $\sim 1000 \mathrm{~A} / \mathrm{cm}^{2} \mathrm{Sr}$, in order to achieve exposure throughput of sixty 300 -mm-diameter wafers per hour. ${ }^{3}$ The brightness of the source is proportional to the current density of the source. For a $80 \mathrm{~W}$ filament dc-discharge multicusp plasma source, one can achieve an output current density of $3 \mathrm{~mA} / \mathrm{cm}^{2}$ at a discharge power of 80 W. Scipioni et al. reported the image-side beam brightness (at $30 \mathrm{keV}$ ) of $1650 \mathrm{~A} / \mathrm{cm}^{2} \mathrm{Sr}$ for this kind of source. ${ }^{4}$ For a RF-driven source, Scott et al. recently reported the measured beam brightness of a 5-cm-diameter multicusp-plasma ion source to be less than $15 \mathrm{~A} / \mathrm{cm}^{2} \mathrm{Sr}$ at $3 \mathrm{keV}$ beam energy, using 5 - $\mu \mathrm{m}$-diameter extraction apertures, ${ }^{3}$ which falls far short of the brightness requirement. In this chapter, methods of improving source brightness and measurement of both spectral brightness and beam brightness will be presented. 


\subsection{Spectral brightness measurement}

\subsubsection{Experimental setup}

In order to measure the brightness of the source, ions are extracted from the source through a two-electrode column. The first electrode with an aperture of $1 \mathrm{~mm}$ in diameter is at the same potential as the wall of the ion source. The second electrode with an aperture of $0.1 \mathrm{~mm}$ is biased at $-600 \mathrm{~V}$ relative to the first electrode and serves as a collimator. Downstream, the beam can be further focused down.

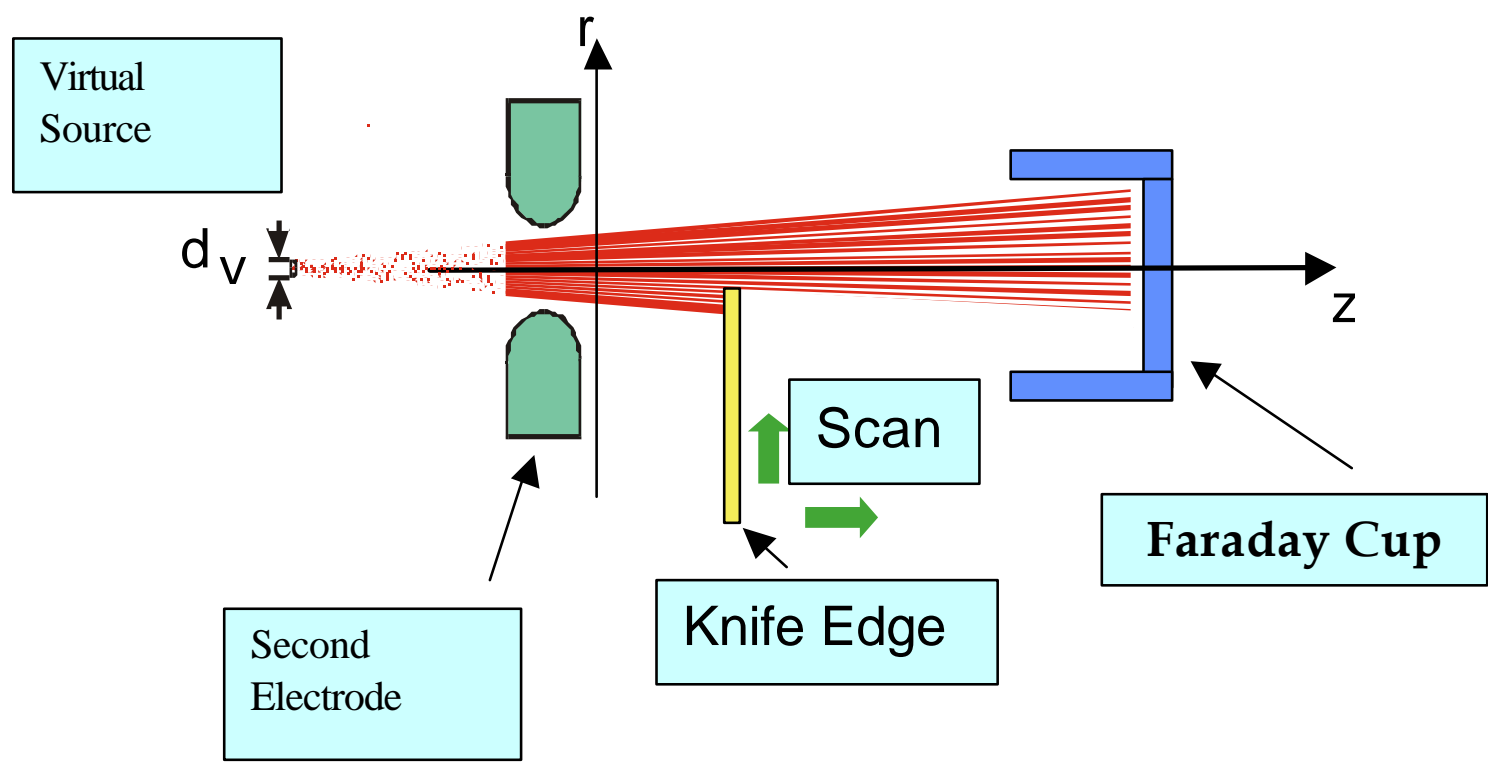

Figure 3.1 Schematic layout of the experiment for virtual source size measurement.

Argon gas was first used to test the source, because it has almost the same mass $\left(\mathrm{m}_{\mathrm{Ar}}{ }^{+}=40\right)$ as $\mathrm{O}_{2}{ }^{+}\left(\mathrm{m}_{\mathrm{O} 2}{ }^{+}=32\right)$. In this experiment, the filter was not mounted in the multicusp plasma source; therefore the energy spread was expected to be about $6 \mathrm{eV}$. In order to evaluate the $\mathrm{Ar}^{+}$beam brightness, a silicon knife edge controlled by a Burleigh inchworm X-Y-Z stage and a Faraday cup were inserted after the first two extraction 
electrodes for current distribution and virtual source size measurements. The experimental arrangement is shown schematically in Figure 3.1. The silicon knife edge can move in $\mathrm{x}, \mathrm{y}$, and $\mathrm{z}$ direction in steps of $50 \mathrm{~nm}$. The beam current distribution was measured by scanning the silicon knife edge across the beam and recording the beam current collected by the Faraday cup as a function of $r$.

\subsubsection{Results and discussion}

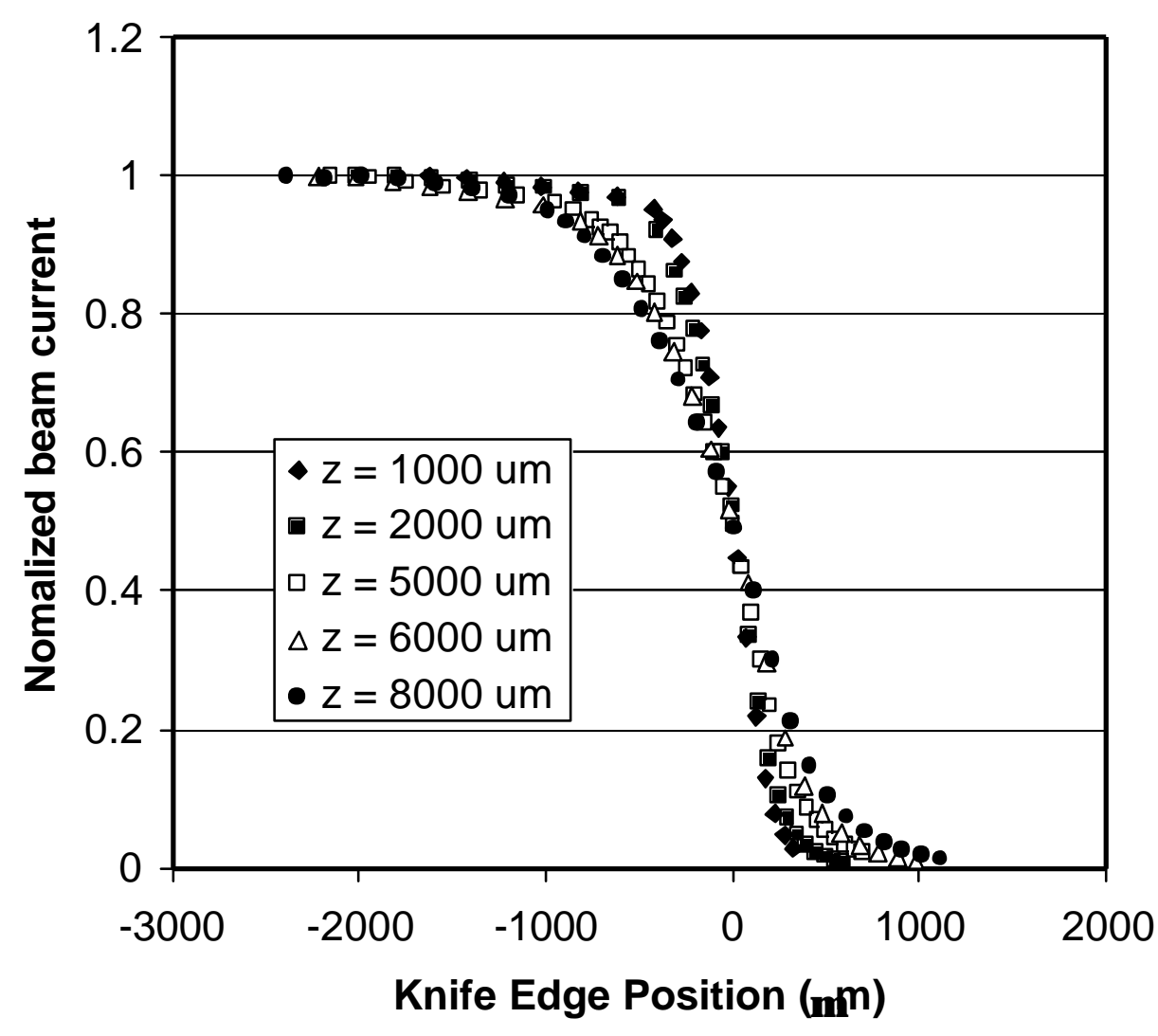

Figure 3.2 Collected beam current vs. knife edge position (r), for various $\mathrm{z}$ locations. Beam energy is $600 \mathrm{eV}$. 
Figure 3.2 is a plot of $\mathrm{Ar}^{+}$beam current distributions at different value of $\mathrm{z}$, for an extraction energy of $600 \mathrm{eV}$. The vertical axis is the normalized collected beam current. The horizontal axis is the radial position $\mathrm{r}$ of the knife edge relative to the second electrode. When the knife edge is not blocking the beam, the passing beam collected by the Faraday cup is $100 \%$ of the total extracted beam which is about $0.1 \mu \mathrm{A}$. When the knife edge totally blocks the beam, none of the $\mathrm{Ar}^{+}$ions can reach the Faraday cup and thus the collected beam current collected is zero. All the beam current distribution curves intersect at the same point where the passing beam current is $50 \%$. The rise of the beam distribution from $20 \%$ to $80 \%$ is used to define the beam size. From Fig. 3.2, the beam size increases as the position of the silicon knife edge moves away from extraction electrodes, indicating that the beam is divergent when extracted.

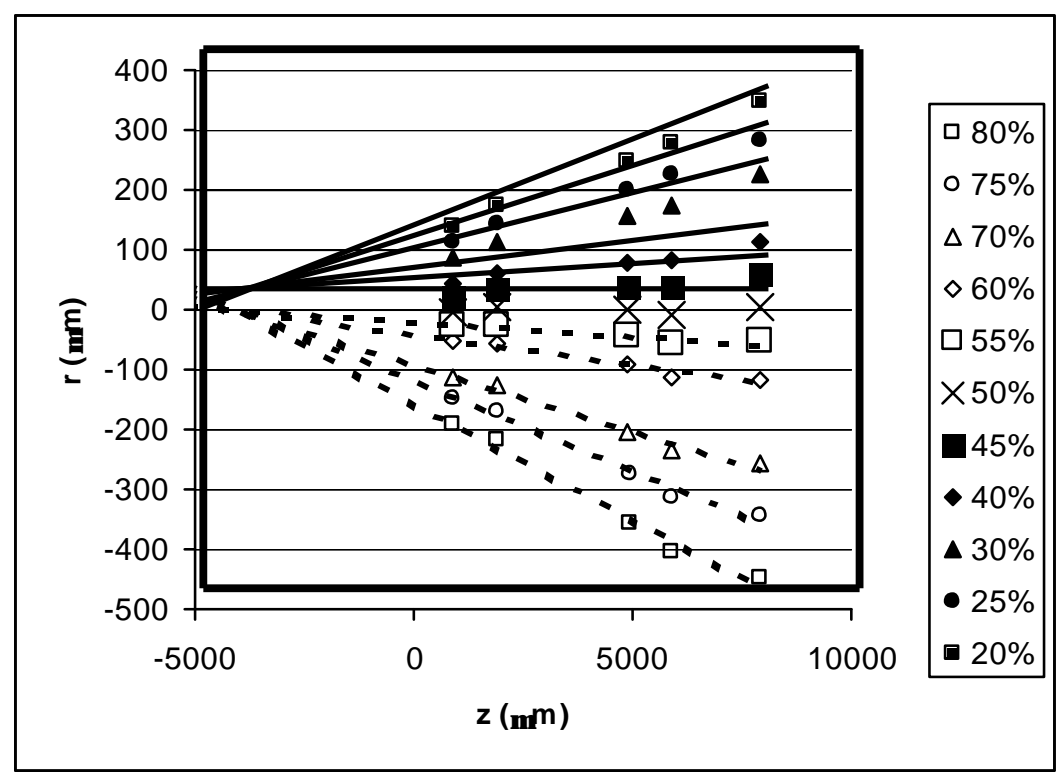




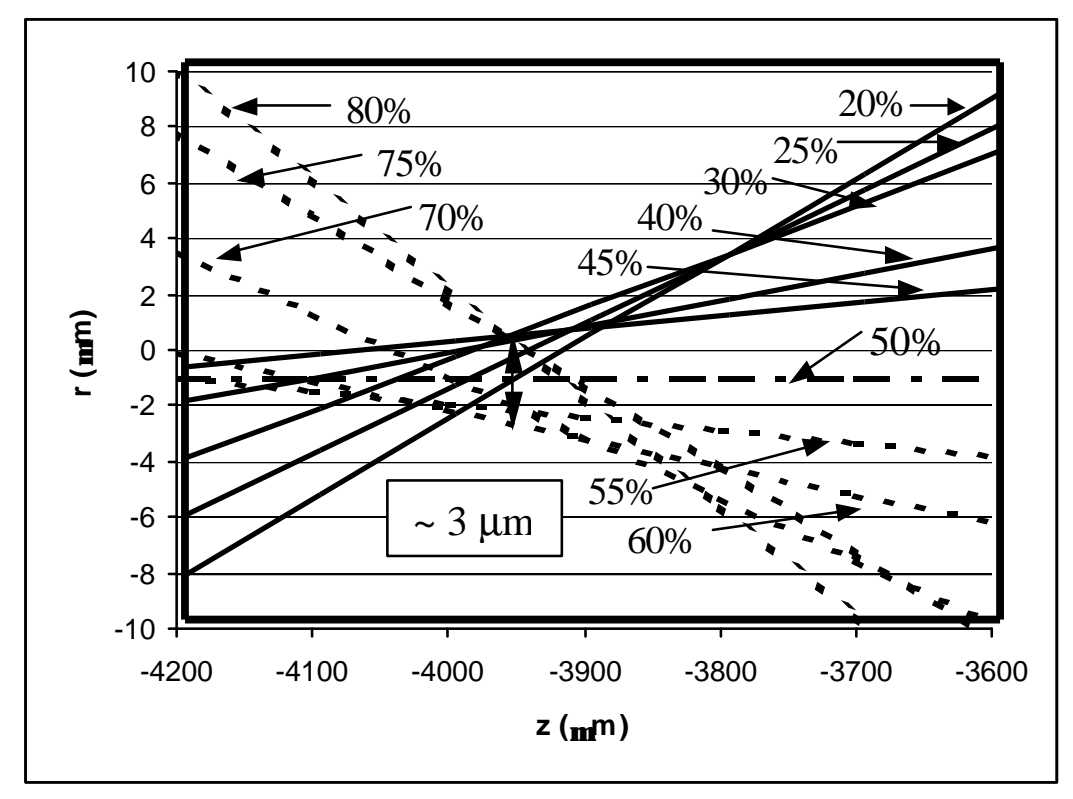

(b)

Figure 3.3 (a) Extracted $\mathrm{Ar}^{+}$beam envelope from $20 \%$ to $80 \%$ of the total beam. Straight lines were used to fit beam trajectories in the field-free region. (b) Zoom-in picture of the virtual source region. The virtual source size of the new FIB system is about $3 \mu \mathrm{m}$ in diameter.

When the beam enters a field-free region, the trajectory of the beam should be a straight line. The virtual source is the minimum width of the beam envelope when these beam trajectories are projected backwards. Fig. 3.3(a) shows the beam envelope of the extracted beam. The data points on the same beam trajectory in Fig. 3.3 were derived from Fig. 3.2 with the same normalized beam current at different knife edge positions. Straight lines (least mean square method) were used to fit the beam trajectories. By 
extrapolating these lines backwards to their intersection, the virtual source size was found from the smallest width to be about $3 \mu \mathrm{m}$. Fig.3.3(b) shows the zoom-in plot of the virtual source region.

Based on the virtual source size measurement, the angular current density of the extracted $\mathrm{Ar}^{+}$beam is $84.6 \mu \mathrm{A} / \mathrm{Sr}$ and the virtual source is about $3 \mu \mathrm{m}$ in diameter. Considering that the extractor also has a lens effect, the measured virtual source size using the method described above can be expressed as the following if only the chromatic aberration is considered:

$$
d_{v_{-} \text {measured }}^{2}=d_{v}^{2}+d_{c h r}^{2}
$$

where

$$
d_{c h r}=C_{c} \frac{\Delta E}{E} \alpha
$$

$\mathrm{C}_{\mathrm{c}}$ is the chromatic aberration coefficient, $\Delta \mathrm{E}$ is the energy spread and $\alpha$ is the beamlimiting aperture half angle ${ }^{5}$. Since the energy spread of the multicusp source including the magnetic filter can be reduced to about $1 \mathrm{eV},{ }^{6}$ the actual virtual source size is expected to be smaller than $1 \mu \mathrm{m}$. The spectral brightness defined in equation (1) that this new FIB system can achieve achieved at extraction energy of $600 \mathrm{eV}$ is better than $10^{4}$ $\mathrm{A} / \mathrm{cm}^{2} \cdot \mathrm{Sr} \cdot \mathrm{eV}$

\subsection{Beam brightness measurement}

As shown in Eqn. (2), for a fixed solid angle, higher current density at a given plane results in higher brightness. Two approaches have been taken to improve the brightness of a multicusp-plasma ion source: increasing the total beam current extracted from the 
source, and reducing the focused beam spot size. First, a larger source $(7.5 \mathrm{~cm}$ in diameter) is employed to achieve higher source current density. Second, a counter-bored extraction aperture is used to achieve smaller focused beam spot size with much reduced aberration. With increased RF power, the brightness of the multicusp-plasma ion source can be sufficiently high to make it suitable for application to high-throughput maskless lithography.

\subsubsection{Increasing total beam current}

Figure 3.4(a) shows the cross-sectional view of a RF-driven multicusp-plasma ion source. An RF discharge is generated using an induction-coil antenna located inside the source. As shown in Figure 3.4(b), an azimuthal electric field is generated by the timevarying magnetic field inside the antenna loop. Electrons present in the gas volume are accelerated by the induced azimuthal electric field, and quickly acquire enough kinetic energy to generate a high-density plasma by ionizing the background gas particles. This mode of operation is referred to as the inductively coupled mode, and it is desirable for achieving a high plasma density in the region from which the ion beams are extracted. If the antenna is too close to the wall, strong oscillating electric fields can easily be generated between the antenna and the source chamber, resulting in a capacitively coupled discharge in the source wall region. For this reason, it is difficult to operate a 5 cm-diam source with a $3-\mathrm{cm}$-diam antenna in the inductively coupled mode, as compared to a 7.5 -cm-diam source with a $4.5-\mathrm{cm}$-diam antenna.

Figure 3.5 compares the extracted $\mathrm{He}^{+}$beam current densities for the 7.5 -cm-diam and 5-cm-diam sources. The current density of the extracted beam increases with RF power 
for both sources. At $1 \mathrm{~kW}$ of $\mathrm{RF}$ input power, corresponding to the source operation condition in Ref. 3, the 7.5-cm-source provides $\sim 3$ times higher current density than the 5-cm-source. This difference may be attributed to the different modes of source operation as discussed in the last paragraph. The current density for the 7.5 -cm-diam source is over $100 \mathrm{~mA} / \mathrm{cm}^{2}$ at $2.5 \mathrm{~kW}$ of RF power, which is $\sim 10$ times larger than that of the 5-cm-diam source operating at $1 \mathrm{~kW}$ of $\mathrm{RF}$ power.

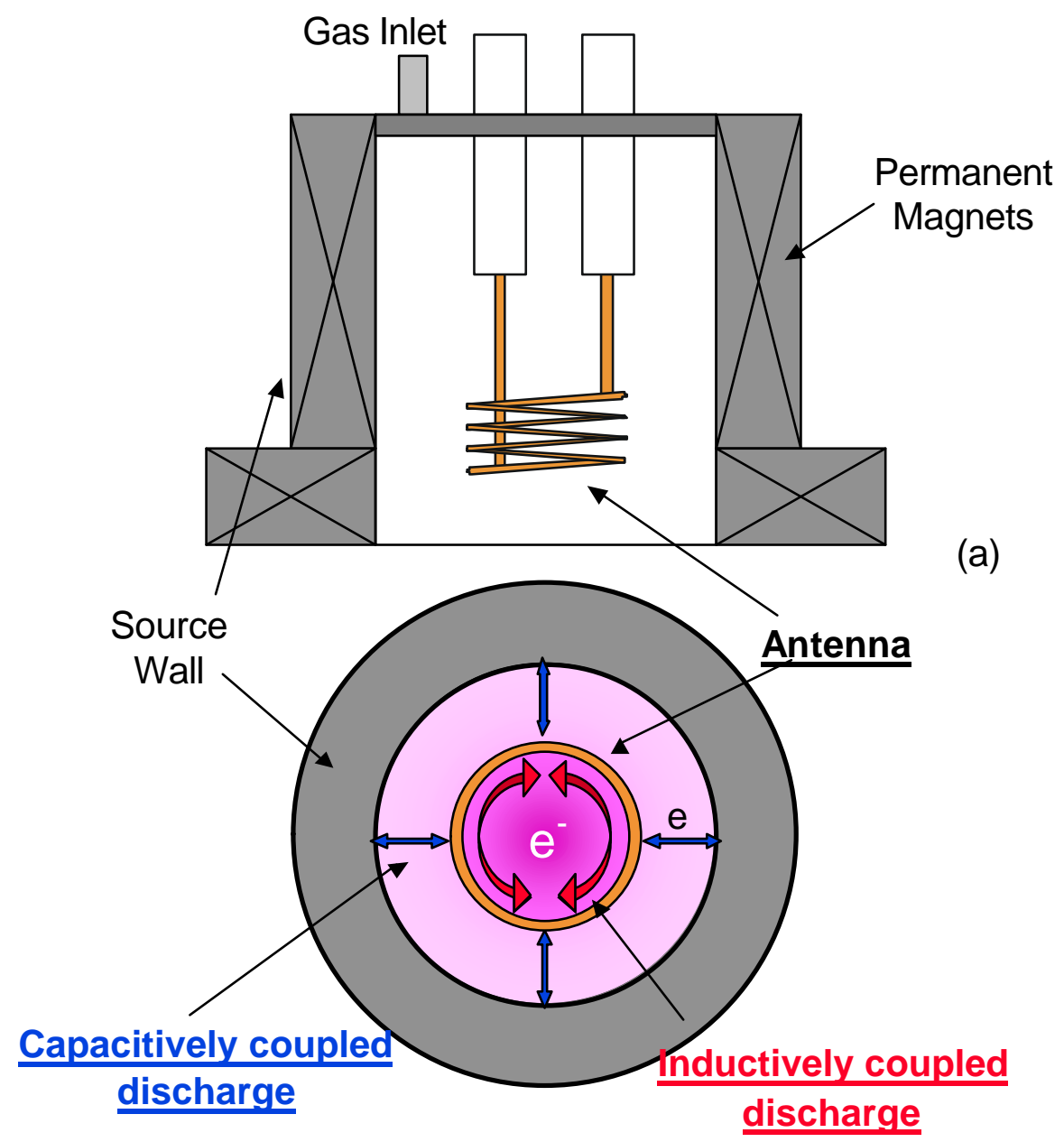

(b)

Figure 3.4 (a) Cross-sectional view of a RF-driven multicusp-plasma ion source. (b) Top view of the source. Two modes of plasma generation: capacitively coupled and inductively coupled discharge. 


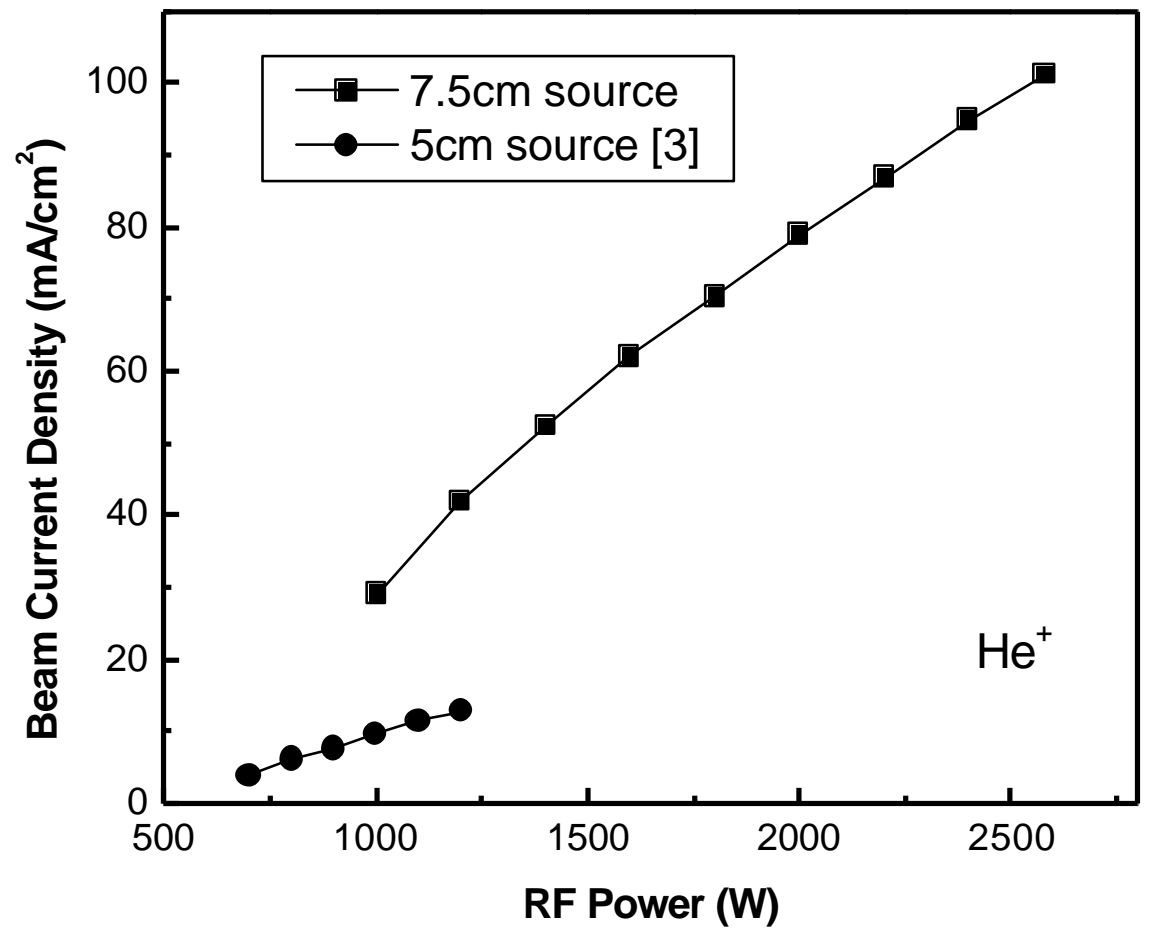

Figure 3.5 Comparison of extracted beam current densities for different size multicusp-plasma ion sources.

\subsubsection{Reducing focused beam spot size}

The effect of the two different extractor geometries on beam aberrations has been investigated: "through-hole" and counter-bored hole. As shown in Figure 3.7, the through-hole consists of a uniform-diameter $(50 \mu \mathrm{m})$ aperture, $250 \mu \mathrm{m}$ deep; the diameter of the counter-bored hole changes from $50 \mu \mathrm{m}$ (for the first $250 \mu \mathrm{m}$ of depth) to $300 \mu \mathrm{m}$ (for the second $250 \mu \mathrm{m}$ of depth) facing downstream. 
Simulations were performed using the Munro $\operatorname{code}^{7}$ and the results are summarized in Table 3.2. The aberration for the through-hole aperture is much larger than the focused Gaussian beam spot size. For an image $1 \mathrm{~mm}$ away from the extraction aperture, both geometries provide $\sim 4$ times reduction in image size. The through-hole generates almost one order of magnitude higher aberrations (spherical, coma, field curvature, astigmatism, and distortion) than the counter-bored hole. Thus, the counter-bored-hole geometry can substantially reduce the beam aberrations and hence the focused beam spot size. Ray tracing simulations were also performed using the SORAY program in Munro code ${ }^{7}$, and the results are shown in Fig. 3.6. For the through-hole geometry, the overall beam size at the image plane is around $36 \mu \mathrm{m}$, however, for the counter-bored-hole geometry, it is around $12 \mu \mathrm{m}$. They match with the results in Table 3.2 very well.

Table 3.2 Comparison of simulation results for a $50 \mu \mathrm{m}$ through-hole and counterbored extraction aperture. The image is $1 \mathrm{~mm}$ away from the extraction aperture.

\begin{tabular}{|c|c|c|c|c|c|c|c|c|}
\hline \multirow[b]{2}{*}{$\begin{array}{l}\text { Extraction } \\
\text { geometry }\end{array}$} & \multirow{2}{*}{$\begin{array}{c}\text { Overall } \\
\text { image } \\
\text { size } \\
(\mu \mathrm{m}) \\
\end{array}$} & \multirow{2}{*}{$\begin{array}{c}\text { Gaussian } \\
\text { image } \\
\text { size } \\
(\mu \mathrm{m}) \\
\end{array}$} & \multicolumn{6}{|c|}{ Aberration $(\mu \mathrm{m})$} \\
\hline & & & Spherical & Coma & $\begin{array}{c}\text { Field } \\
\text { curvature }\end{array}$ & Astigmatism & Distortion & Chromatic \\
\hline $\begin{array}{c}\text { Through } \\
\text { hole }\end{array}$ & 49 & 16.4 & 14.3 & 31.4 & 27.6 & 16.8 & 3.39 & 2.19 \\
\hline $\begin{array}{c}\text { Counter- } \\
\text { bored } \\
\text { hole }\end{array}$ & 13.9 & 13.1 & 1.58 & 2.99 & 2.31 & 1.13 & 0.24 & 1.49 \\
\hline
\end{tabular}

A simple exposure experiment was performed to verify the simulation results (Figure 3.7). A $2 \mathrm{keV} \mathrm{Ar}^{+}$beam generated by a multicusp ion source operating at $500 \mathrm{~W} \mathrm{RF}$ power was used to sputter a thin metal foil coated with dry erase ink for about 90 seconds. The target was located at $1 \mathrm{~mm}$ away from the extraction aperture. With a 
through-hole extraction aperture, a spot size of $56 \mu \mathrm{m}$ was obtained. In contrast, a spot size of $12.5 \mu \mathrm{m}$ was achieved with a counter-bored aperture. These results agree with the simulation results very well. With a counter-bored extractor, the focused beam spot size is about four times smaller, yielding another factor of ten increase in focused beam current density.

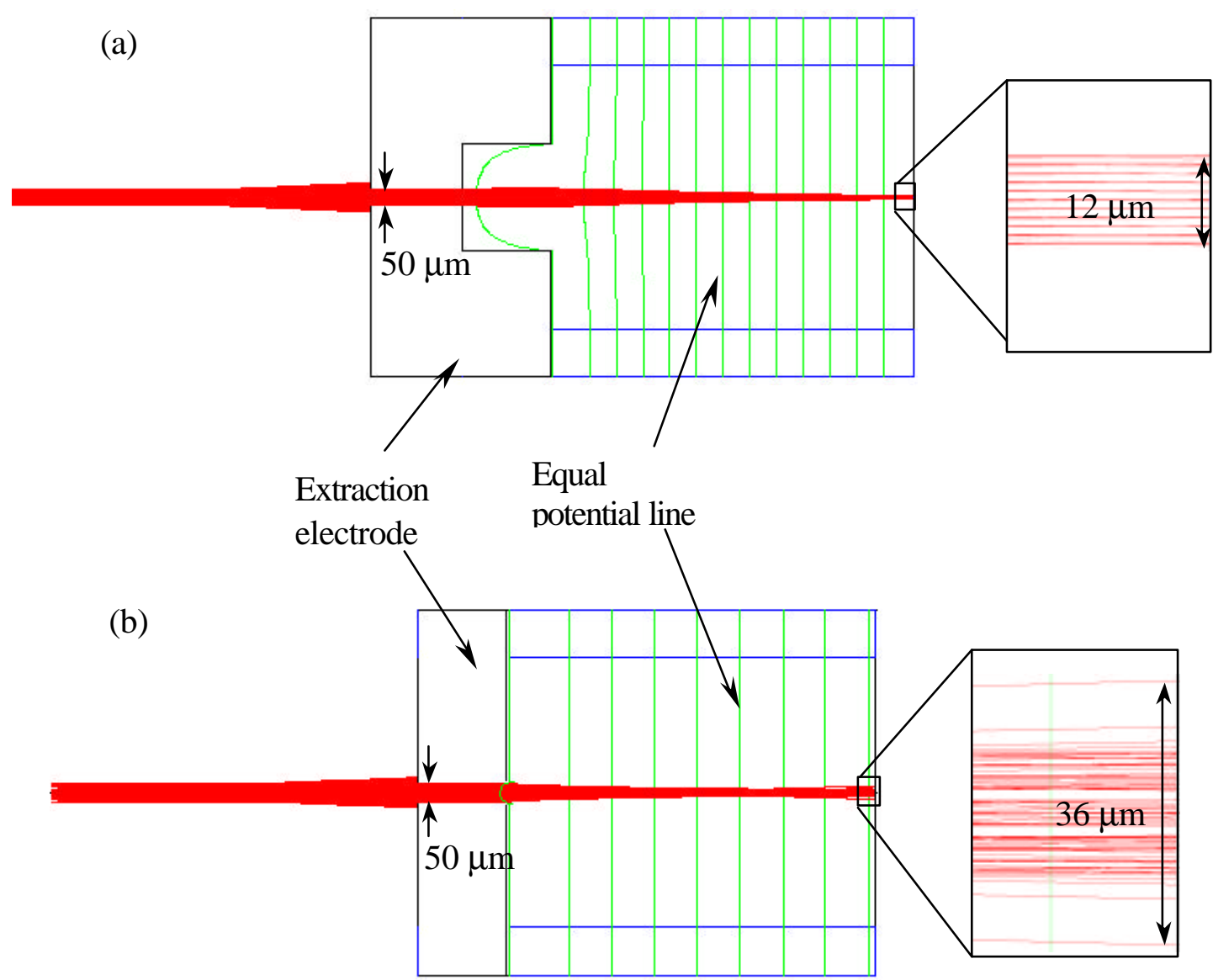

Figure 3.6 Ray tracing results using SORAY program in Munro code. (a) counterbored-hole geometry and (b) through-hole geometry. 


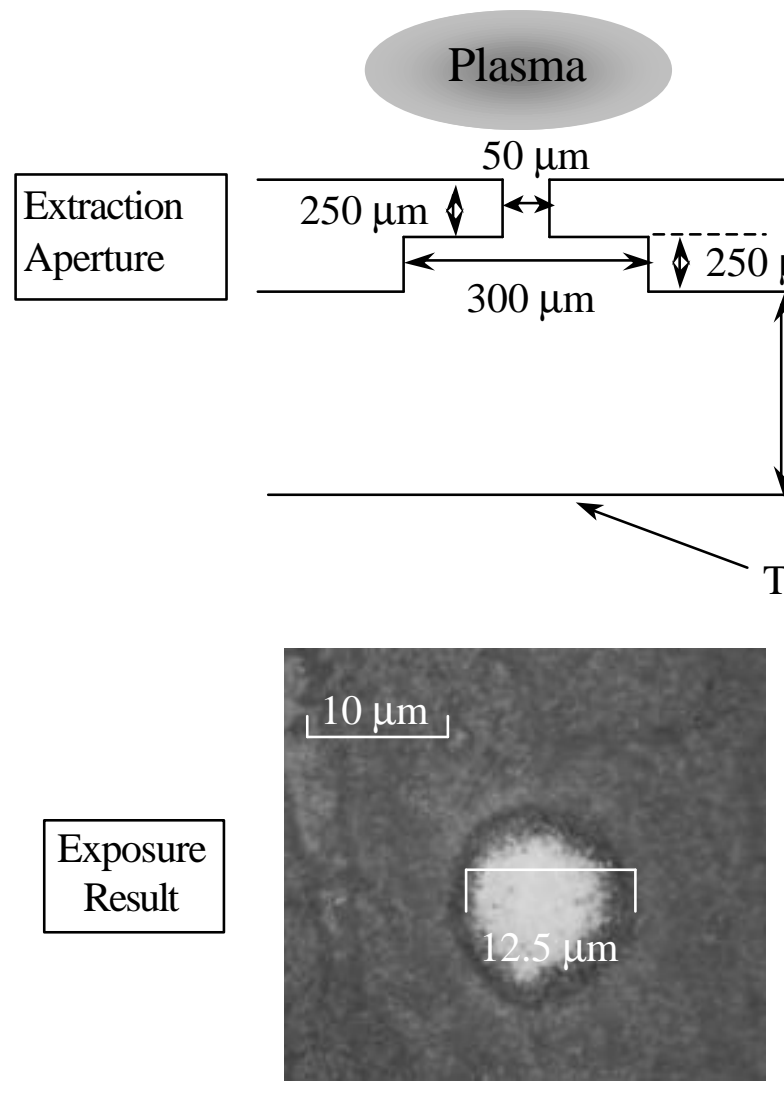

(a)

\section{Plasma} $50 \mu \mathrm{m}$
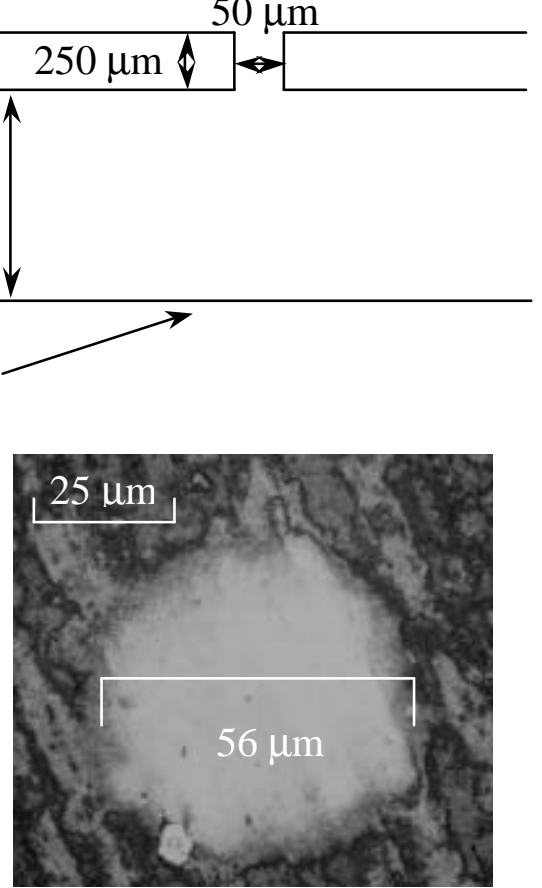

(b)

Figure 3.7 Comparison of exposure results using (a) counter-bored hole and (b) through hole as extraction aperture. $2 \mathrm{keV} \mathrm{Ar}^{+}$beams generated by a multicusp source at $500 \mathrm{~W}$ RF power were used to sputter a thin metal foil for about 90sec. The target was located $1 \mathrm{~mm}$ away from the extraction aperture.

\subsubsection{Experimental setup}

The setup for the brightness measurement is shown in Figure 3.8. A helium plasma, generated by a 7.5 -cm-diameter source, was used to illuminate a $50-\mu \mathrm{m}$-diameter counter-bored extraction aperture, which was at the same potential as the source chamber. The brightness analyzer was positioned $1 \mathrm{~mm}$ away from the extraction apertures to 
measure the beam profile. By scanning the voltages applied to deflector plates, the beam was swept over a $100 \mu \mathrm{m}$ slit located beyond the electrodes. Any current passing the slit was measured by a Faraday cup.

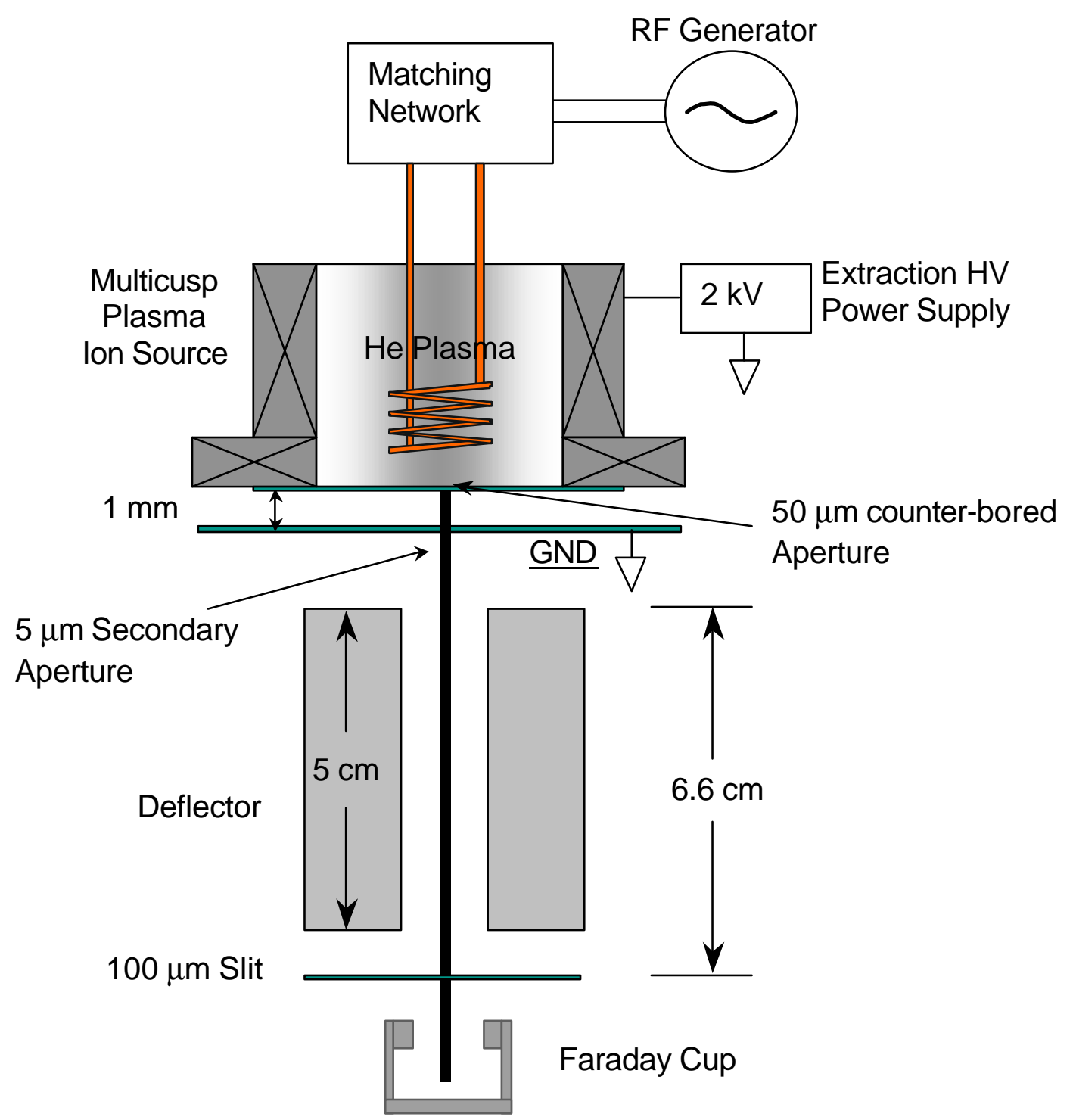

Figure 3.8 Experimental set-up for brightness measurement. 
The experimental setup was the same as for the prior work in Ref. 3, except for the following:

1. A 7.5-cm-diameter source was used instead of a 5-cm-diameter source. As mentioned above, the larger source is more easily operated in the inductively coupled mode, which provides a higher plasma density.

2. A $50 \mu \mathrm{m}$ counter-bored aperture was used to extract the ion beam instead of a 5 $\mu \mathrm{m}$ through-hole aperture. The focused beam spot size is smaller, due to reduced aberrations.

3. The beam energy was $2 \mathrm{keV}$ instead of $3 \mathrm{keV}$. No bias voltage was applied between the source and the extractor.

4. To prevent the secondary $5 \mu \mathrm{m}$ aperture from being damaged by the beam, the RF and extraction high-voltage power supplies were pulsed at a pulse width of 0.12 second and a duty factor of $2 \%$, except for the data measured at $2500 \mathrm{~W}$ RF power. At $2500 \mathrm{~W}$ RF power, the plasma is operated under $\mathrm{CW}$ mode, only the extraction high voltage power supply is pulsed. 


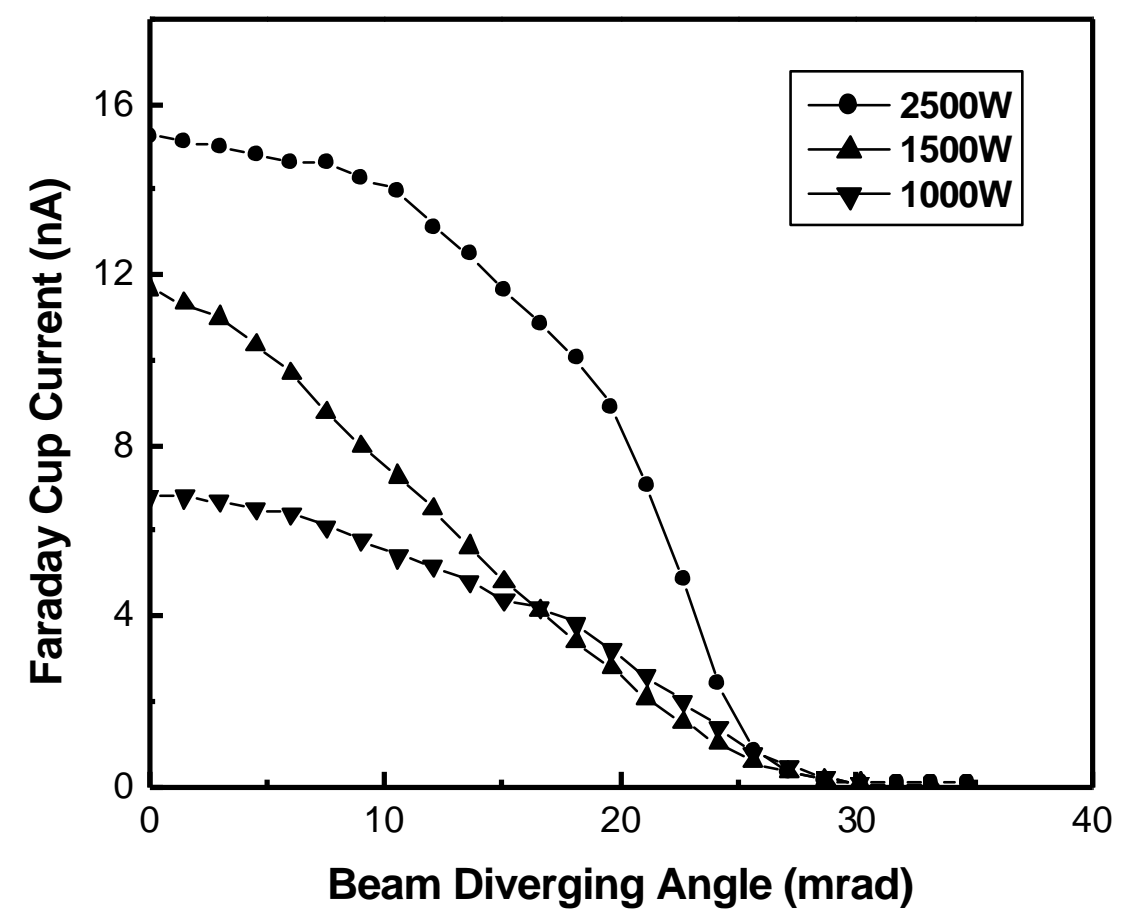

Figure 3.9 Beam profile at the $100 \mu \mathrm{m}$ slit, for 1.0, 1.5, and $2.5 \mathrm{~kW}$ RF power.

\subsubsection{Results and Discussion}

The beam profiles at the $100 \mu \mathrm{m}$ slit for RF power of 1.0, 1.5, and $2.5 \mathrm{~kW}$ are shown in Figure 3.9. The beam semi-angles are all $\sim 27 \mathrm{mrad}$ for the different power levels. The total beam current passing through the secondary $5 \mu \mathrm{m}$ aperture can be calculated by integrating the beam profile. Thus, the current density at the secondary aperture can be easily obtained. The beam brightness was determined using Eqn. (2), and the results are shown in Figure 3.10. It can be seen that the beam brightness increases with RF power, reaching $440 \mathrm{~A} / \mathrm{cm}^{2} \mathrm{Sr}$ at $2.5 \mathrm{~kW}$. Compared to the previous results ${ }^{3}$, a factor of 30 improvement in brightness is achieved. 


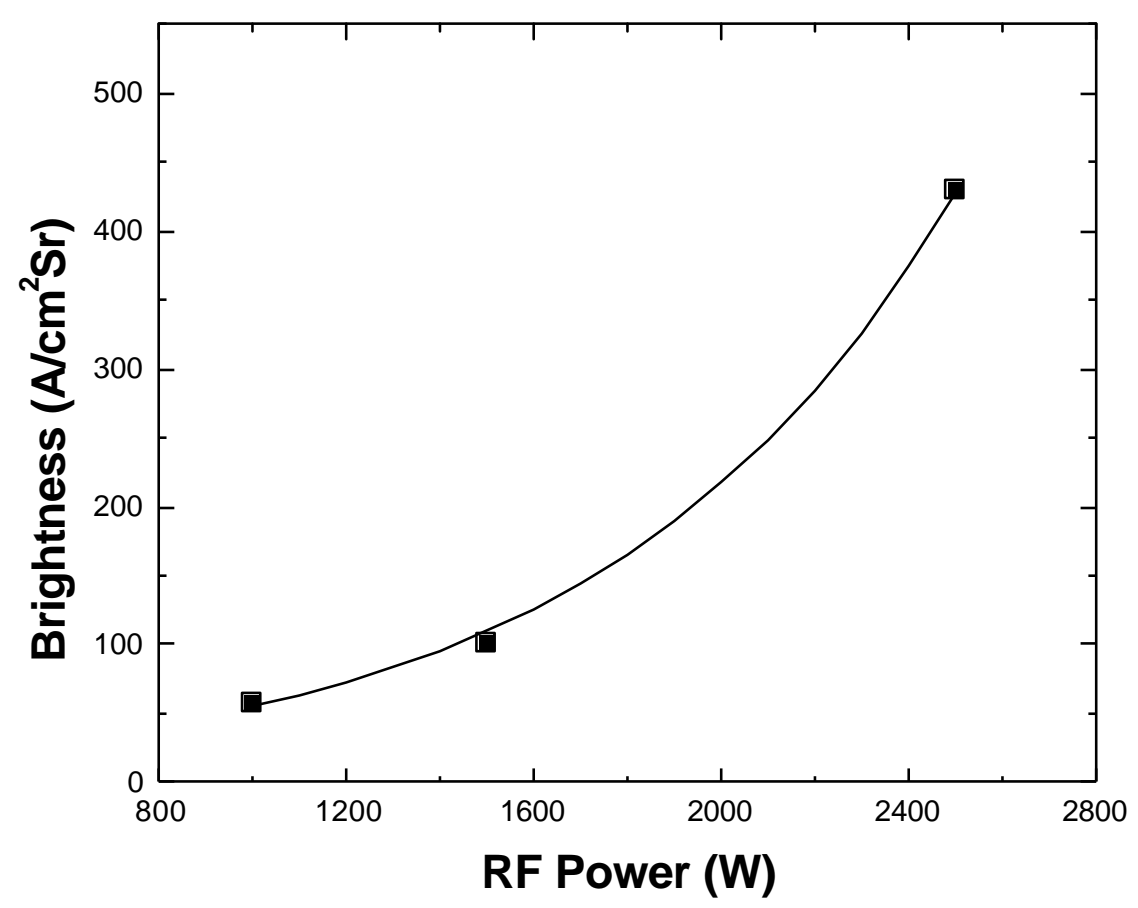

Figure 3.10 Brightness of $2 \mathrm{keV} \mathrm{He}^{+}$beams as a function of RF power.

\subsection{Summary}

In summary, it has been experimentally demonstrated that a beam of $\mathrm{Ar}^{+}$ions can be extracted from a multicusp plasma source using an all-electrostatic accelerator column. The extracted beam current after collimator is about $0.1 \mu \mathrm{A}$, and the spectral brightness of this ion source is better than $10^{4} \mathrm{~A} / \mathrm{cm}^{2} \cdot \mathrm{Sr} \cdot \mathrm{eV}$ at beam energy of $600 \mathrm{eV}$, which is about three orders of magnitude higher than that of the conventional plasma sources. The brightness of the beam extracted from a multicusp ion source can be high if it is operated in the inductively coupled mode and if the beam extraction system is optimized to reduce aberrations. For a 7.5-cm-diameter source with a 50- $\mu \mathrm{m}$ counter-bored extraction 
aperture, the measured brightness $\left(440 \mathrm{~A} / \mathrm{cm}^{2} \mathrm{Sr}\right)$ is significantly higher than in previously reported work. Additional increases in RF power as well as improved source design are being investigated to further improve the brightness in order to meet the requirement of $1000 \mathrm{~A} / \mathrm{cm}^{2} \mathrm{Sr}$ for high-throughput maskless lithography.

As shown in Section 3.3.1, the current density extracted from the source is as high as $100 \mathrm{~mA} / \mathrm{cm}^{2}$. If the demagnification factor of the downstream lens is around $10 \mathrm{X}$, the focused current density can reach $10 \mathrm{~A} / \mathrm{cm}^{2}$. A comparison of the spectral brightness for different types of sources is shown in Figure 3.11. ${ }^{1}$ The horizontal axis is the spectral brightness of the source, while the vertical axis is the focused current density at the target. The position of the FIB system described in this thesis work is marked by a bold circle on the map, with the spectral brightness determined by the virtual source size measurement and the possible focused current density which could be achieved at the target. The spectral brightness of the FIB system is about three orders of magnitude higher than that of conventional plasma source. The new FIB system with the multicusp plasma source can achieve almost the same spectral brightness as the LMIS can. By further optimizing the extractor, the extracted current angular density will increase, which results in a higher spectral brightness. By further optimizing the ion optics to get a larger demagnification factor, the focused spot size will decrease, which result in a larger focused current density. 


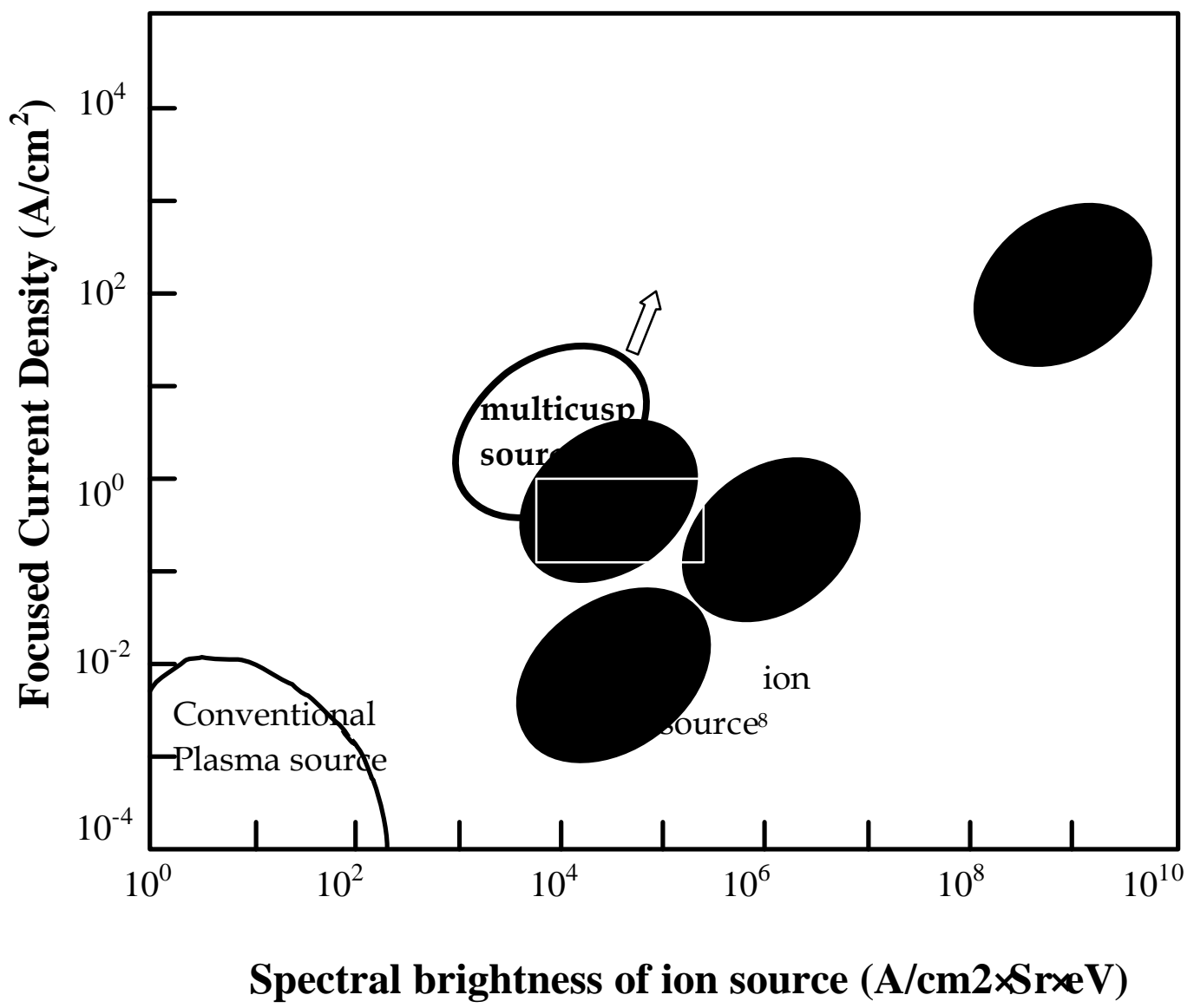

Figure 3.11 Comparison of spectral brightness with different ion sources.

\section{References:}

${ }^{1}$ Ch. Wilbertz, Th. Miller, and S. Kalbitzer, "Recent progress in Gas Field Ion Source technology", Procedding of SPIE, vol. 2194, 407(1994).

2 J. Melngallis, "Focused ion beam technology and applications", J. Vac. Sci. Technol. B 5, 469(1987). 
3 K.L.Scott, T.-J.King, K.-N.Leung, and R. F. Pease, "Characterization of multicuspplasma ion source brightness using micro-scale apertures", J. Vac. Sci. Technol. B 19, 2602 (2001).

${ }^{4}$ L. Scipioni, D. Stewart, D. Ferranti, and A. Saxonis, "Performance of multicusp plasma ion source for focused ion beam applications”, J. Vac. Sci. Technol. B 18, 3194 (2000).

5 J. Orloff, "High resolution focused ion beams", Rev. Sci. Instrum., 64, 1105 (1993).

6 Y. Lee, L. T. Perkins, R. A. Gough, M. Hoffmann, W. B. Kunkel, K. N. Leung, M. Sarstedt, J. Vujic, M. Weber, and M. D. Williams, "Production of low energy spread ion beams with multicusp sources", Nuclear Instruments and Methods in Physics Research A 374, 1 (1996).

${ }^{7}$ Provided by MEBS Ltd., 14 Cornwall Gardens, London SW7 4AN, England.

${ }^{8}$ S. K. Guharay, E. Sokolovsky, and J. Orloff, "Characteristics of focused beam spots using negative ion beams from a compact surface plasma source and merits for new applications", J. Vac. Sci. Technol. B 16, 3370(1998). 


\section{Chapter 4}

\section{Ion Optics for Maskless, Resistless Ion Beam Lithography System}

\subsection{Electrostatic lenses}

A conventional optical system consists of several rotationally symmetric (round) lenses which enable light to be focused and imaged. Ion optical imaging systems also consist of several rotationally symmetric ion lenses that enable the ions to be focused and imaged. Similar to a light optical imaging system, the action of the ion optical system is to transfer the ion image from the object plane to the image plane. In the paraxial approximation the ion optical system can form an ideal and clear image, but when aberration is present the image is distorted and unclear. ${ }^{1}$ 


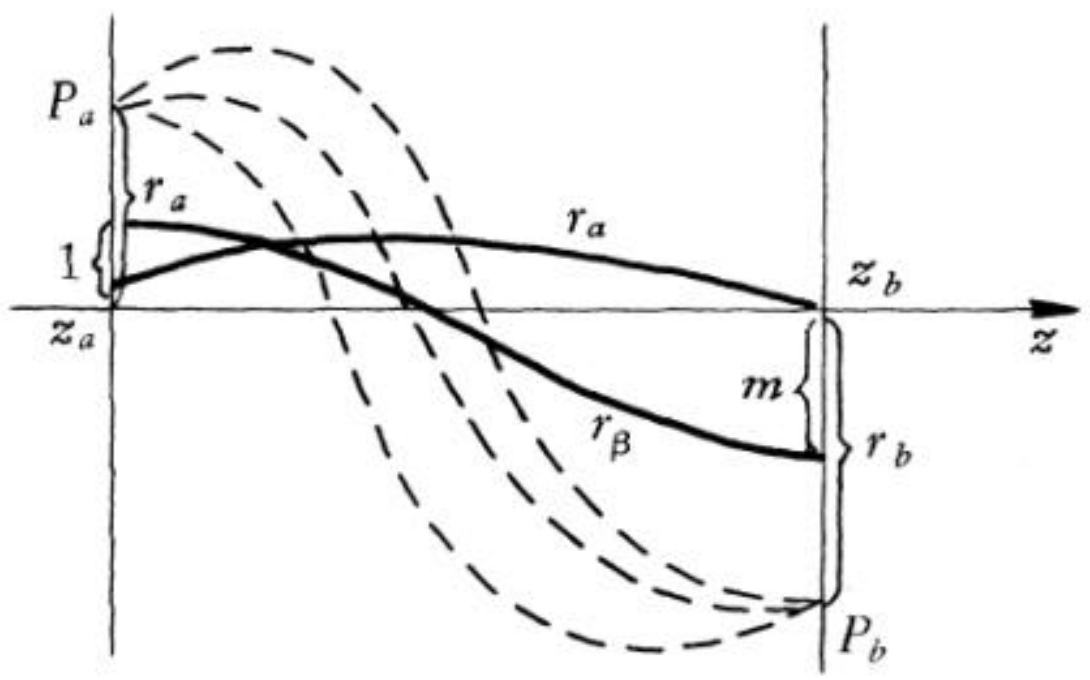

Figure 4.1 The ideal imaging Gaussian trajectory. ${ }^{2}$

As shown in Fig. 4.1, the object is at plane $\mathrm{z}=\mathrm{z}_{\mathrm{a}}$, and its Gaussian image plane is located at $\mathrm{z}=\mathrm{z}_{\mathrm{b}}$. Trajectories passing through the object point intersect at an image point are independent of there initial slope $\left(\mathrm{x}_{\mathrm{a}}^{\prime}, \mathrm{y}_{\mathrm{a}}^{\prime}\right)$. Thus all Gaussian trajectories emitted from an object point $\mathrm{P}_{\mathrm{a}}\left(\mathrm{x}_{\mathrm{a}}, \mathrm{y}_{\mathrm{a}}\right)$ in the object plane, regardless of their slope, are focused at a point image $\mathrm{P}_{\mathrm{b}}\left(\mathrm{x}_{\mathrm{b}}, \mathrm{y}_{\mathrm{b}}\right)$ in the image plane $\mathrm{z}=\mathrm{z}_{\mathrm{b}}$.

\subsection{Aberration theory}

Rotationally symmetric electric fields may be generated by systems of metallic electrodes that are rotationally symmetrical in geometry and configuration and between which appropriate voltages are applied. In these electric fields, the equal-potential surfaces are rotationally symmetrical curved surfaces, which can be made to focus ion beams. 


\subsubsection{Spherical aberration}

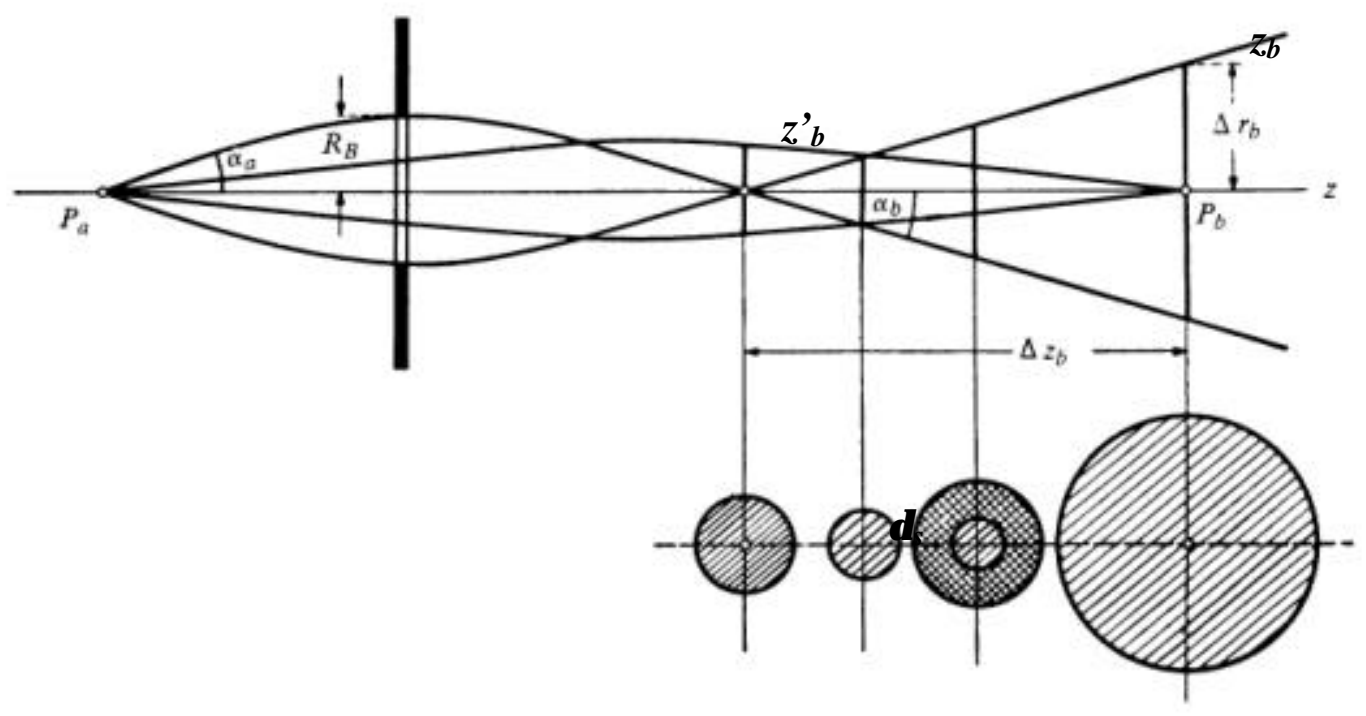

Figure 4.2 Spherical aberration. ${ }^{2}$

The spherical aberration stems from a basic property of rotationally symmetrical fields. As shown in Figure 4.2, the focusing action of the fields in the region farther from the axis is stronger than that in the region nearer to the axis. The image position $\mathrm{z}^{\prime}{ }_{\mathrm{b}}$ for the marginal off-axis trajectories will be in front of the Gaussian focus and further from the Gaussian image plane than the position $\mathrm{z}_{\mathrm{b}}$ for paraxial trajectories. The minimum radius of the beam $\delta_{s}$, which is used to characterize spherical aberration, proportional to the third power of the angular aperture $\alpha_{a}$ in the object plane.

In electron or ion optical imaging devices, the ultimate resolution is limited mainly by the spherical aberration which is the only nonzero geometrical aberration at the axis and therefore the most harmful. It can be decreased by reduction of $\alpha_{a}$. 


\subsubsection{Coma aberration}

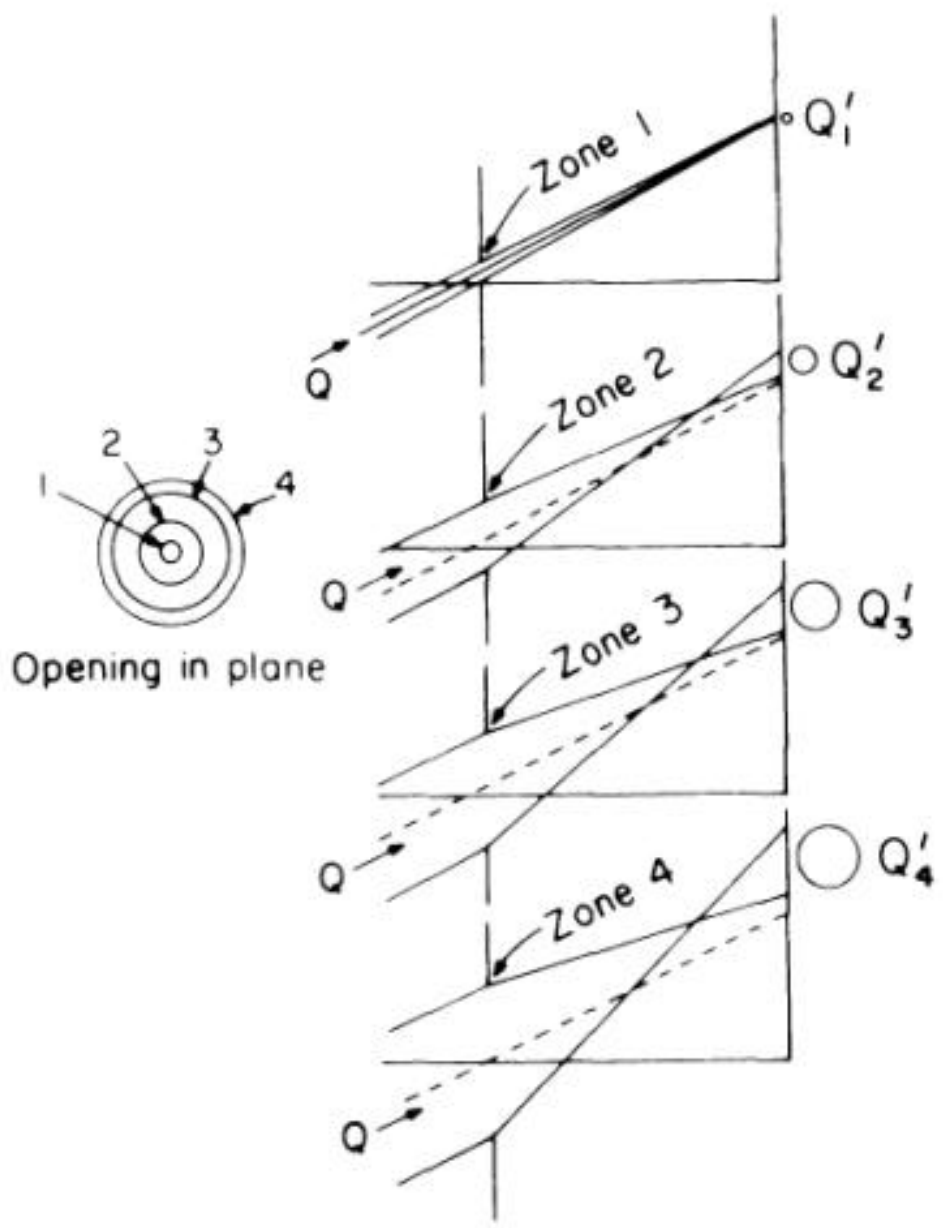

Figure 4.3 Coma aberration. ${ }^{2}$

As shown in Fig 4.3, the coma originates from the following fact. In a conical electron beam, a series of rays with varying angular aperture will pass those different zones of the lens which correspond to different focal lengths and will form a series of circles with different radius. Coma is proportional both to the first power of the height $x_{a}$ in the object pane and the second power of the contour radius $r_{b}$ in the aperture plane. 


\subsubsection{Field curvature and astigmatism}

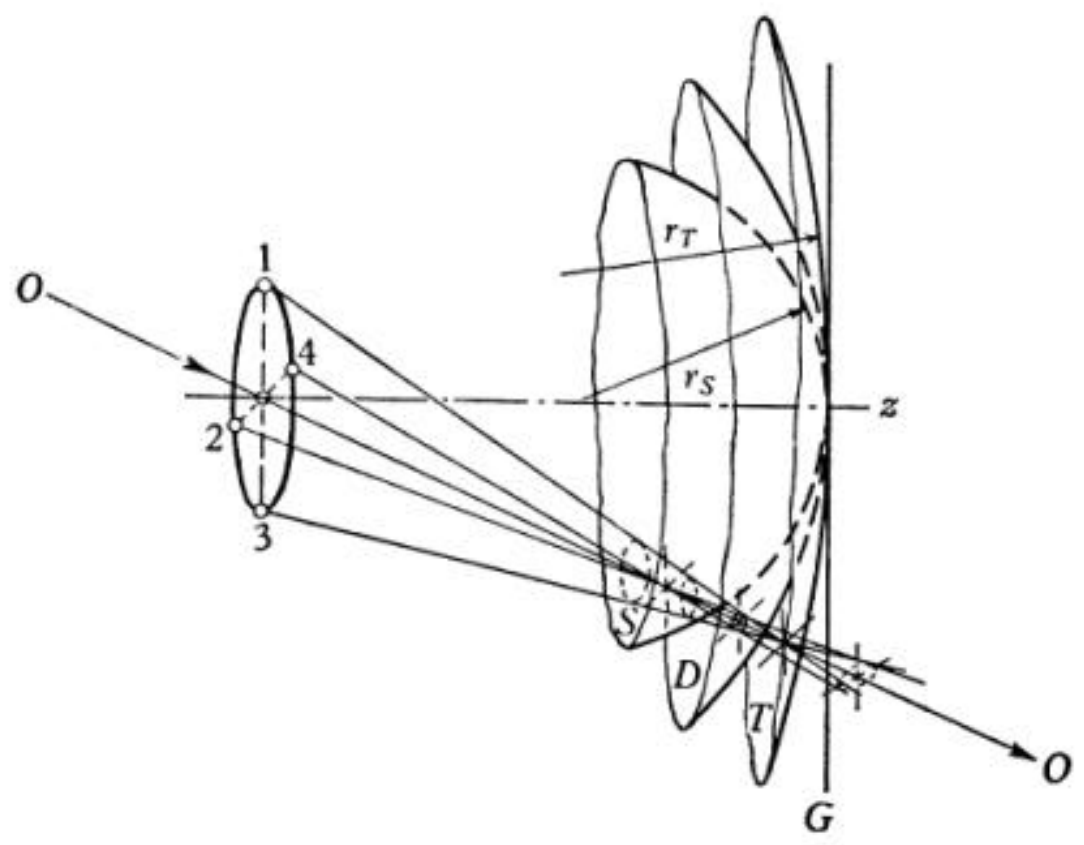

Figure 4.4 Field curvature and astigmatism. ${ }^{2}$

First, the ions from different points with varying heights $x_{a}$ in the object plane will travel through different parts of the field. Since the focusing action of the field in the region further from the axis will be stronger than that in the region nearer to the axis, the object point with larger height $\mathrm{x}_{\mathrm{a}}$ will be convergent to an image point farther from the Gaussian image plane than that object point with smaller height surface, resulting in a concave image towards the object (Fig. 4.4). Secondly, in the off-axis region, a conical beam will experience different focusing action: along the radial direction passing through the object, and along the perpendicular direction. Therefore, the ion beam under two different convergent conditions will form two separate image surfaces, which are known as astigmatism. Field curvature and astigmatism are proportional to both the second power of the height $x_{a}$ in the object plane and the first power of the contour radius $\mathbb{b}_{b}$ in the aperture plane. 


\subsubsection{Distortion}

Distortion is proportional to the third order of the height $x_{\mathrm{a}}$ in the object plane, but is independent of the contour radius $r_{B}$ in the aperture plane.

\subsubsection{Chromatic aberration}

Chromatic aberration in light optics arises from the fact that light of different color (wavelength) is refracted with different strength. In ion optics, the ions passing through a lens with different energy will be refracted differently. First-order chromatic aberration is proportional to the fractional energy spread, $\Delta \mathrm{V} / \mathrm{V}$, at the image plane, and to the first power of $\alpha$ or $r$.

In rotationally symmetric electron lenses, chromatic aberration cannot be completely eliminated, but it can be reduced by increasing the acceleration voltage, by reducing the fluctuations in the accelerating voltage and by limiting the aperture.

In summary, geometrical aberrations have a functional dependence of the form $\alpha^{\mathrm{i}} \mathrm{r}^{\mathrm{j}}$, where $\alpha=$ beam half angle at the image plane, $r=$ off-axis distance at the image plane, First-order chromatic aberration are proportional to the fractional energy spread, $\Delta \mathrm{V} / \mathrm{V}$, at the image plane, and to the first power of $\alpha$ or r. Table 4.1 shows the general form of the primary aberrations for a shaped beam electron focusing and deflection system. In this table, $\mathrm{D}$ is the deflection distance and $\mathrm{B}$ the shaped beam size at the image plane. The five aberrations which can be eliminated by dynamic correction are shown in bold type in Table 4.1. ${ }^{3}$ 
Table 4.1 General form of the Primary aberrations of a shaped beam focusing and deflection system. (The terms which can be reduced or eliminated by dynamic corrections are shown in bold type.)

\begin{tabular}{lcccc}
\hline Aberrations & Axial & Deflection & Shaped Beam & Hybrid \\
\hline Spherical & $\mathrm{C}_{\mathrm{s}} \alpha^{3}$ & - & - & - \\
Aberrations & & & & \\
Coma & - & $\mathrm{M}_{\mathrm{co}} \alpha^{2} \mathrm{D}$ & $\mathrm{S}_{\mathrm{co}} \alpha^{2} \mathrm{~B}$ & - \\
Field Curvature & - & $\mathbf{M}_{\mathrm{fc}} \alpha \mathbf{D}^{2}$ & $\mathrm{~S}_{\mathrm{fc}} \alpha \mathrm{B}^{2}$ & $\mathrm{H}_{\mathrm{fc}} \alpha \mathrm{DB}$ \\
Astigmatism & - & $\mathbf{M}_{\mathrm{as}} \alpha \mathbf{D}^{2}$ & $\mathrm{~S}_{\mathrm{as}} \alpha \mathrm{B}^{2}$ & $\mathrm{H}_{\mathrm{as}} \alpha \mathrm{DB}$ \\
Distortions & - & $\mathbf{M}_{\mathrm{di}} \mathbf{D}^{3}$ & $\mathrm{~S}_{\mathrm{di}} \mathrm{B}^{3}$ & $\left(\mathbf{H}_{\mathrm{di1} 1}+\mathbf{H}_{\mathrm{di} 2}\right) \mathbf{D}^{2} \mathbf{B}+$ \\
& & & & $\left(\mathrm{H}_{\mathrm{di} 3}+\mathrm{H}_{\mathrm{di} 4}\right) \mathrm{DB}^{2}$ \\
Chromatic & $\mathrm{C}_{\mathrm{c}} \alpha \Delta \mathrm{V} / \mathrm{V}$ & $\mathrm{M}_{\mathrm{tc}} \mathrm{D} \Delta \mathrm{V} / \mathrm{V}$ & $\mathrm{M}_{\mathrm{tc}} \mathrm{B} \Delta \mathrm{V} / \mathrm{V}$ & - \\
Aberrations & & & & \\
\hline
\end{tabular}

\subsection{Simulation software}

Two software packages have been used to design focusing lenses for the maskless, resistless ion beam lithography system. One is IGUN, and the other is Munro's code.

\subsubsection{IGUN}

IGUN is a program for the simulation of positive ion extraction from plasma and performs ray tracing on the extracted ion beams with space charge consideration. ${ }^{4,}{ }^{5}$ It is based on the well known program EGUN for the calculation of electron and ion trajectories in electron guns and lenses. ${ }^{6}$ In general, the simulation of ion-beam formation comprises four well distinguishable parts: the numerical stable simulation of the nonlinear plasma sheath; the solution of Poisson's equation for the potential map using finite difference method; the integration of ion trajectories under the influence of fields derived from the map; and the allocation of space charge to the nodes of the map. 
IGUN takes into account of gobal space charge effect. For the last couple of iteration cycles, the space charges are averaged and recalculate the potential distribution. Ion temperature effect is added to the simulation by manually adding the beam transverse angle to the beam trajectories ${ }^{7}$, as shown in Figure 4.5. Assuming the incident ion energy is $\mathrm{E}_{0}$, and the ion temperature is $\mathrm{T}_{\mathrm{i}}$, the new transverse angle $\alpha$ can be calculated using the following formula:

$$
\left.\alpha\right|_{T_{i} \neq 0}=\left.\alpha\right|_{T_{i}=0}+\tan ^{-1}\left(\sqrt{\frac{T_{i}}{E_{0}}}\right)
$$

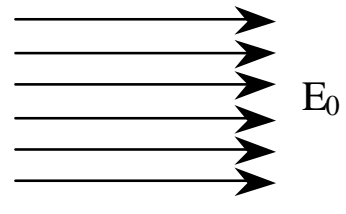

$\underline{\mathrm{T}_{\mathrm{i}}=0}$

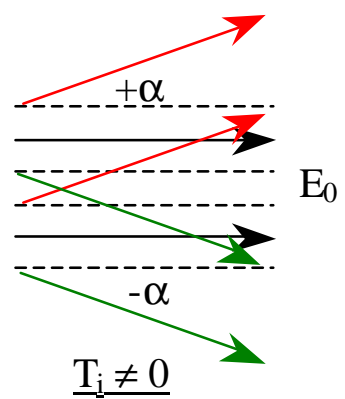

$\underline{\mathrm{T}_{\mathrm{i}} \neq \underline{0}}$

Figure 4.5 Adding ion temperature effect to IGUN simulation.

\subsubsection{Munro Code}

OPTICS and SOFEM (second order finite element method) are two of the computer software packages provided by MEBS Ltd. (England). They can be used to verify and optimize the optics performance of the focusing lens designed in IGUN.

OPTICS includes programs that can calculate axial potential distribution and field distributions in magnetic and electrostatic lenses and deflectors in electron beam focusing and deflection systems. It also computes the optical properties and aberrations up to the third-order of any combination of electron lenses and multipole deflectors. ${ }^{8}$ The first- 
order optical properties such as electrostatic lens voltages, magnification, deflection sensitivities and directions for main, sub and tertiary field deflections, are computed by numerical solution of the paraxial ray function. The third-order geometrical aberrations and first-order chromatic aberrations are computed by evaluating appropriate aberration integrals involving the paraxial rays.

SOFEM computes the potential distributions of the electrostatic lenses using the Second-Order Finite Element Method. The field computation programs in SOFEM enable the lens and deflection field functions to be computed to a much greater accuracy than those supplied with OPTICS package. SOFEM package also includes another direct ray-tracing program SORAY which can be used to compute electron trajectories through the lenses using a fourth-order Runge-Kutta method. ${ }^{8}$

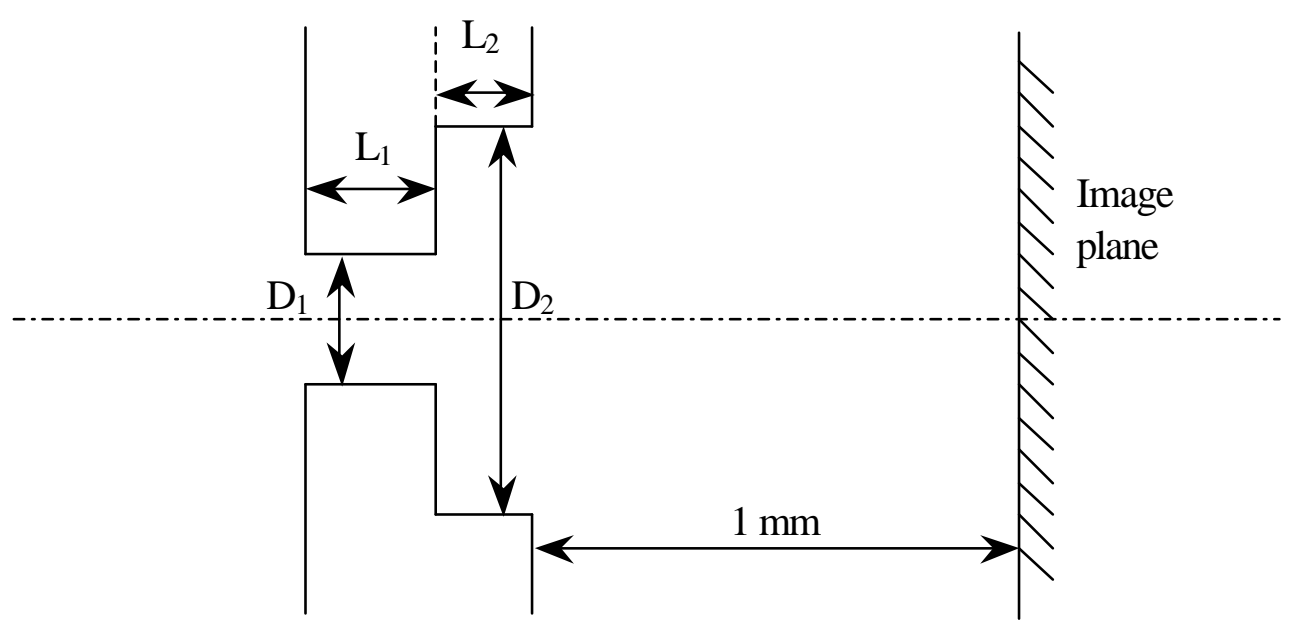

Figure 4.6 Geometry parameters for optimizing counterbored extraction apertures. 


\subsection{Single lens system}

In the beam brightness measurement discussed in Chapter 3, single lens system is used. The geometry of the first extraction aperture needs to be optimized in order to minimizing aberrations. Figure 4.6 shows the geometry parameter of a counterbored aperture. The aspect ration of the beam inlet is fixed at 5, i.e., $\mathrm{L}_{4} / \mathrm{D}_{1}=5$. The image plane is set at $1 \mathrm{~mm}$ away from the aperture. Simulations with different $L_{2}$, and $\mathrm{D}_{2}$ are performed for $\mathrm{D}_{1}=50 \mu \mathrm{m}, \mathrm{L}_{1}=250 \mu \mathrm{m}$.

Figure 4.7 shows the relationship between aberration and the diameter of the counterbored aperture and the thickness of the step. For an extraction aperture of $50 \mu \mathrm{m}$, the spherical aperture, coma, field curvature, astigmatism, distortion, and chromatic aberration decrease when the diameter of the opening aperture and reaches its minimum value when opening aperture is $300 \mu \mathrm{m}$. The aberration didn't change significantly when the thickness of the second aperture varies. Overall, for an extraction aperture of $50 \mu \mathrm{m}$, the optimum value of the counter-bored geometry is $\mathrm{D}_{2}=300 \mu \mathrm{m}$, and $\mathrm{L}_{2}=300 \mu \mathrm{m}$, and the demagnification is $5 \times$, which indicates the Gaussian image size is around $10 \mu \mathrm{m}$. The overall blur is $4 \mu \mathrm{m}$. 


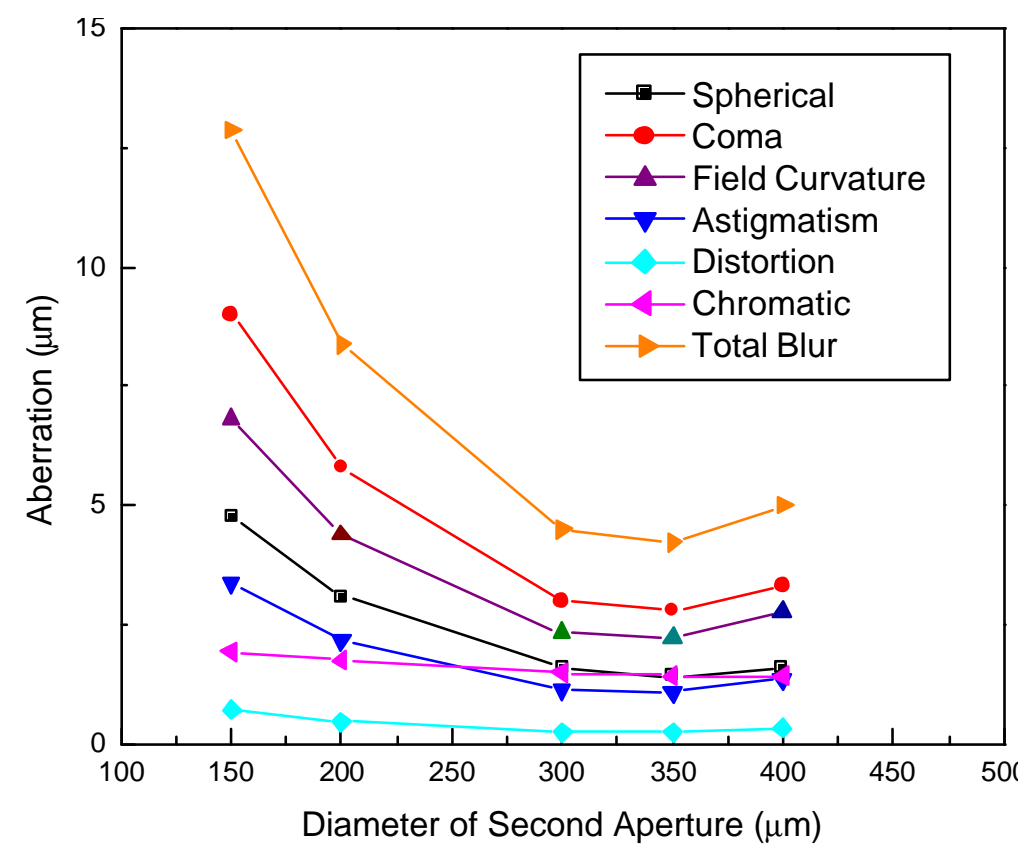

(a)

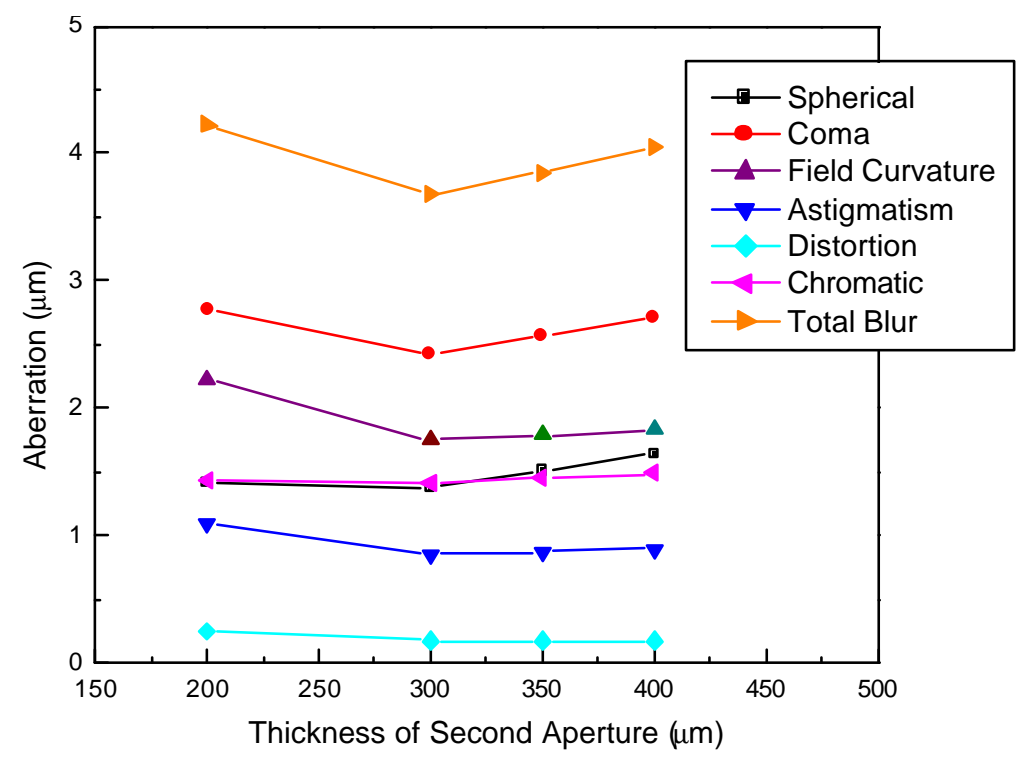

(b)

Figure 4.7 Aberration dependence on diameter of counterbored aperture $\mathrm{D}_{2}$ and thickness of the second step $\mathrm{L}_{2}$, for $\mathrm{D}_{1}=50 \mu \mathrm{m}, \mathrm{L}_{1}=250 \mu \mathrm{m}$.

\subsection{Two-lens system}

The two-lens column shown in Figure 4.8 is very compact with a total length of about $1.1 \mathrm{~cm}$. The first lens of the column serves to extract the ion beam from the plasma source. The first electrode, with an aperture of $50 \mu \mathrm{m}$ in diameter, has the same potential 
as the wall of the ion source. The second electrode is biased at $-1200 \mathrm{~V}$ relative to the first electrode. The second is an Einzel lens with three electrodes, which is for focusing the extracted beam in a decelerate-accelerate mode. The middle electrode of the Einzel lens can be split into to quadrapole electrostatic deflector to achieve beam steering in both $\mathrm{x}$ and $\mathrm{y}$ directions.

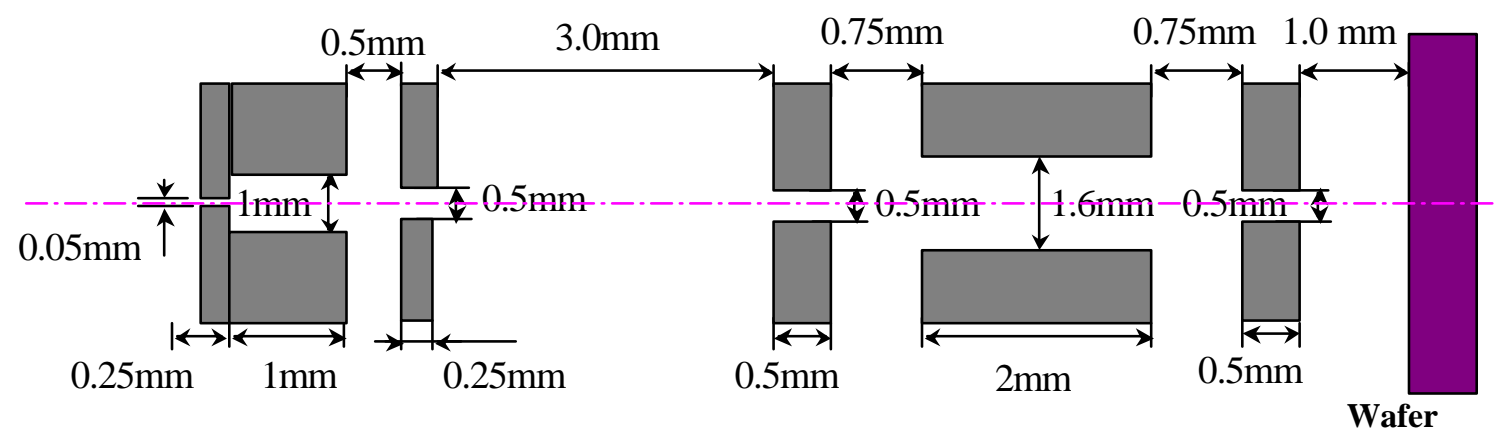

Figure 4.8 Design of a two-lens column for MRIBL system. (b) Ray tracing simulations using Monro's code.

\subsubsection{IGUN simulation results}

Figure 4.9 (a) shows the ion beam trajectories using IGUN simulation without taking ion temperature into account. The current extracted from the ion source is $63 \mathrm{nA}$. According to the beam distribution at the focal plane shown in Fig. 4.9 (b), the beam spot size is around $30 \mu \mathrm{m}$.

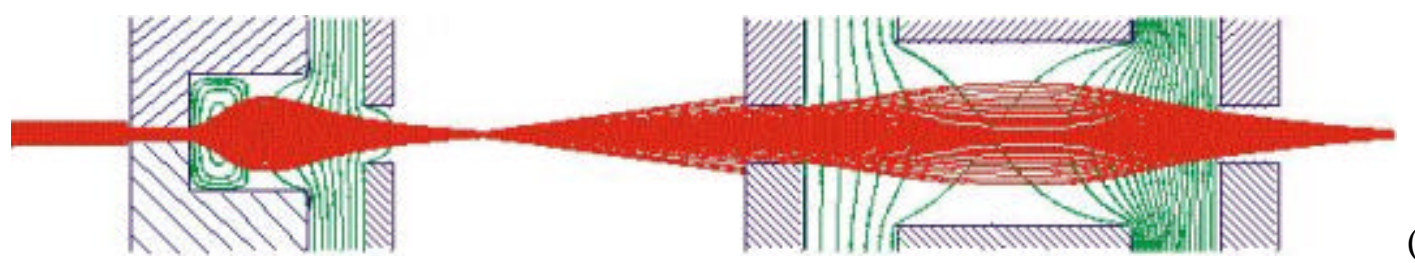

(a) 


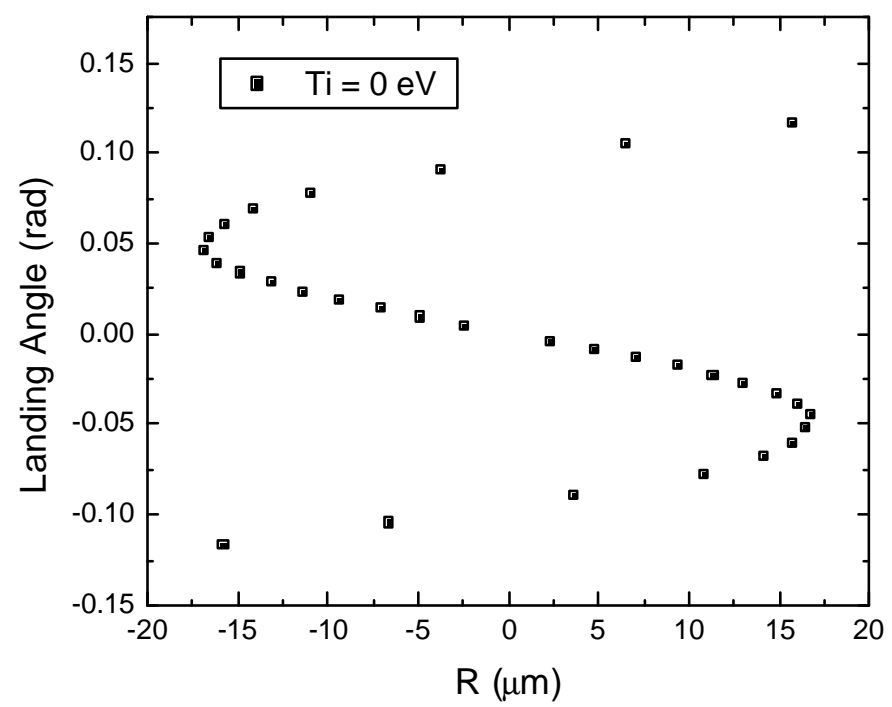

(b)

Figure 4.9 Ray tracing simulation results using IGUN when $T_{i}=0$. (a) Ion beam trajectories. (b) Ion distribution at the focal plane.

As stated in Section 4.3.1, ion temperature effect can be added into IGUN simulation. Assuming the ion temperature is $0.1 \mathrm{eV}$, the transverse half angle $\alpha$ is approximately 44 mrad for the beam at energy of $50 \mathrm{eV}$. The simulation result with ion temperature effect is shown in Fig. 4.10. Large transverse angle leads to more aberration. Himinating those large angle beamlets shown in Fig. 4.10(b), the beam size is around $40 \mu \mathrm{m}$.

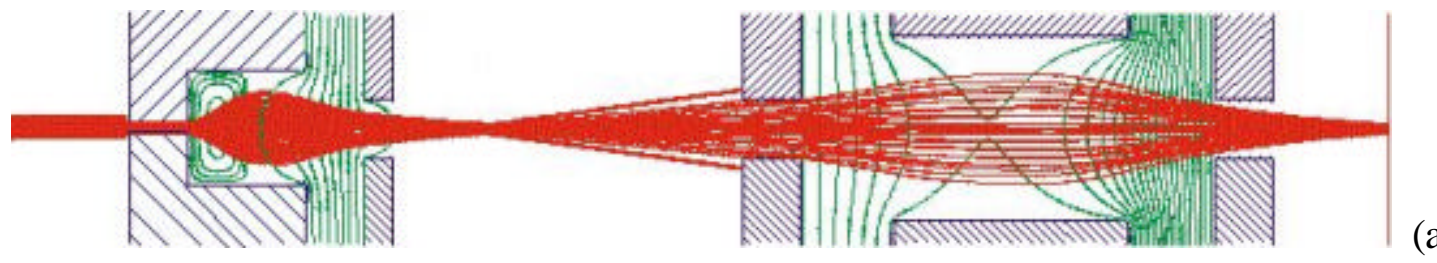

(a) 


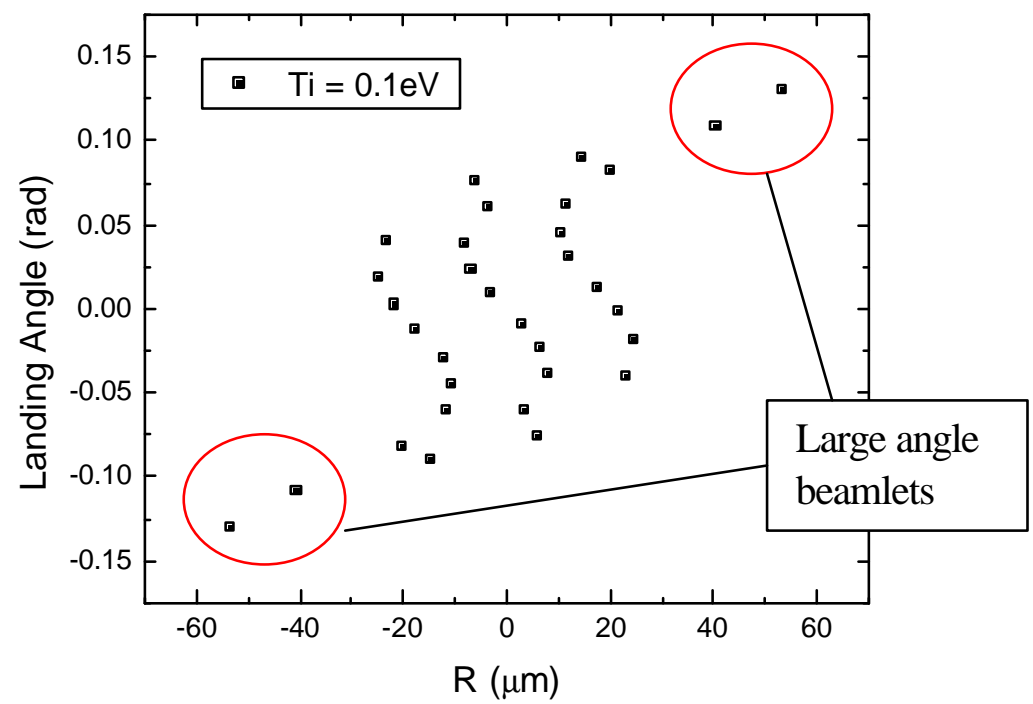

(b)

Figure 4.10 Ray tracing simulation results using IGUN when $T_{i}=0.1 \mathrm{eV}$. (a) Ion beam trajectories. (b) Ion distribution at the focal plane.

\subsubsection{Munro's code simulation results}

Using Munro's code, based on aberration theory, the demagnification of this two-lens system is $10 \times$ with object locating at $1 \mathrm{~mm}$ away in front of the first extraction aperture, and the image system locates $1 \mathrm{~mm}$ away from the last electrode. The object size used in the simulation is the diameter of the first electrode, which indicates that the Gaussian image size is $5 \mu \mathrm{m}$. However, the overall blur calculated using the ABER code is $16 \mathrm{um}$ which is far greater than the image size itself. The only way to reduce aberration is to reduce the beam half angle. Table 4.2 lists all aberration values with three different beam angle at the image plane: $75 \mathrm{mrad}, 30 \mathrm{mrad}$, and $10 \mathrm{mrad}$. When the beam angle reduces to $10 \mathrm{mrad}$, the overall blur decreases from $16 \mu \mathrm{m}$ to $1.4 \mu \mathrm{m}$. 
Table 4.2 Munro's simulation results: aberration and beam blur for different beam angle at the image plane.

\begin{tabular}{|l|c|c|c|}
\hline angle $(\mathrm{mrad})$ & 75.00 & 30.00 & 10.00 \\
\hline Spherical $(\mu \mathrm{m})$ & 6.86 & 0.44 & 0.02 \\
\hline Coma $(\mu \mathrm{m})$ & 12.04 & 1.94 & 0.22 \\
\hline Astigmatism $(\mu \mathrm{m})$ & 8.48 & 3.40 & 1.13 \\
\hline Field curvature $(\mu \mathrm{m})$ & 4.23 & 1.70 & 0.57 \\
\hline Distortion $(\mu \mathrm{m})$ & 0.84 & 0.84 & 0.84 \\
\hline Chromatic $(\mu \mathrm{m})$ & 1.13 & 0.56 & 0.39 \\
\hline Beam Blur $(\mu \mathrm{m})$ & 16.82 & 4.33 & 1.34 \\
\hline
\end{tabular}

Ray tracing simulation is performed using Munro's SORAY package. Unlike IGUN, SORAY assumes that parallel beams enter the column. Fig. 4.11 shows the current distribution at the image plane, given by Munro's ray tracing code. From $20 \%$ to $80 \%$, the beam spot size is $15 \mu \mathrm{m}$, which is less than IGUN predicts. One point is that IGUN simulates plasma extraction, rather than the assumption of parallel beam incident. The other point is that IGUN's results included $100 \%$ of the beam.

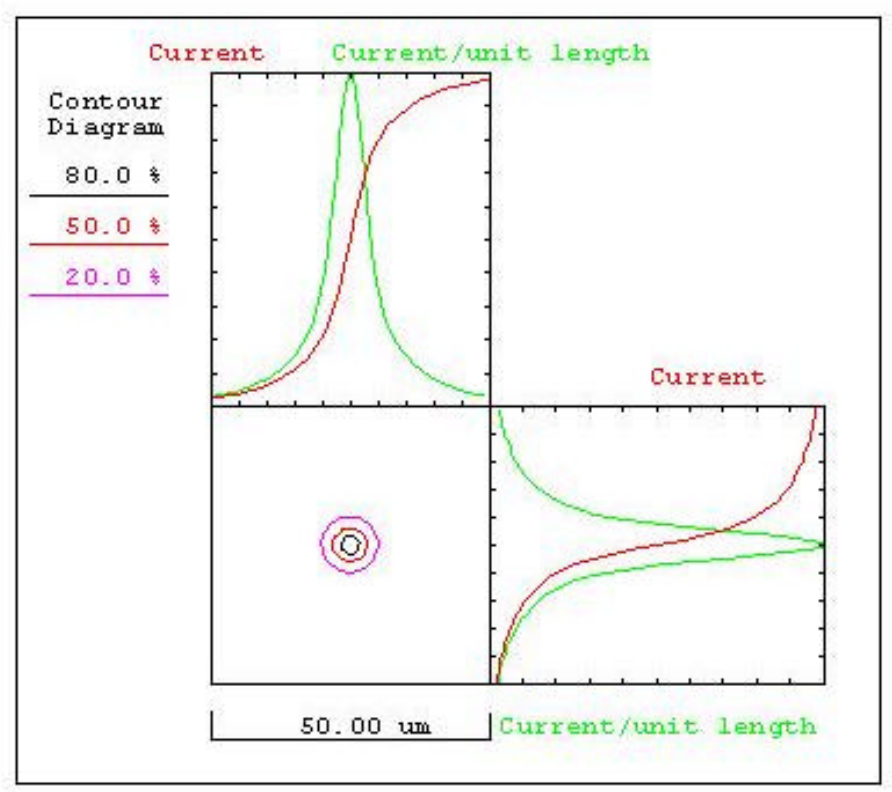

Figure 4.11 Ion distribution at the focal plane given by Munro's code. 


\subsection{Beam spot size measurement}

\subsubsection{Column fabrication}

All the electrodes are made of molybdenum to reduce sputtering. The apertures are mechanically drilled, and are aligned using a Nickon comparator. Picture of column after assembled is shown in Fig 4.12.

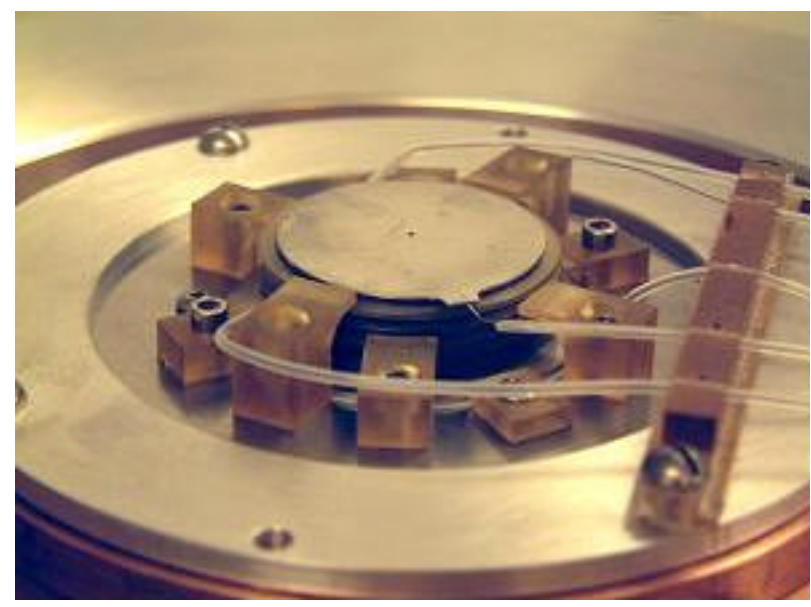

Figure 4.12 Picture of the two-lens column for MRIBL system.

\subsubsection{Experiment setup and results}

The beam spot size can be measured as shown in Fig. 4.13. It is very similar to the virtual source size measurement described in Chapter 3, except that the knife edge is located at the focal plane of the beam. According to the current distribution shown in Fig. 4.14, about $73 \mathrm{nA}$ of oxygen ions are extracted from the source and focused down to the target. The beam spot size from $20 \%$ to $80 \%$ is around 25 um. This result matches with both IGUN and Munro's simulation results very well. Beam exposure results later shown in Chapter 5 will also confirm this measurement. 


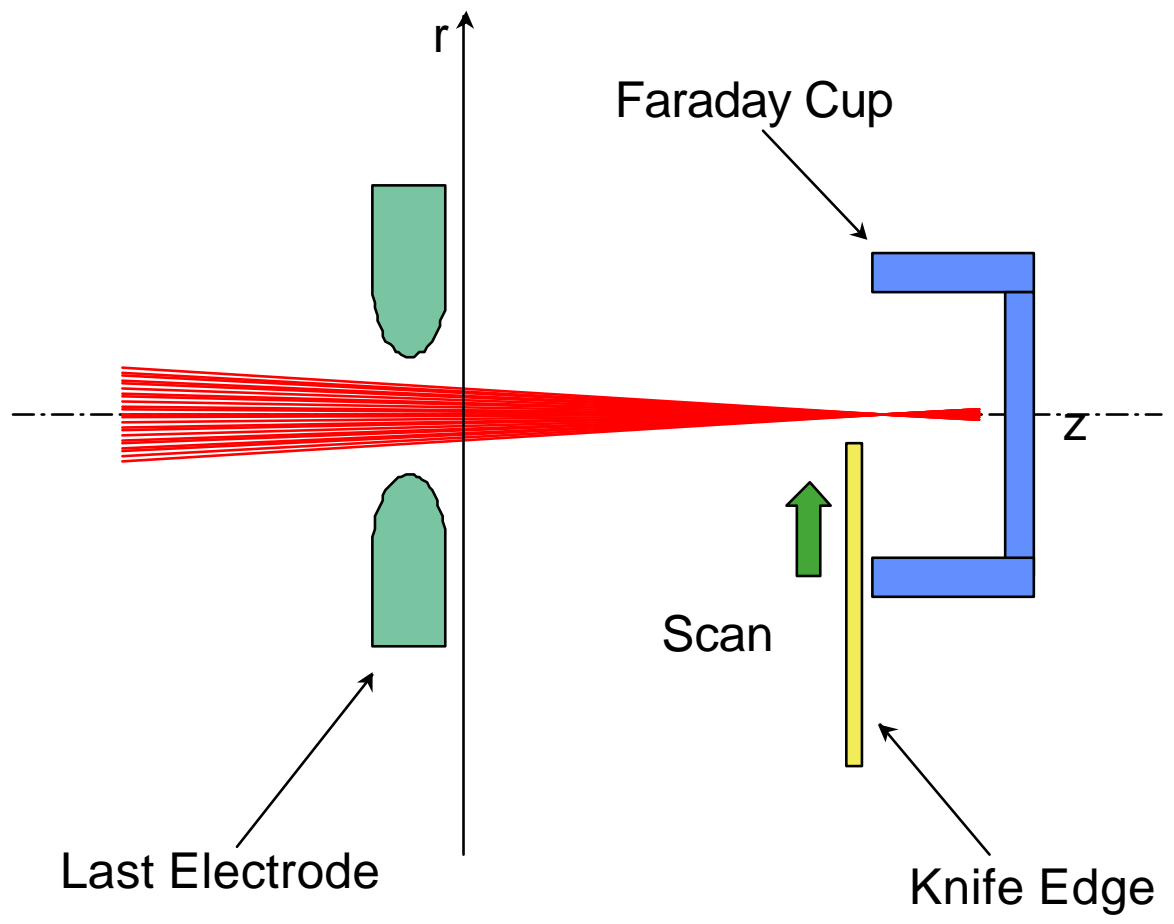

Figure 4.13 Schematic diagram of the beam spot size measurement setup.

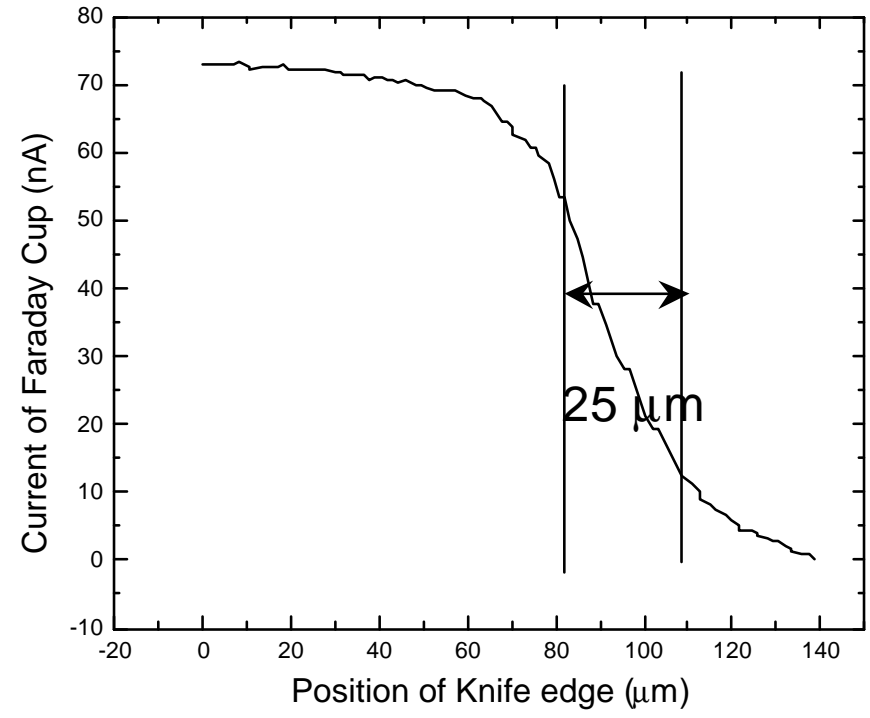

Figure 4.14 Results of the beam spot size measurement. 


\subsection{Improvement of ion optics design}

More has to be done for further improving the ion optics design to achieve smaller spot size. First, by reducing the beam angle, aberration can be decreased significantly, as discussed in Section 4.5.1. Second approach is to use a smaller extraction aperture. With the same demagnification factor, smaller image should be obtained.

\subsubsection{Adding limiting aperture}

A limiting aperture can be added between the two lenses, stopping the large angle ion beams from reaching the downstream. With an aperture of $100 \mu \mathrm{m}$ placing at the position shown in Fig. 4.15, the aberration reduces from 16 um to 2 um. However, such an approach will sacrifice beam current.

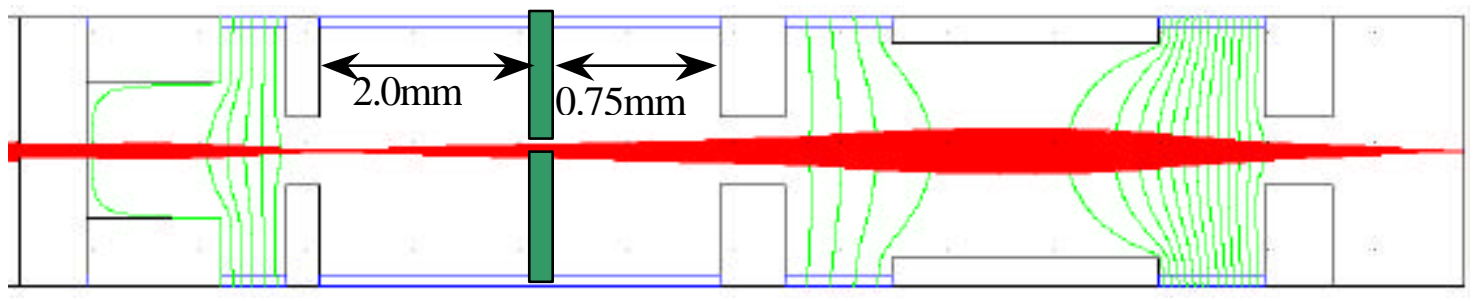

Figure 4.15 A limiting aperture to reduce aberration.

\subsubsection{Reducing size of extraction aperture}

With the same ion optics design, ten times smaller spot size could be achieved if using

a $5 \mu \mathrm{m}$ extraction aperture. Fabrication of a $5 \mu \mathrm{m}$ counterbored aperture and maintaining enough extraction current are the two challenges. 


\subsubsection{Fabrication of a $5 \mu \mathrm{m}$ counterbored aperture}

According to Munro's simulation, the optimum design for a $5 \mu \mathrm{m}$ counterbored aperture design is: $\mathrm{D}_{1}=5 \mu \mathrm{m}, \mathrm{L}_{1}=40 \mu \mathrm{m}, \mathrm{D}_{2}=40 \mu \mathrm{m}, \mathrm{L}_{2}=40 \mu \mathrm{m}$. (The definition of $\mathrm{D}_{1}, \mathrm{~L}_{1}$, $\mathrm{D}_{2}$, and $\mathrm{L}_{2}$ are the same as in Fig.4.6) Since there is a limitation for mechanical drilling, microfabrication processes are needed to fabrication such an extraction aperture. The proposed the process sequences are shown in Fig. 4.16.

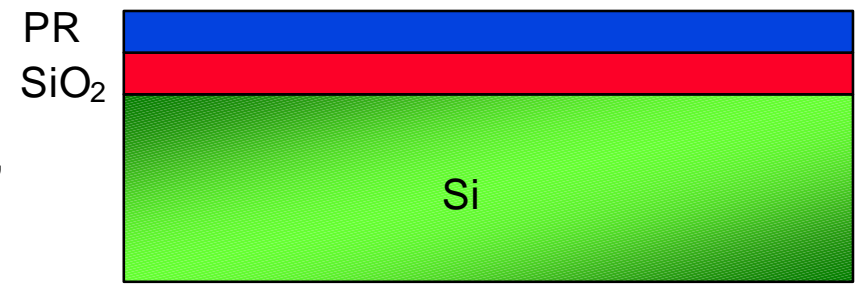

1. Start with bare silicon wafer, grow oxide,coat resist.

2. Pattern resist, etch oxide.
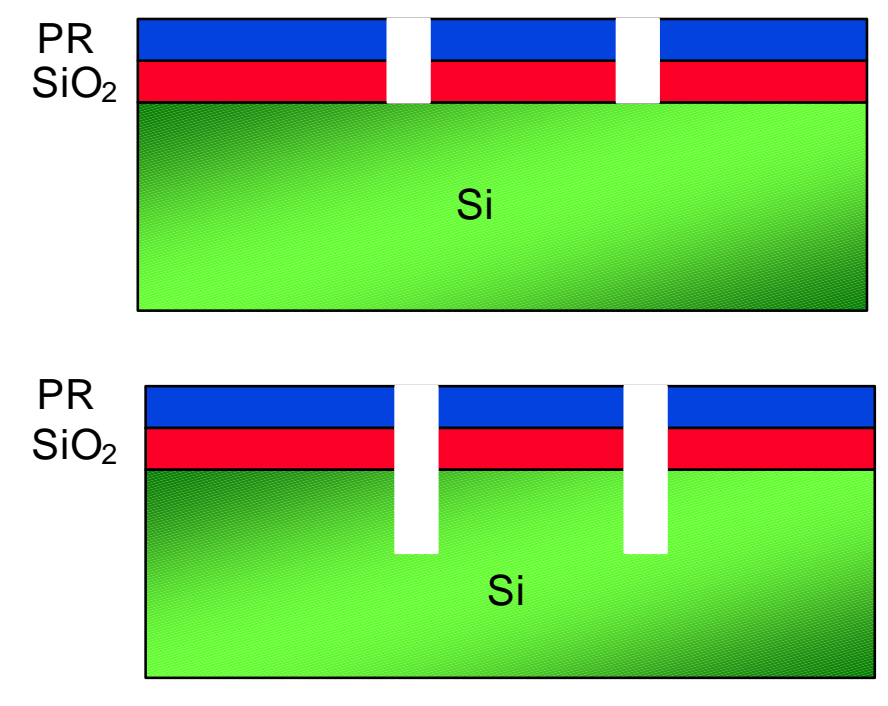

3. Deep etch silicon.

\section{HF wet etch oxide layer.}

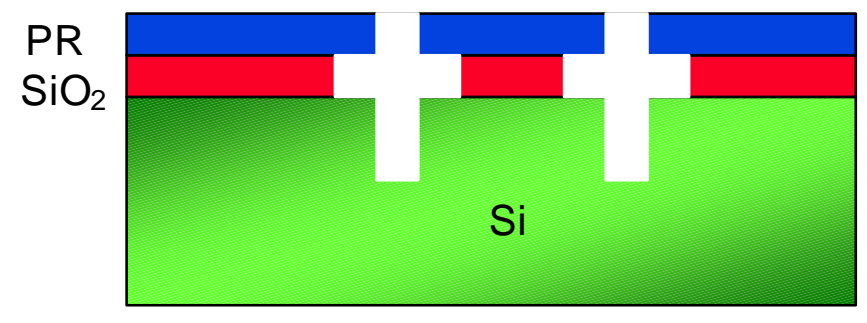


5. Strip resist.

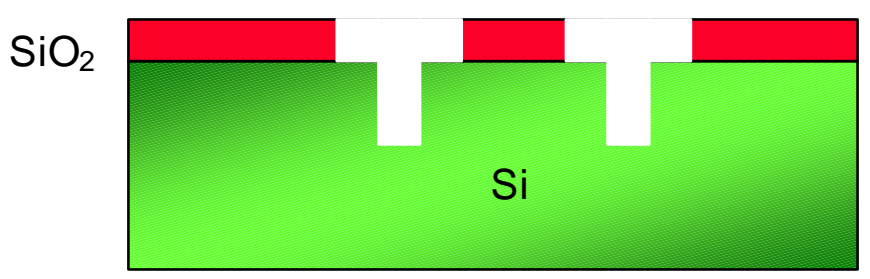

6. Deep etch silicon.

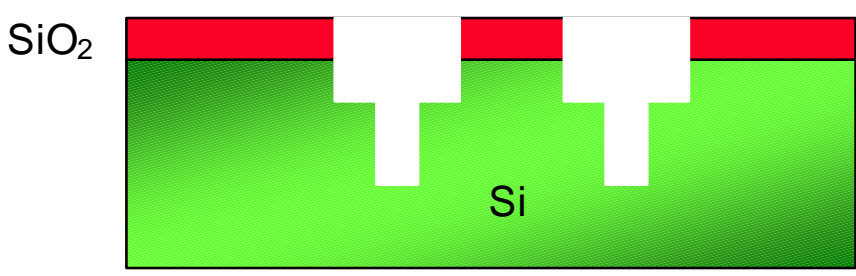

7. Etch oxide layer.

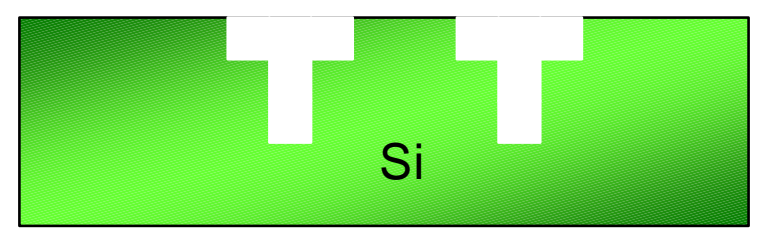

8. Deposit silicon nitride.

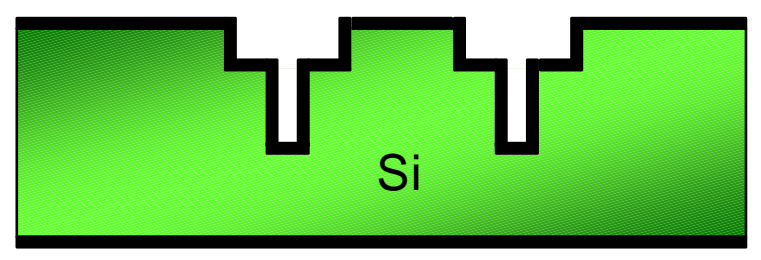

9. Pattern on the back side of the wafer.

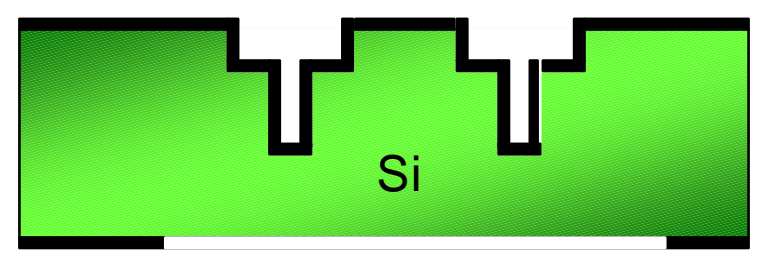

10. $\mathrm{KOH}$ etch silicon.

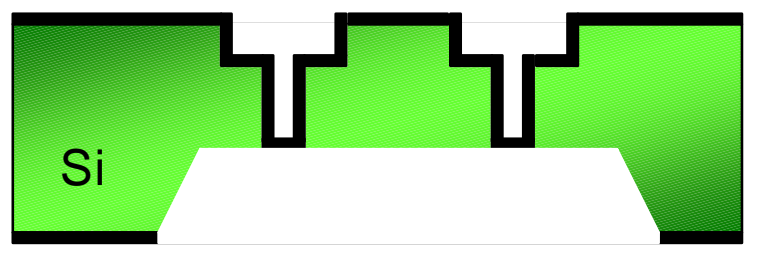


11. Strip silicon nitride layer.
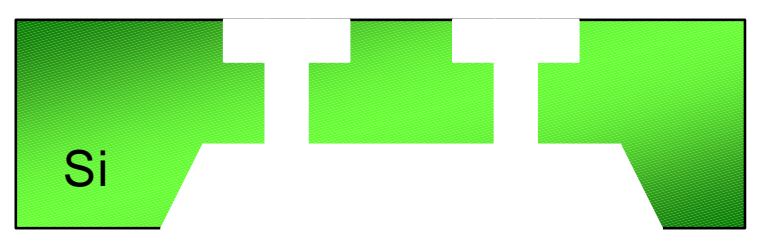

Figure 4.16 Proposed process flow for $5 \mu \mathrm{m}$ counterbored aperture fabrication.

\subsubsection{Extraction current with smaller extraction aperture}

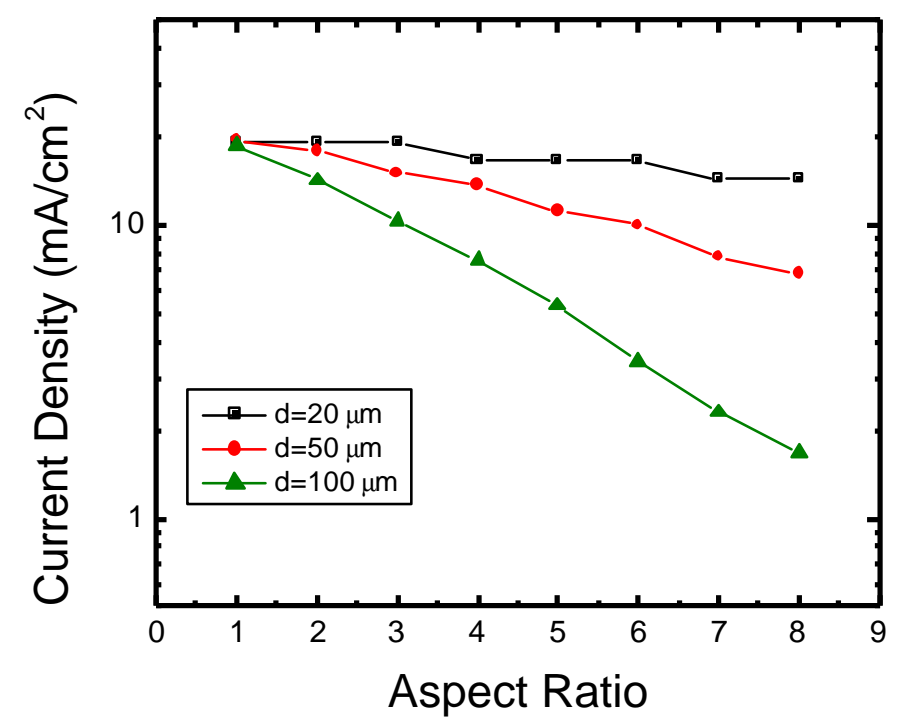

Figure 4.17 Dependence of the extracted current density on the aperture aspect ratio.

One question that may be raised is: "How much is the current that can be extracted from a smaller aperture with a high aspect ratio?" IGUN simulation indicates that actually the current density obtained from a smaller aperture is higher than that from a larger aperture if their aspect ratios are kept the same. Figure 4.17 shows the relationship 
of the current density and the aspect ratio for three different aperture sizes: $100 \mu \mathrm{m}, 50$ $\mu \mathrm{m}$, and $20 \mu \mathrm{m}$. For each aperture, the extracted current density decreases with increasing aspect ratio. However, for the same aspect ratio, (e.g. aspect ratio $=8$ ), the current density extracted from the $20 \mu \mathrm{m}$ aperture is almost ten times larger than that from the $100 \mu \mathrm{m}$ aperture.

Figure 4.18 shows the beam trajectories of the extracted beam for different size aperture with the same aspect ratio. At the source side, the equal potential line tends to bulge into the extraction aperture. Beams will be bent towards the sidewall of the channel. The longer the channel is, the more beams are lost. Therefore, the higher the aspect ratio, less beams are extracted. However, the equal potential line at the entrance to the smaller aperture is flatter than those to larger one, which results in less bending of the beam. So, fewer beams are lost.

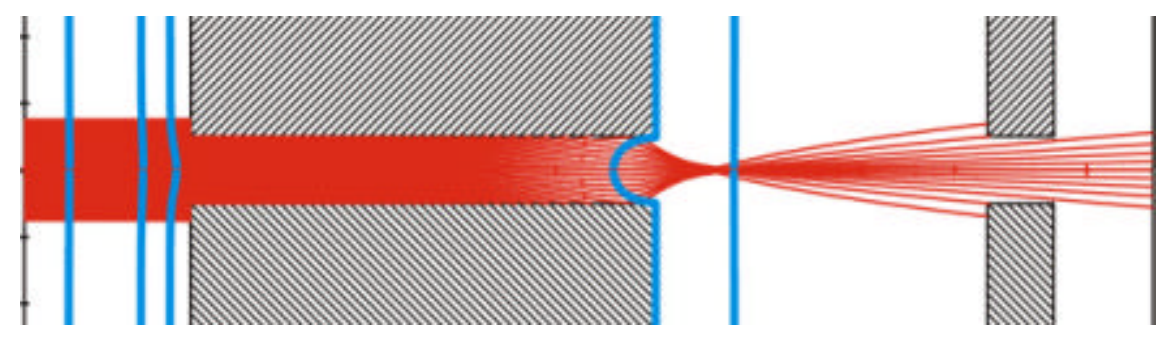

$\underline{\mathrm{D}=100 \mu \mathrm{m}}$

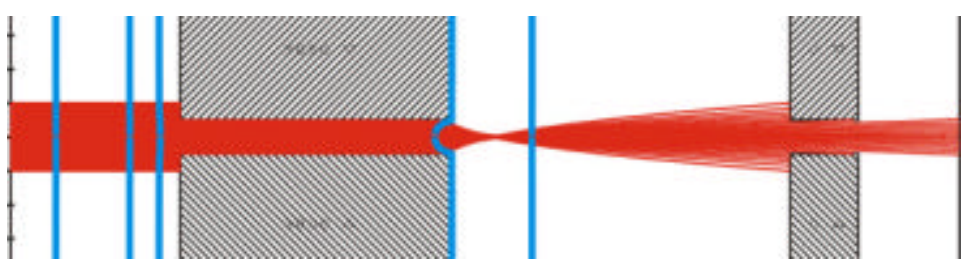

$\underline{\mathrm{D}=50 \mu \mathrm{m}}$

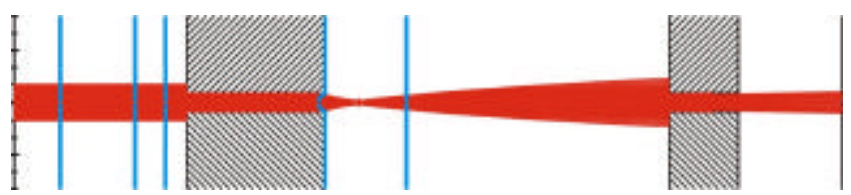

$\underline{\mathrm{D}=20 \mu \mathrm{m}}$

Figure 4.18 Beam trajectories simulated by IGUN for extraction aperture of different sizes. 
In the cases discussed above, no bias voltage is applied between the source and the first extraction aperture. If negative bias voltage is applied on the extraction aperture relative to the source, the incident ions gain some initial energy before they enters the column. Less beam bending occurs with higher initial energy. Simulations confirm that higher beam injection energy causes less beam lost in the channel, which is shown in Fig. 4.19.

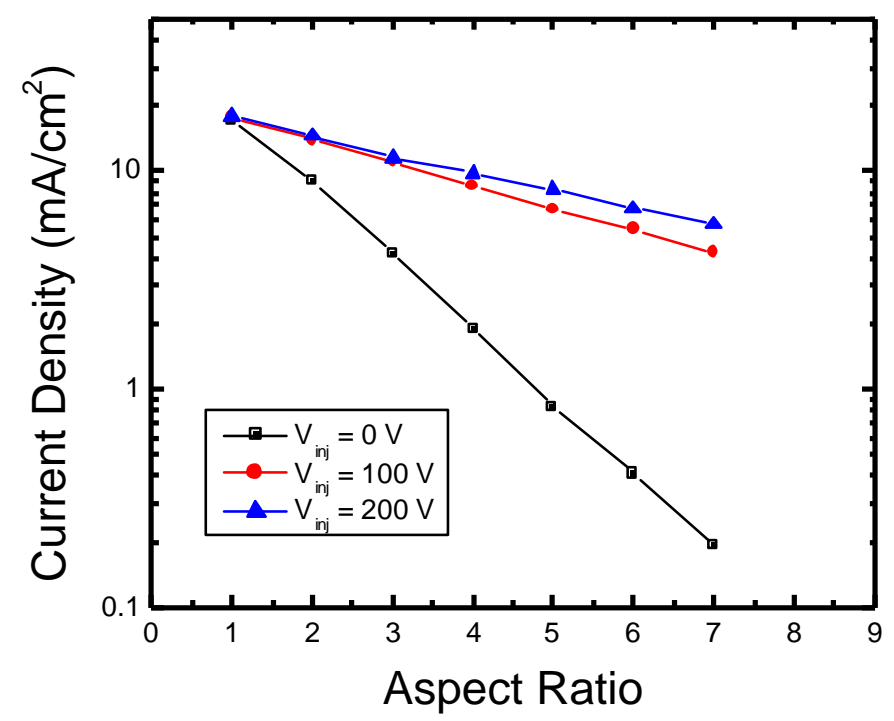

Figure 4.19 Dependence of the extracted current density on the bean injection energy.

\section{References:}

${ }^{1}$ B. Lencova, "Electrostatic Lenses", Handbook of Charged Particle Optics, edited by J. Orloff , pp.177-222, 1997.

2 "Advances in Electronics and electron physics". Edited by P. W. Hawkes. Supplements no.13. 
3 E. Munro, "Computational Techniques for Design of Charged Particle Optical Systems", in Handbook of Charged Particle Optics, edited by J. Orloff (1997).

${ }^{4}$ R. Becker, and W. B. Herrmannsfeldt, 'IGUN-a program for the simulation of positive ion extraction including magnetic fields", Rev. Sci. Instrum., 4, pp. 2756-2758, 1992.

5 R. Becker, "Numerical simulation of ion-beam formation", Rev. Sci. Instrum. 67, pp. 1132-1137, 1996.

6 W.B. Herrmannsfeldt, "EGUN-An Electron Optics and Gun Design Program", SLACReport-331, 1988.

7 J. Reijonen, M. Eardley, R. Keller, J. Kwan, K. N. Leung, D. Pickard, R. Thomae, and M. D. Williams, "Multicusp ion source for induction linac applications", Proceedings of the PAC Conference, pp1943, 1999.

${ }^{8}$ MEBS Ltd., England. “Munro’s Electron Beam Software User Manual”, 1997. 


\section{Chapter 5}

\section{Maskless, Resistless Ion Beam Lithography Processes}

\subsection{Production of various ion species}

Direct doping of a Si wafer with a focused $\mathrm{P}^{+}$or $\mathrm{BF}_{2}{ }^{+}$beam eliminates the necessity of resist which is conventionally used as a mask for ion implantation. Thin oxide formed by selectively oxidizing the poly-Si surface with a focused $\mathrm{O}_{2}^{+}$beam can be used as a hard mask for gate patterning in CMOS device process. Neither a stencil mask nor resist material is needed in such a lithography process. All these require the multicusp plasma source to produce $\mathrm{P}^{+}, \mathrm{BF}_{2}{ }^{+}$, and $\mathrm{O}_{2}{ }^{+}$beam.

The constituents of the plasma are ions and electrons, as well as un-ionized neutrals. In general, most discharges contain not only positive ions, but also negative ions. By changing the polarity of the extraction system, either positive or negative ion beams can be extracted. As shown in Fig. 5.1, Negative ion implantation or deposition reduces surface charging of the insulated substrate, because the outgoing secondary electrons 
balance the incoming negative charge of the ions. ${ }^{1}$ In FIB lithography application, the critical dimension is less deteriorated by the deflection of charged substrate, and an external source of electrons for charge neutralization is not needed. In this section, both positive and negative ions production by multicusp plasma source will be presented.

Positive lon Negative Ion

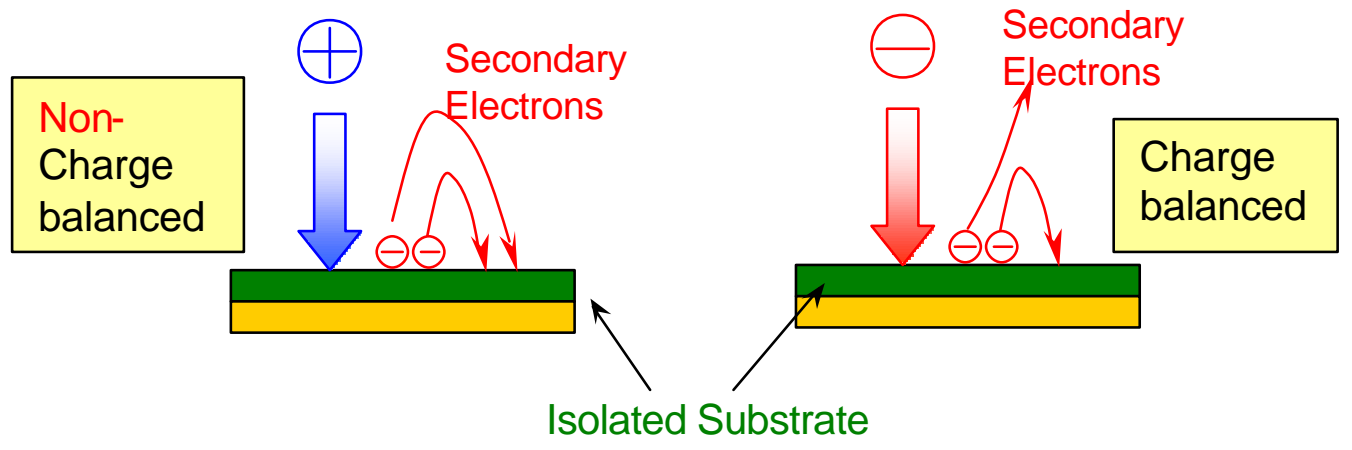

Figure 5.1 Less surface charging by using negative ions.

\subsubsection{Phosphorus ions: $P_{x}^{+}$and $P_{x}^{-}$ions $(x=1,2,3,4,5)$}

To generate phosphorus plasma, vaporization of solid phase phosphorus has been studied. A small oven as shown in Fig. 5.2 is attached to the gas inlet on the source back plate. The oven is heated up to around $425^{\circ} \mathrm{C}$ to vaporize the sample. Phosphorus vapor is introduced into the multicusp source to generate phosphorus plasma. 


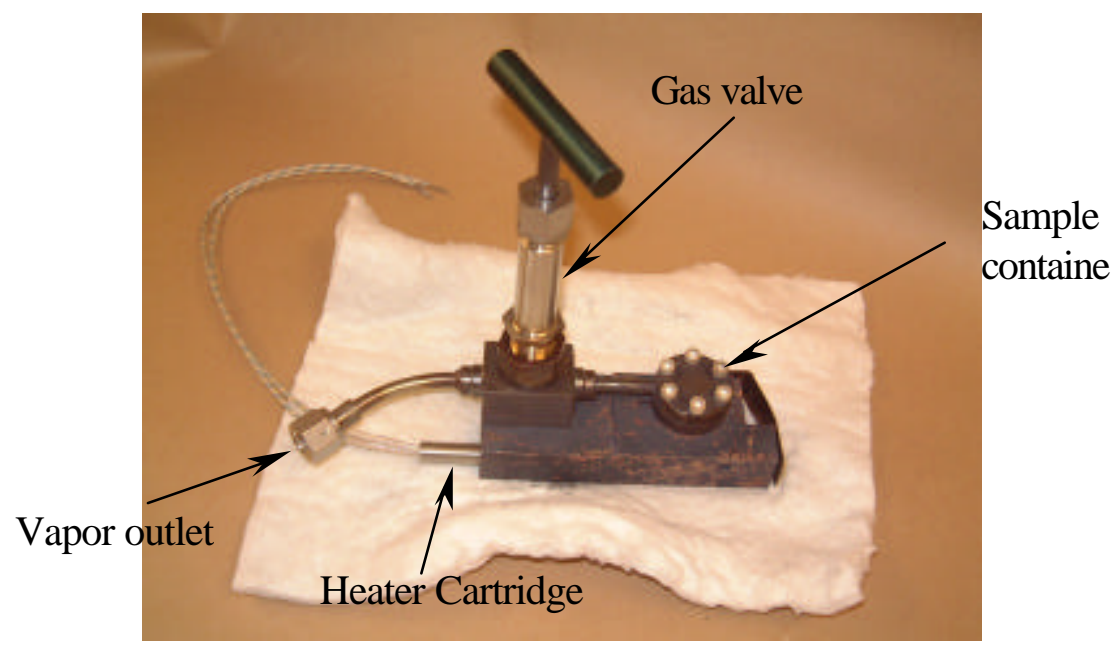

Figure 5.2 Picture of small oven used to vaporize solid phosphorus.

Figure 5.3 are the mass spectra of phosphorus plasma, for both positive and negative ions. From Fig 5.3(a), Over $90 \%$ of the positive ion species are $\mathrm{P}^{+}$. Less than $7 \%$ are $\mathrm{P}_{2}^{+}$. $\mathrm{P}_{3}{ }^{+}$and $\mathrm{P}_{4}{ }^{+}$are less than $1 \%$. On the mass spectrum, the peak of $\mathrm{Ar}^{+}$is due to the residue argon gas in the source. Argon is used as sart-up gas to warm up the source wall before phosphorus vapor is injected into the source chamber. It helps to reduce phosphorus condensation. After stable phosphorus plasma is achieved, argon gas supply will be shut off. Eventually all argon gas in the source chamber should be pumped away. As shown in Fig 5.3(b), more negative cluster phosphorus ions such as $\mathrm{P}_{2}^{-}, \mathrm{P}_{3}^{-}$etc. are produced than positive ones. 


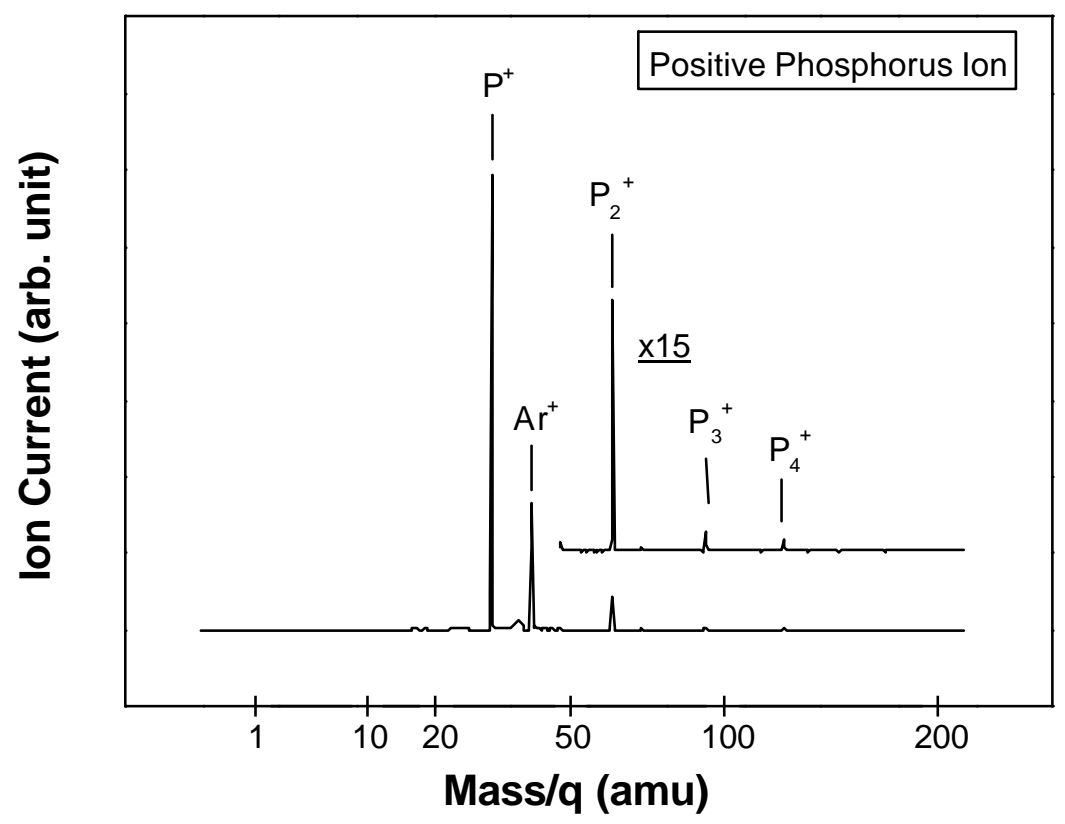

(a)

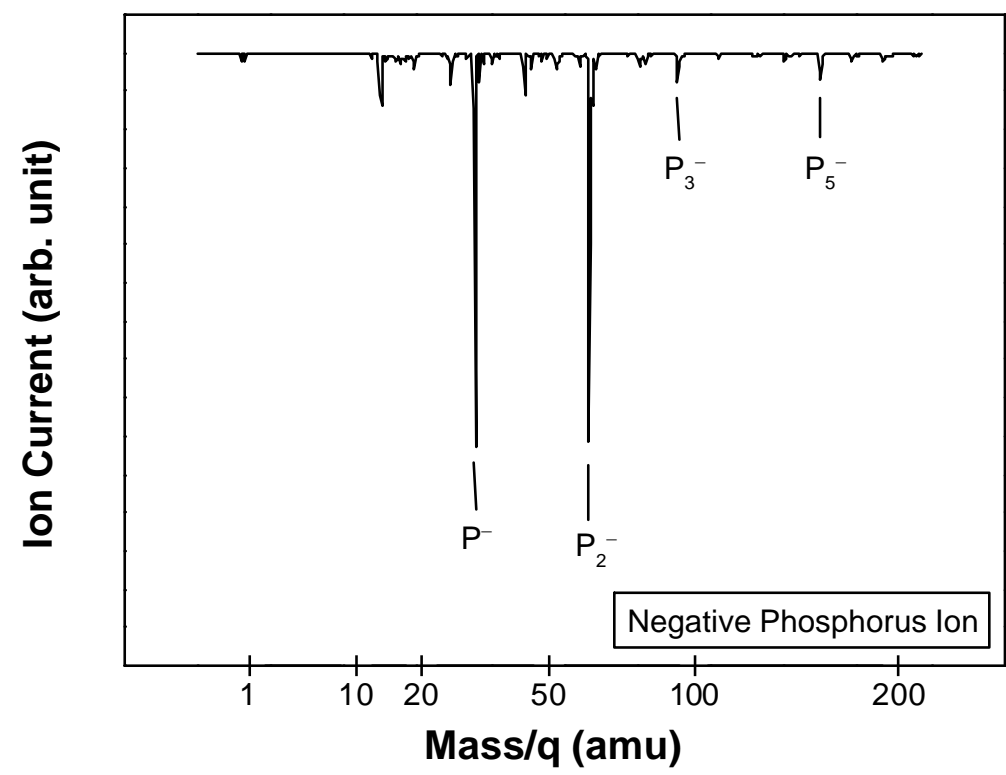

(b)

Figure 5.3 Mass spectra of Phosphorus plasma. (a) positive ions; (b) negative ions.

\subsubsection{Boron ions: $\mathrm{BF}_{\mathrm{x}}^{+}$and $\mathrm{BF}_{\mathrm{x}}^{-}$ions $(\mathrm{x}=1,2,3,4,5)$}

Figure 5.4 are the mass spectra of $\mathrm{BF}_{3}$ plasma for both positive and negative ions. For positive ions in Fig. 5.4(a), $\mathrm{B}^{+}, \mathrm{BF}^{+}, \mathrm{BF}_{2}^{+}$ions are major ion secies. Compared to $\mathrm{BF}^{+}$ and $\mathrm{BF}_{2}^{+}, \mathrm{B}^{+}$is negligible. For negative ions, higher florin content cluster ions are 
observed. This is probably due to the higher electron affinity with the present of $\mathrm{F}$. However, $\mathrm{BF}_{2}^{-}$was not found in the spectrum.

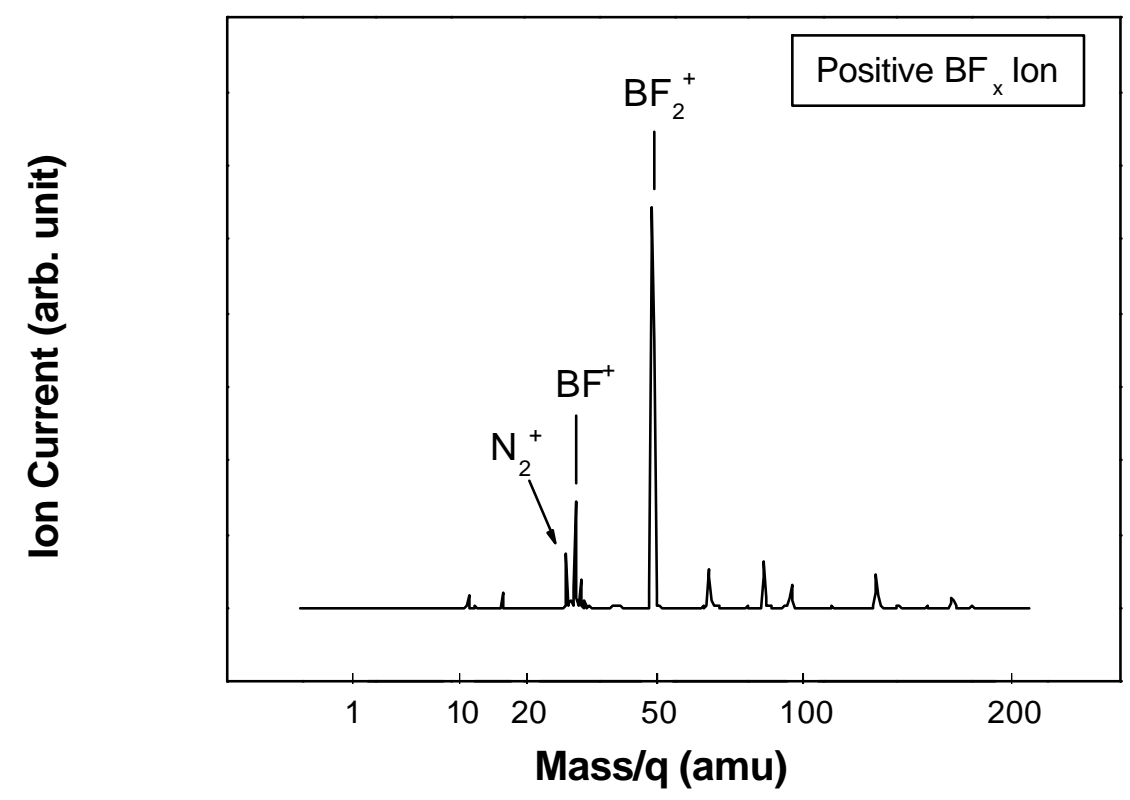

(a)

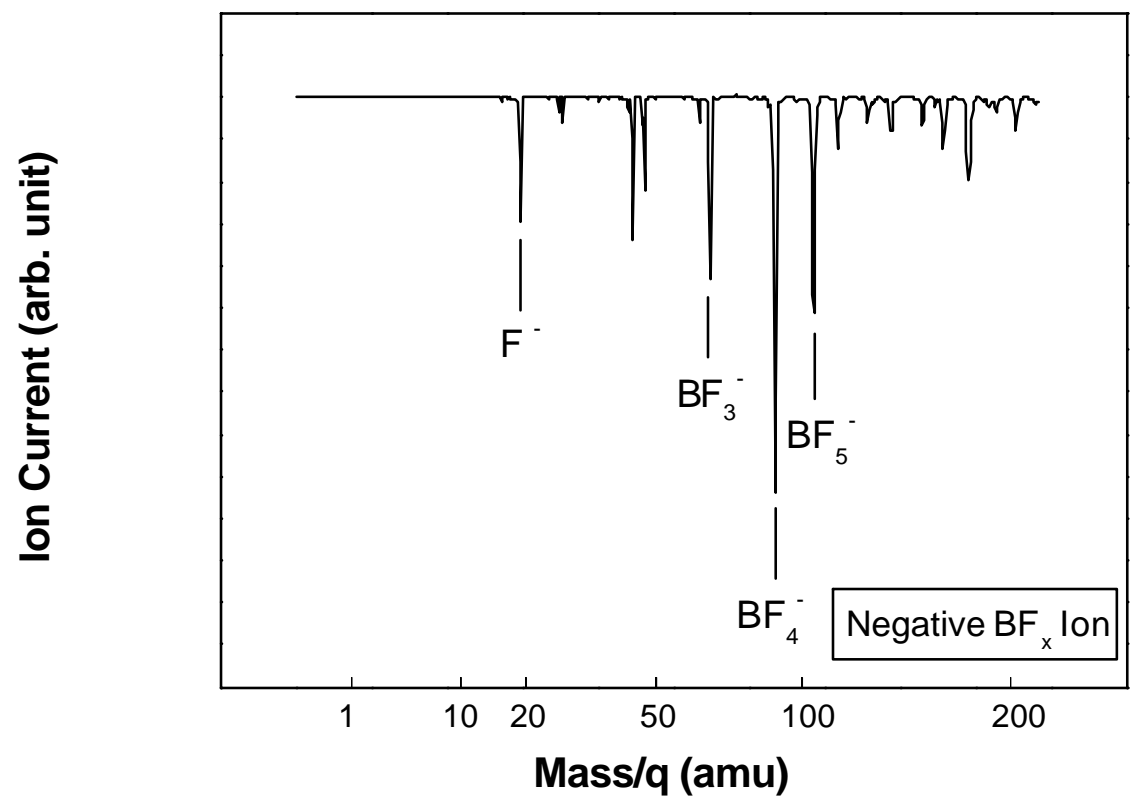

(b)

Figure 5.4 Mass spectra of $\mathrm{BF}_{3}$ plasma. (a) positive ions; (b) negative ions. 


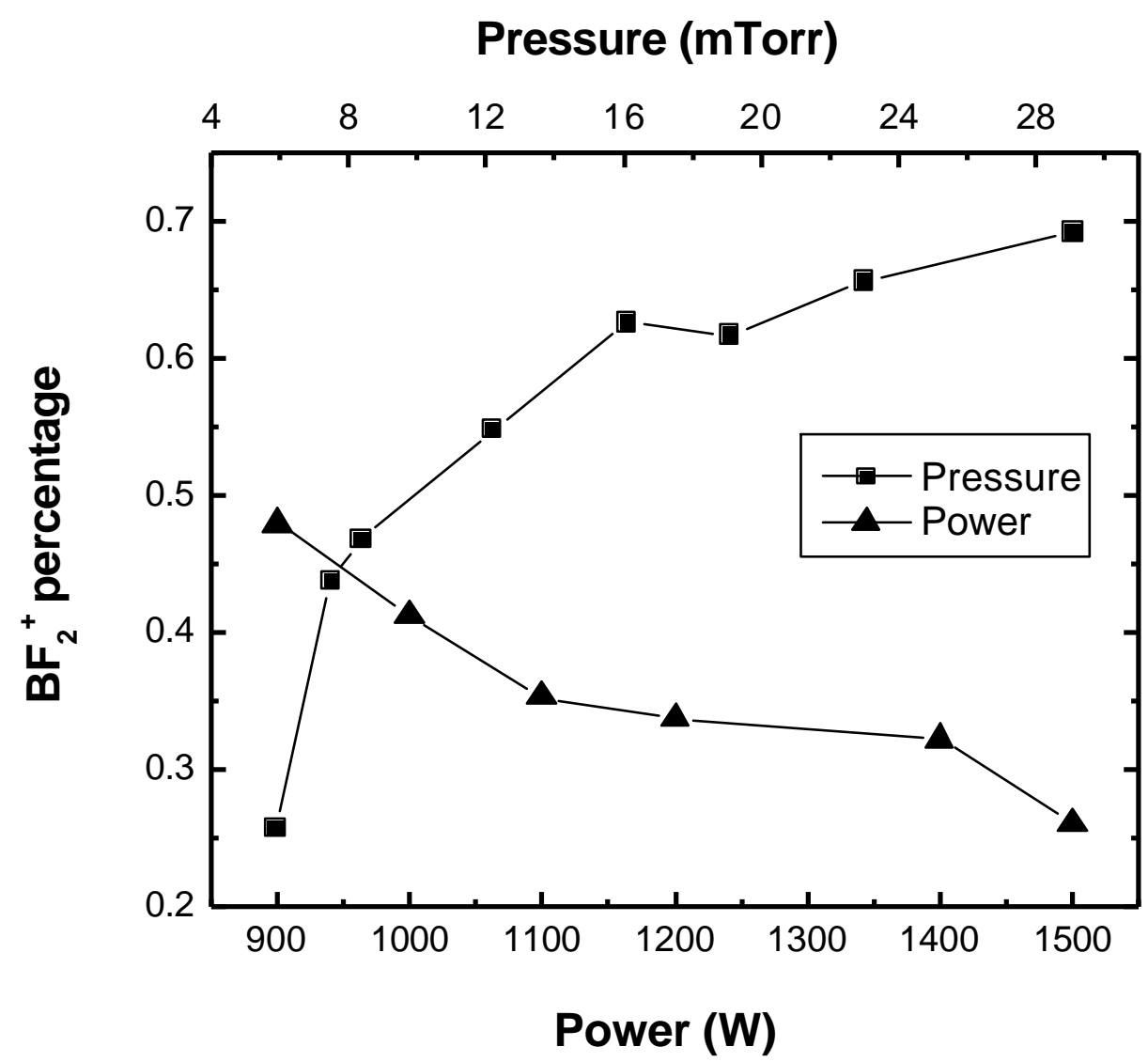

Figure 5.5 Relationship between $\mathrm{BF}_{2}{ }^{+}$percentage in $\mathrm{BF}_{3}$ plasma and gas pressure, RF power.

Focused boron ions can be selectively implanted into the $\mathrm{Si}$ substrate to form ultra shallow junction. The maximum production of single ion specie is very important to control critical dimension and the junction depth profile. Fig. 5.5 shows the relationship between $\mathrm{BF}_{2}^{+}$ion percentage and source power and $\mathrm{BF}_{3}$ gas pressure. The $\mathrm{BF}_{2}^{+}$ percentage was measured from different mass spectra at various $\mathrm{RF}$ power and $\mathrm{BF}_{3}$ gas pressure. At $1000 \mathrm{~W}$ RF power, $\mathrm{BF}_{2}{ }^{+}$ion percentage increases from $25 \%$ at 5 mTorr to almost $70 \%$ at 25 mTorr; at $6 \mathrm{mTorr}$ of $\mathrm{BF}_{3}$ gass pressure, $\mathrm{BF}_{2}{ }^{+}$ion percentage decreases 
from $50 \%$ at $900 \mathrm{~W}$ to around $25 \%$ at $1500 \mathrm{~W}$. By optimizing the gas pressure and source power, over $85 \%$ of $\mathrm{BF}_{2}{ }^{+}$ions can be achieved at 25 mTorr and $900 \mathrm{~W}$ conditions.

\subsubsection{Oxygen ions: $\mathrm{O}_{\mathrm{x}}{ }^{+}$and $\mathrm{O}_{\mathrm{x}}{ }^{-}$ions $(\mathrm{x}=1,2)$}

Figure 5.6 is the mass spectra of oxygen plasma for both positive and negative ions. For the positive ions, two ion species are produced: $\mathrm{O}^{+}$and $\mathrm{O}_{2}{ }^{+}$. Adjusting the source operation condition, which is similar as that for $\mathrm{BF}_{2}{ }^{+}$production, can optimize the percentage of $\mathrm{O}_{2}^{+}$ions. By increasing the oxygen gas pressure, decreasing the RF power, and decreasing the gap between RF quartz antenna and extraction aperture, the percentage of $\mathrm{O}_{2}^{+}$increases. Over $90 \%$ of $\mathrm{O}_{2}{ }^{+}$can be achieved. For the negative oxygen ions as shown in Fig 5.6(b), much more $\mathrm{O}^{-}$ions were produced than $\mathrm{O}_{2}^{-}$ions.

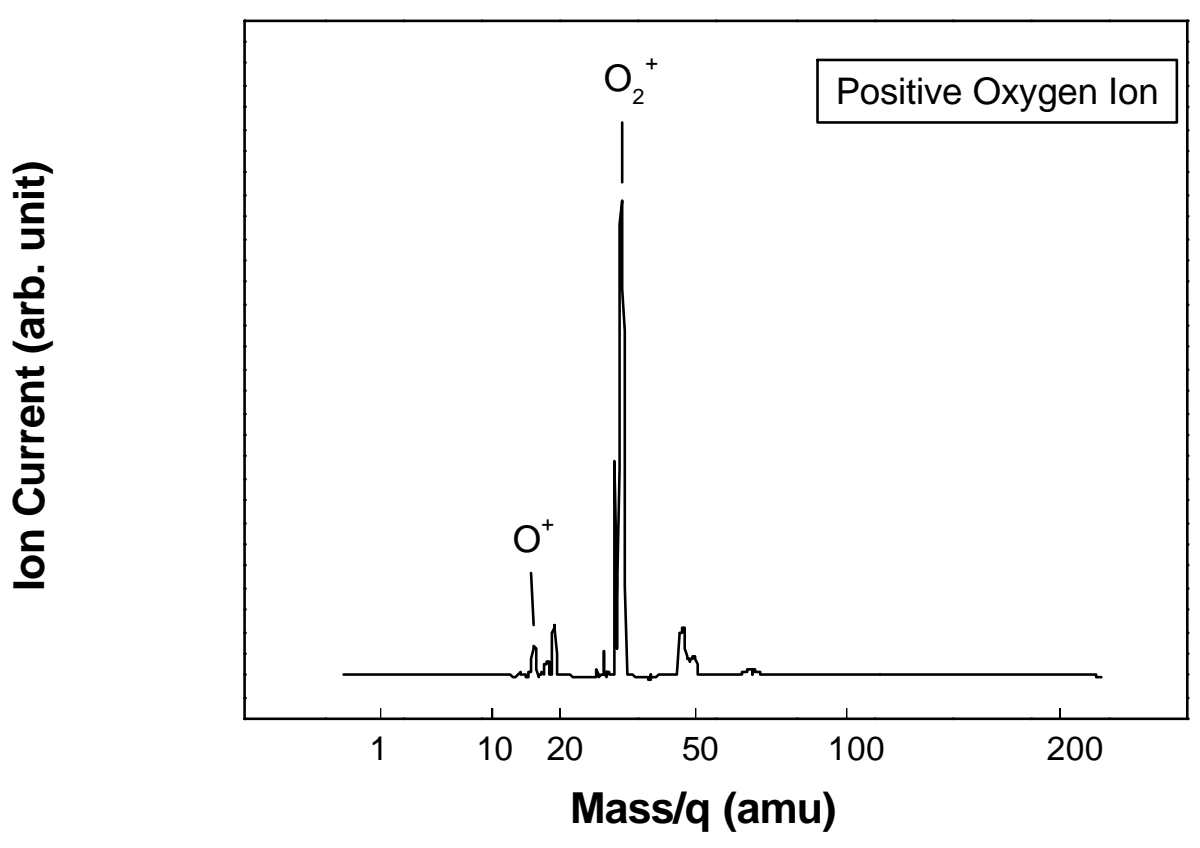

(a) 


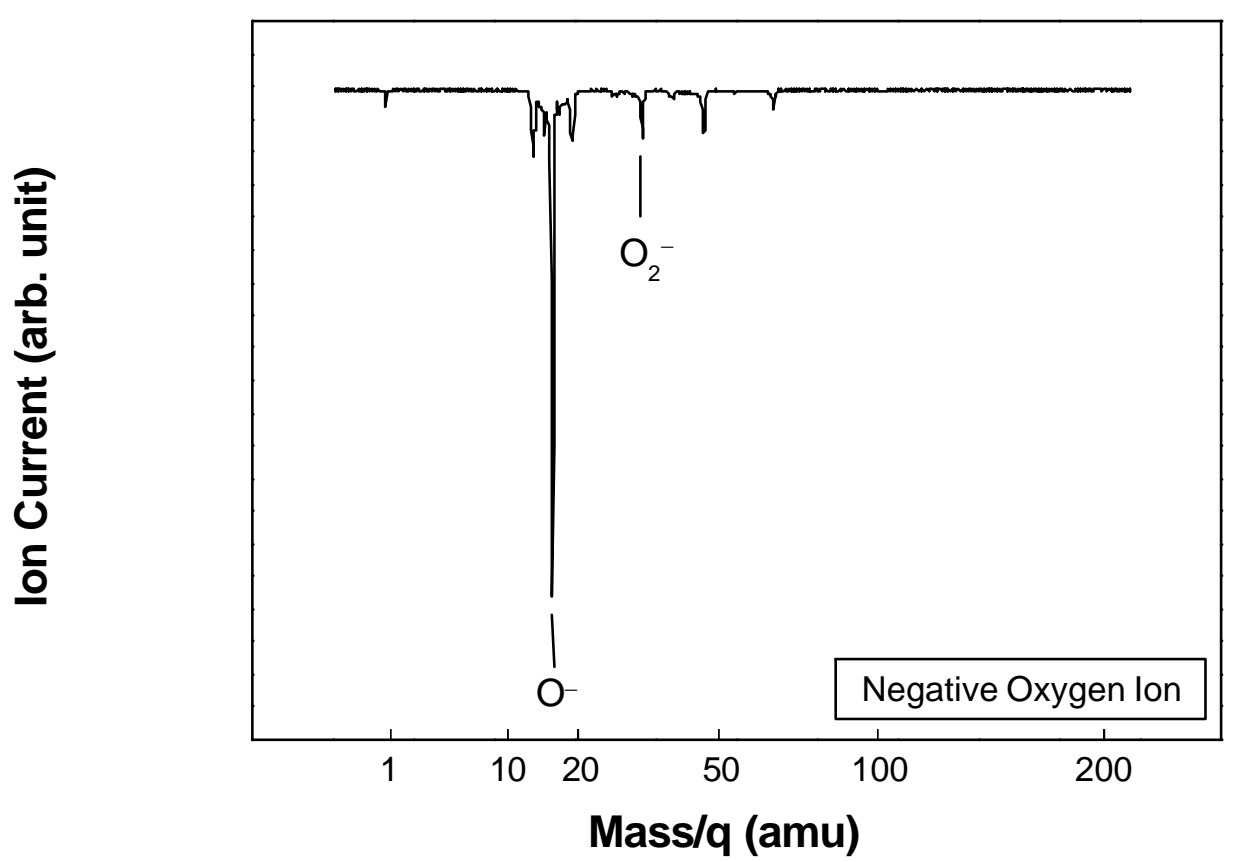

(b)

Figure 5.6 Mass spectra of Oxygen plasma. (a) positive ions; (b) negative ions.

\subsection{Direct write processes}

\subsubsection{Direct doping using boron and phosphorous beam}

The process of direct implantation is: implant focused $\mathrm{BF}_{2}^{+}$or $\mathrm{P}^{+}$ion beams directly into the silicon, and then activate the dopants by rapid thermal annealing. In order to achieve shallow $p$ - $n$ junction, low energy boron or phosphorus ions should be used so that the dopants won't penetrate too deep into the substrate. Figure 5.7 and Figure 5.8 shows the $\mathrm{TRIM}^{2}$ simulation results of implantation profile and ion distribution for 1.0 $\mathrm{keV} \mathrm{B}{ }^{+}$and $5 \mathrm{keV} \mathrm{P}^{+}$respectively. The energy of $\mathrm{BF}_{2}{ }^{+}$beam can be $4 \mathrm{keV}$, because when $\mathrm{BF}_{2}{ }^{+}$ion reaches $\mathrm{Si}$ substrate, it breaks up into one boron atom and two fluorine atoms. Boron atom only carries about $22 \%$ of the total energy. 


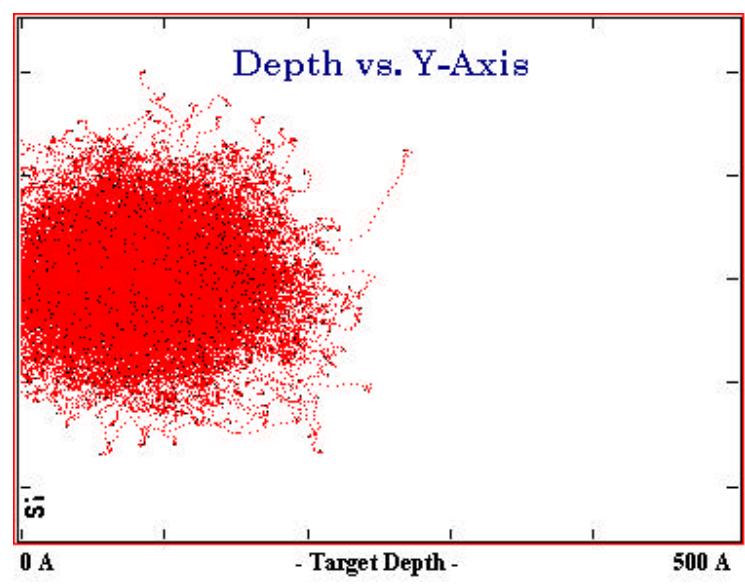

(a)

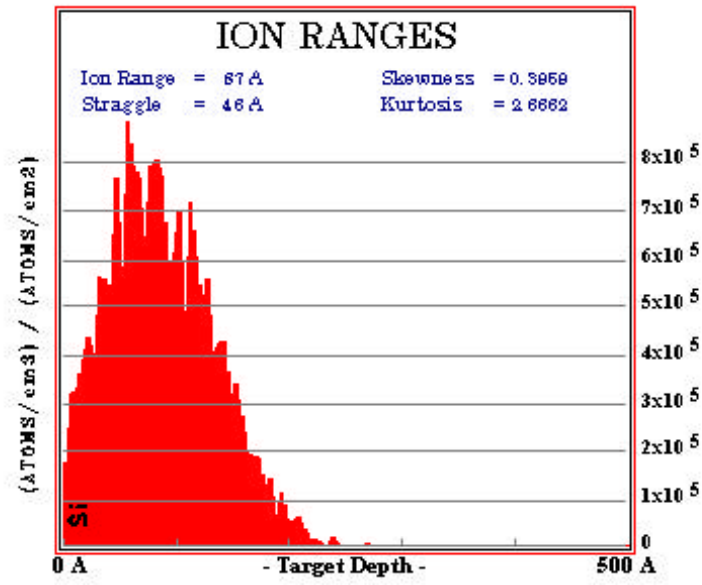

(b)

Figure 5.7 TRIM simulation results of $1.5 \mathrm{keV} \mathrm{B}$ B $^{+}$implanted into Si substrate. (a)Implantation profile, (b)Ion distribution.

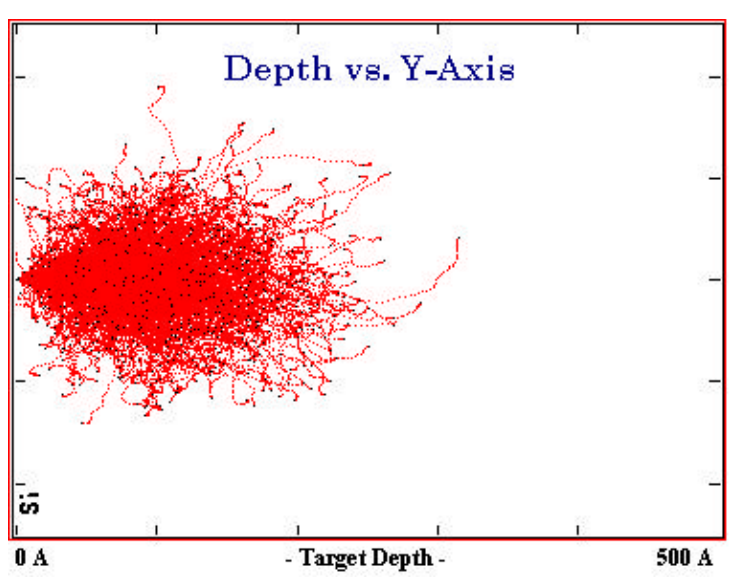

(a)

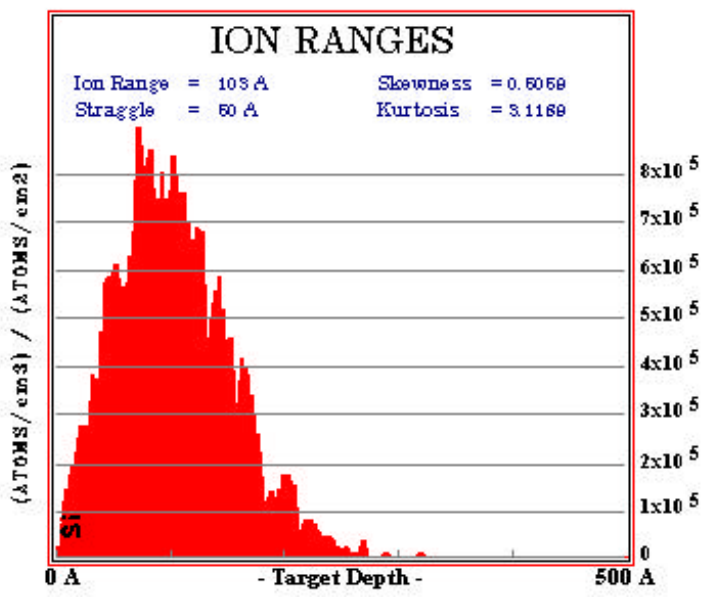

(b)

Figure 5.8 TRIM simulation results of $5 \mathrm{keV} \mathrm{P}^{+}$implanted into Si substrate.

(a)Implantation profile, (b)Ion distribution.

To confirm this process, $5 \mathrm{keV} \mathrm{P}^{+}$ions was implanted into a p-type silicon substrate, followed by a rapid thermal annealing at $975^{\circ} \mathrm{C}$ for 10 seconds. Rutherford Backscattering Spectrum shown in Fig. 5.9 was measured before the RTA. Phosphorus peak in the plot confirms the implantation. The dose derived is about $10^{16} / \mathrm{cm} 2$. The Hall effect measurement of the sample shows that the electron concentration is about $2.5 \times 10^{18}$ 
$\mathrm{cm}-3$ and electron mobility is around $200 \mathrm{~cm}^{2} / \mathrm{V} \cdot \mathrm{s}$. The activation is low because there is no oxide layer to cap the wafer, and phosphorus diffuses during RTA process.

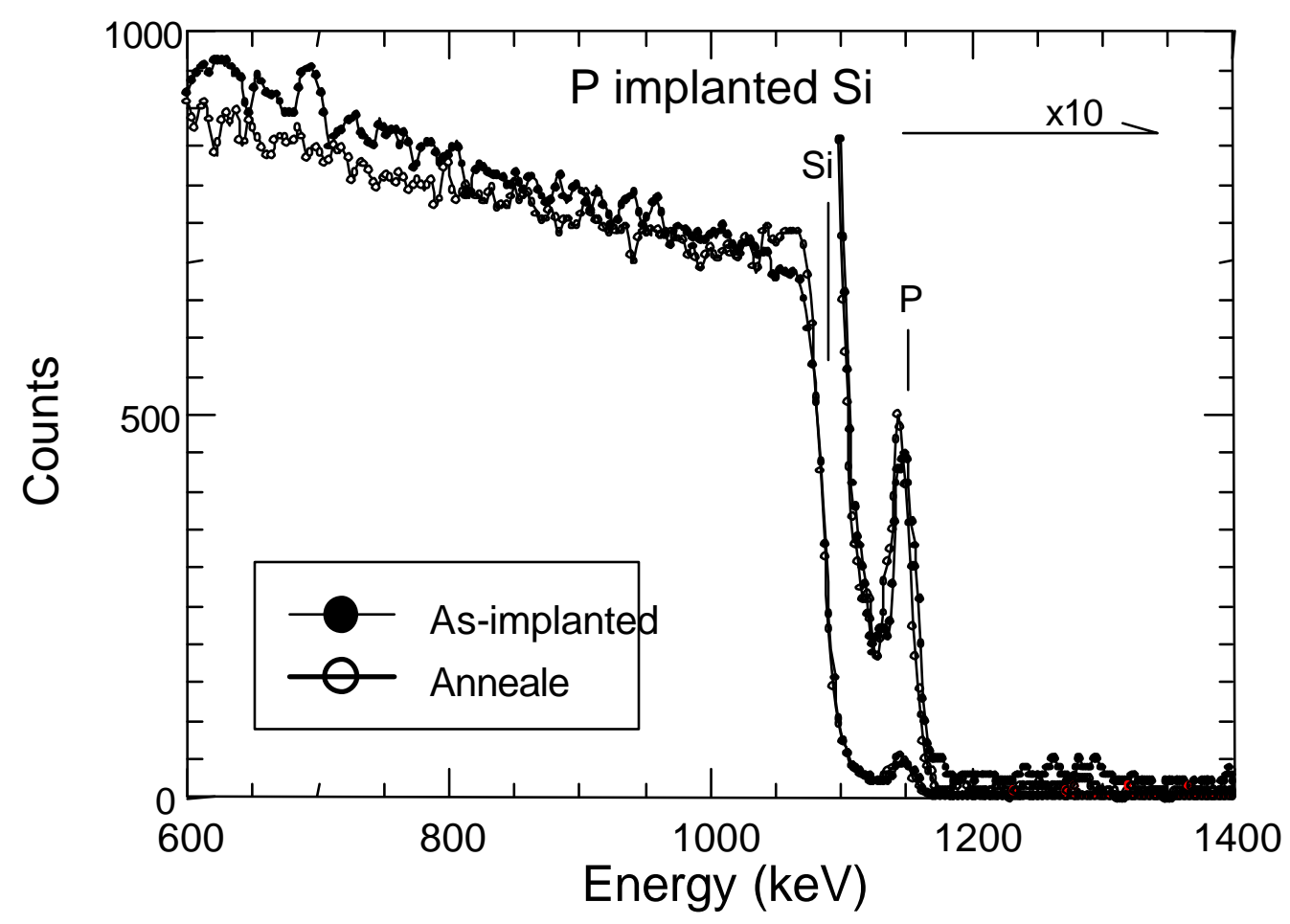

Figure 5.9 Rutherford backscattering spectrum of as-implanted and annealed Si wafer after doped with $5 \mathrm{keV} \mathrm{P}^{+}$ion beam.

\subsubsection{Surface modification using oxygen beam}

Selective oxidation of a Si film by AFM lithography has been demonstrated to be a viable technique for fabrication of $0.1 \mu \mathrm{m}$ devices. ${ }^{3}$ A thin $(3 \mathrm{~nm}$-thick) surface oxide serves as an etch mask for patterning of the Si film, which can subsequently be used as a mask to pattern underlying films. But the current form an AFM tip is only at pico-ampere range, the low throughput prevented it from application to massive device fabrication.

Similar to the low energy boron implantation, when $3 \mathrm{keV} \mathrm{O}_{2}^{+}$ion reaches $\mathrm{Si}$ substrate, it breaks up into two oxygen atoms. Each oxygen atom carries half of the total 
energy. Figure 5.10 shows the TRIM simulation results of implantation profile and ion distribution for $1.5 \mathrm{keV} \mathrm{O}^{+}$. In the simulation, around $20 \AA$ thick native oxide was taken into account.
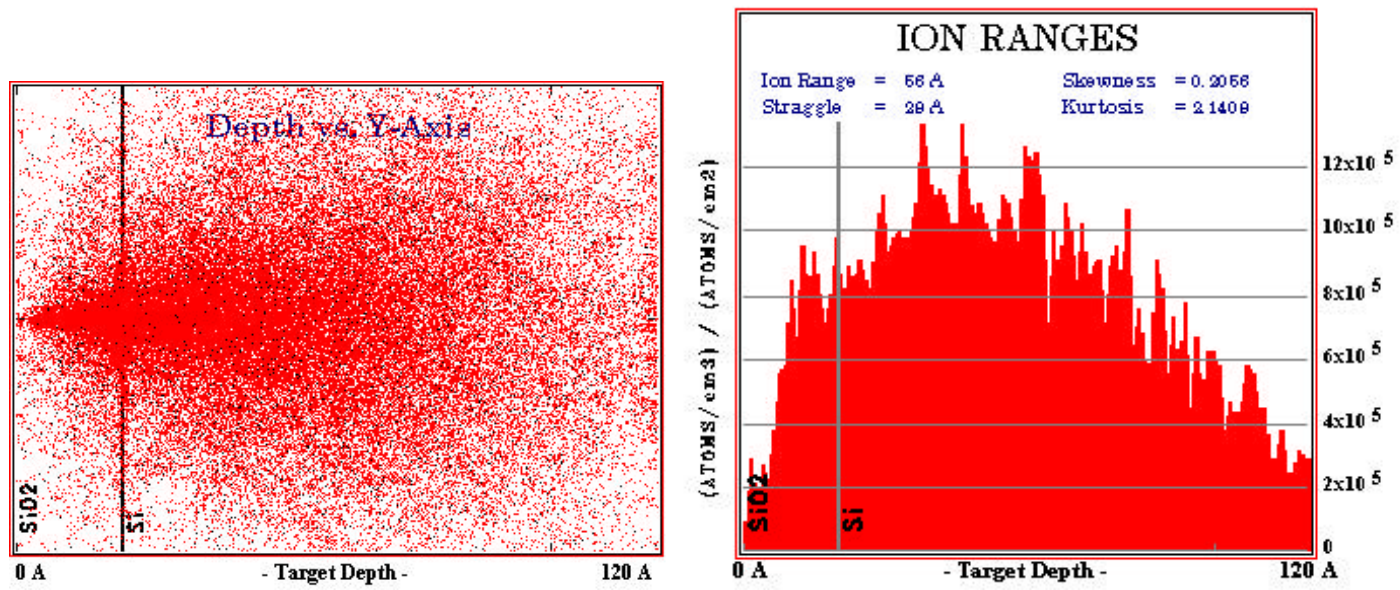

Figure 5.10 TRIM simulation results of $1.5 \mathrm{keV} \mathrm{O}^{+}$implanted into Si substrate. (a)Implantation profile, (b)Ion distribution.

The process of directly oxidizing silicon or polysilicon surface is as shown in Fig. 5.11. The test wafer was prepared with $1500 \AA$-thick poly-Si on top of a layer of thermal oxide, which is usually the etch stop in silicon etching process. Implant $\mathrm{O}_{2}{ }^{+}$ions at energy of $3 \mathrm{keV}$ to form a thin silicon dioxide layer, which acts as the hard mask in the following reactive-ion etching process to pattern poly-Si gate. 
a) Start with a bare Si wafer. Grow thermal oxide as etch stop. Deposit

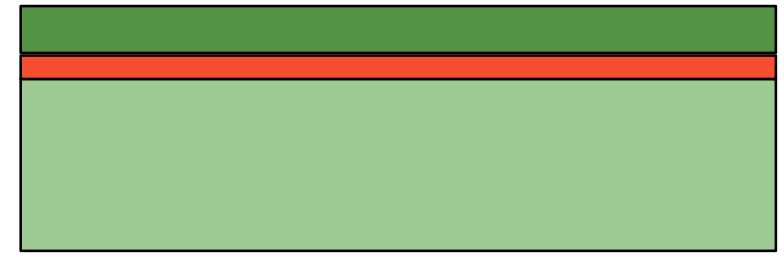

b) Expose the wafer to focused $\mathrm{O}_{2}{ }^{+}$beam. It forms $\mathrm{SiO}_{2}$ at the poly-Si surface.

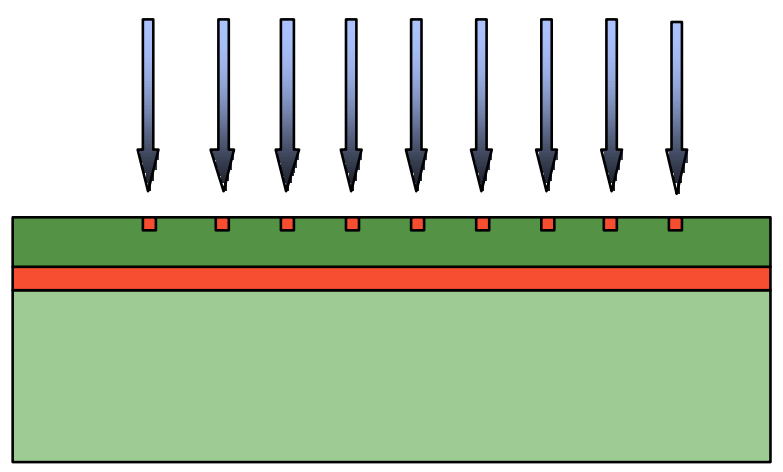

c) Use the formed $\mathrm{SiO}_{2}$ as a hard mask, pattern the poly-Si using dry etch.

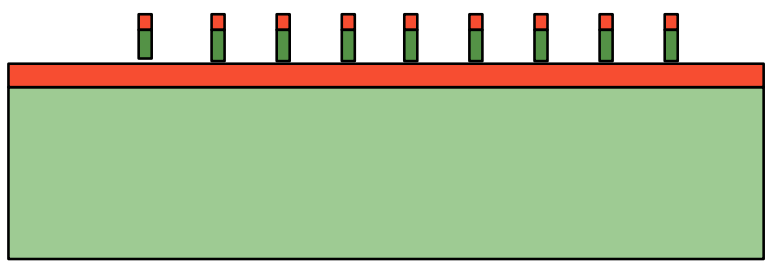

Figure 5.11 Process flow of direct poly-Si patterning using focused $\mathrm{O}_{2}{ }^{+}$beam.

In order to consume least oxide mask, limited native oxide breakthrough and silicon etching with highest $\mathrm{Si}_{\mathrm{SiO}}$ 2 selectivity is chosen. As shown in Table 5.1, in the first step, minimum RF power and etching time is used. The RF power applied on the top electrode is $70 \mathrm{~W}$, and $10 \mathrm{~W}$ on the bottom electrode. The gas flow of $\mathrm{CF}_{4}$ is $100 \mathrm{sccm}$, and the chamber pressure is kept around 13 mTorr. Only $5 \mathrm{sec}$ breakthrough time is enough to strip the native oxide. In the second step, an over-etch step in a conventional reactive-ion silicon etching process is adopted. The RF power applied on the top 
electrode is $250 \mathrm{~W}$, and the $120 \mathrm{~W}$ on the bottom electrode. The gas flow of $\mathrm{HBr}$ is 200 $\mathrm{sccm}$ and that of $\mathrm{O}_{2}$ is $5 \mathrm{sccm}$, and the chamber pressure is kept around $35 \mathrm{mTorr}$. PolySi film with $1500 \AA$ thickness can be etched within about 20 to 30 seconds.

Table 5.1 Recipe for poly-Si etch with high $\mathrm{Si}_{\mathrm{SiO}}$ selectivity.

\begin{tabular}{|c|c|c|}
\hline Step & Native oxide breakthrough & Si etch \\
\hline Pressure & 13 mTorr & 35 mTorr \\
\hline RF Power & $70 \mathrm{~W}$ (top), $10 \mathrm{~W}$ (bottom) & $250 \mathrm{~W}$ (top), $120 \mathrm{~W}$ (bottom) \\
\hline Gas & $\mathrm{CF}_{4}$ at $100 \mathrm{sccm}$ & $\mathrm{HBr}$ at $200 \mathrm{sccm}, \mathrm{O}_{2}$ at $5 \mathrm{sccm}$ \\
\hline Time & $5 \mathrm{sec}$ & About $20 \mathrm{sec}$ \\
\hline
\end{tabular}

Since no deflector is included in the focusing column, lines of $\mathrm{SiO}_{2}$ is formed simply by mechanically moving the wafer holder. Different dose is obtained by different scanning speed. Figure 5.12 and Figure 5.13 shows the patterned poly-Si line at two different doses and their corresponding profiles. In Figure 5.12, the scanning rate of the sample stage is $100 \mu \mathrm{m} / \mathrm{sec}$, and the oxygen dose is about $10^{15} \mathrm{~cm}^{-2}$. The line width of the poly-Si after etching is about $11 \mu \mathrm{m}$, and its thickness is about $1400 \AA$. In Figure 5.13, the scanning rate of the sample stage increases to $250 \mu \mathrm{m} / \mathrm{sec}$, and the oxygen dose is about $6 \times 10^{14} \mathrm{~cm}^{-2}$. The line width reduces to $9 \mu \mathrm{m}$ and its thickness is only $900 \AA$ A . Lower oxygen dose results in thinner oxide layer formed. With less hard mask thickness, thinner poly-Si is achieved. 


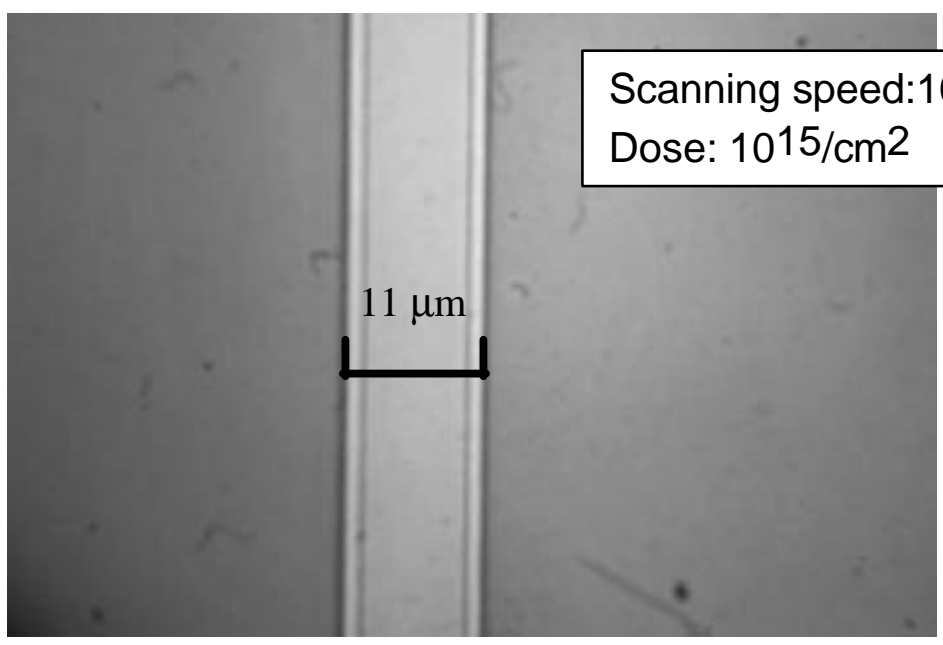

(a)

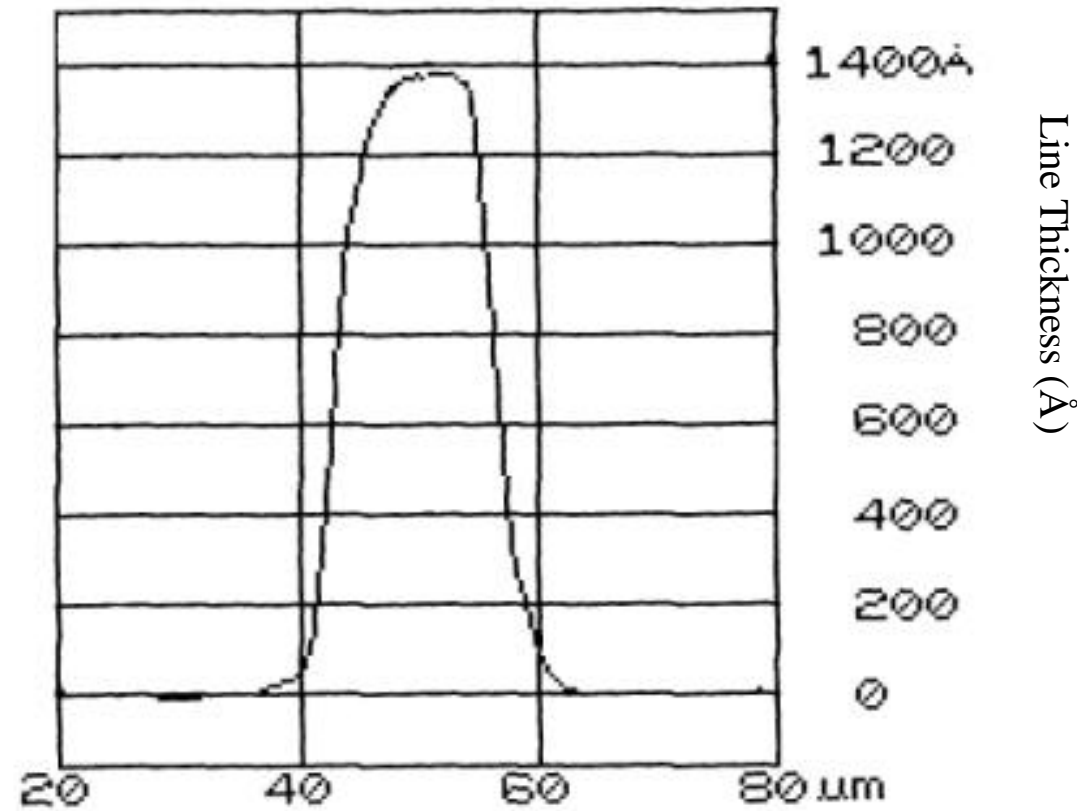

Line width $(\mu \mathrm{m})$

(b)

Figure 5.12 (a) Micrograph of patterned poly-Si features with focused $\mathrm{O}_{2}{ }^{+}$beam at dose of $10^{15} \mathrm{~cm}^{-2}$. (b)Profile of the poly-Si line. 


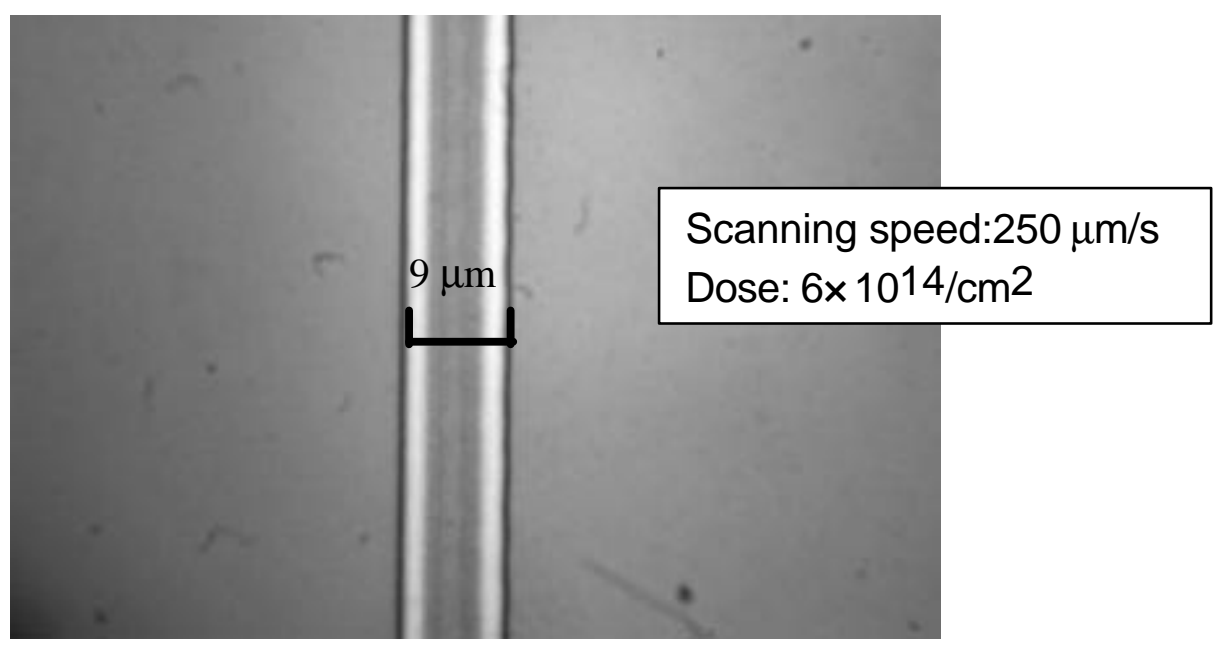

(a)

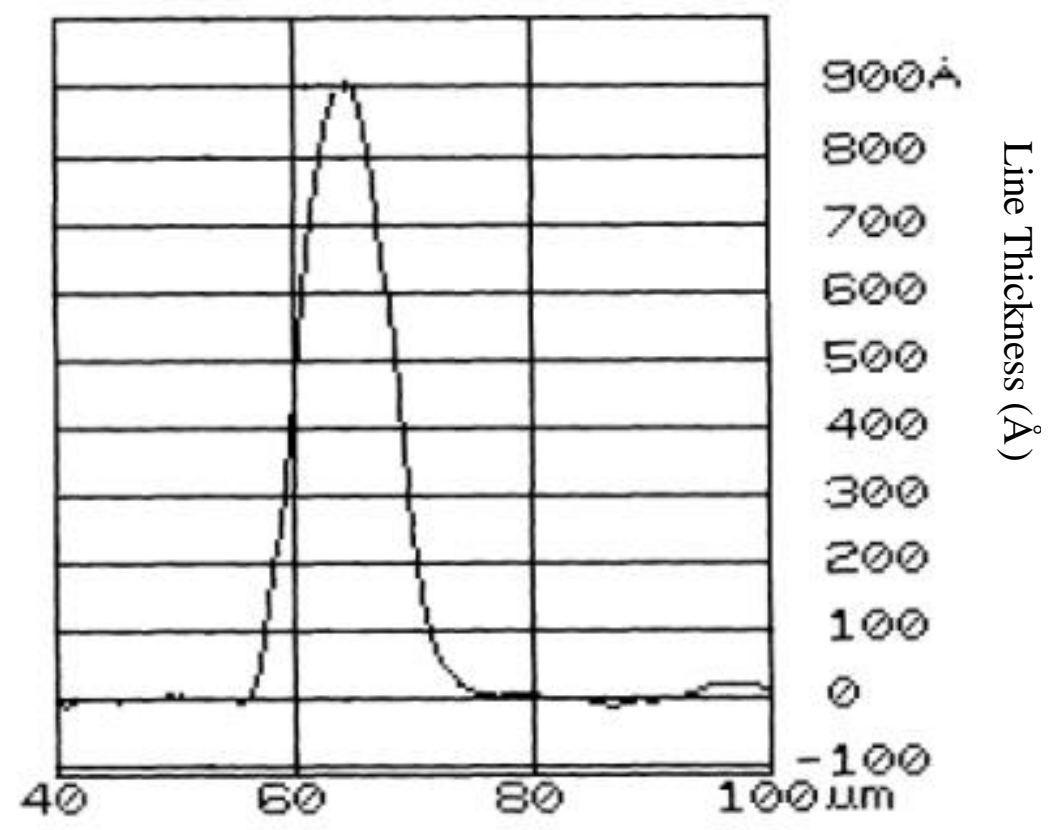

Line width $(\mu \mathrm{m})$

(b)

Figure 5.13 (a) Micrograph of patterned poly-Si features with focused $\mathrm{O}_{2}{ }^{+}$beam at dose of $6 \times 10^{14} \mathrm{~cm}^{-2}$. (b)Profile of the poly-Si line.

Poly SiGe is often used as gate material in MOSFET. Studies of direct pattering poly $\mathrm{SiGe}$ have also been performed. Since poly $\mathrm{SiGe}$ oxidizes faster than poly $\mathrm{Si}$, thicker 
native oxide layer is expected. Longer native oxide breakthrough and main silicon etching are necessary to avoid "black silicon islands". The modified recipe is shown in Table 5.2. In the breakthrough step, the RF power applied on the top electrode is changed to $200 \mathrm{~W}$, and $40 \mathrm{~W}$ on the bottom electrode. A 5-sec breakthrough time is needed to strip the native oxide. A 3-sec main etch step is added. The RF power is 300 $\mathrm{W}$ on the top and $150 \mathrm{~W}$ on the bottom. $\mathrm{Ch}$ flows at $50 \mathrm{sccm}, \mathrm{HBr}$ flows at $150 \mathrm{sccm}$, and the chamber pressure is kept around 12 mTorr. The over etch step is the same as that for poly-Si. Compare to poly-Si patterning, the oxygen dose for patterning is about twice as high. About $12 \mu \mathrm{m}$ wide, $1200 \AA$ thick poly- $\mathrm{Si}_{0.8} \mathrm{Ge}_{0.2}$ line is obtained with the scanning speed of $50 \mu \mathrm{m} / \mathrm{sec}$.

Table 5.2 Recipe for poly- $\mathrm{Si}_{0.8} \mathrm{Ge}_{0.2}$ etch with high $\mathrm{Si}_{\mathrm{SiO}}$ selectivity.

\begin{tabular}{|c|c|c|c|}
\hline Step & $\begin{array}{c}\text { Native oxide } \\
\text { breakthrough }\end{array}$ & Main etch & Over etch \\
\hline Pressure & 13 mTorr & $12 \mathrm{mTorr}$ & $35 \mathrm{mTorr}$ \\
\hline RF Power & $\begin{array}{c}200 \mathrm{~W} \text { (top), } \\
40 \mathrm{~W} \text { (bottom) }\end{array}$ & $\begin{array}{c}300 \mathrm{~W} \text { (top), } \\
150 \mathrm{~W} \text { (bottom) }\end{array}$ & $\begin{array}{c}250 \mathrm{~W} \text { (top), } \\
120 \mathrm{~W} \text { (bottom) }\end{array}$ \\
\hline Gas & $\mathrm{CF}_{4}$ at $100 \mathrm{sccm}$ & $\begin{array}{c}\mathrm{Cl}_{2} \text { at } 50 \mathrm{sccm} \\
\mathrm{HBr} \text { at } 150 \mathrm{sccm},\end{array}$ & $\begin{array}{c}\mathrm{HBr} \text { at } 200 \mathrm{sccm}, \\
\mathrm{O}_{2} \text { at } 5 \mathrm{sccm}\end{array}$ \\
\hline Time & $5 \mathrm{sec}$ & $3 \mathrm{sec}$ & About $20 \mathrm{sec}$ \\
\hline
\end{tabular}

\subsection{SOI MOSFET fabrication using maskless resistless ion beam lithography}

1. Start with a SOI wafer with $1000 \AA$ $\mathrm{Si}$ on 4000 Å buried oxide.

2. Thin down the silicon film by thermal oxidation. Pattern source and drain islands.
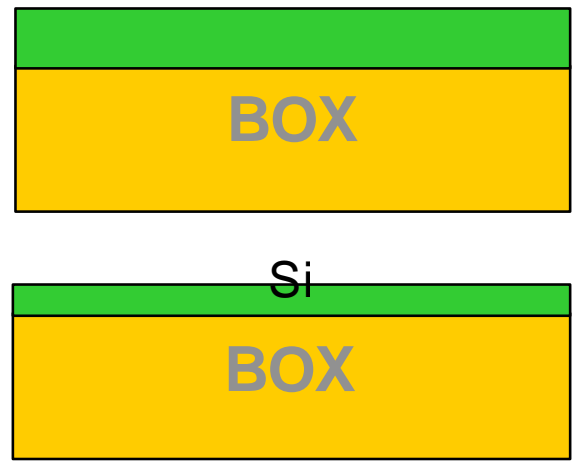
3. Grow thin gate oxide and deposit P-in-situ doped poly-Si.

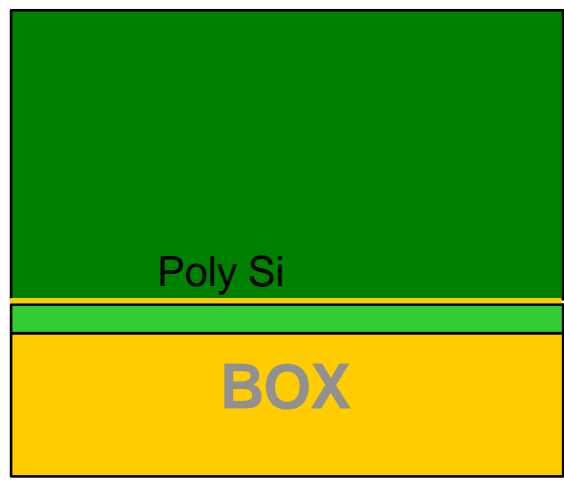

4. Selectively oxidize poly-Si surface

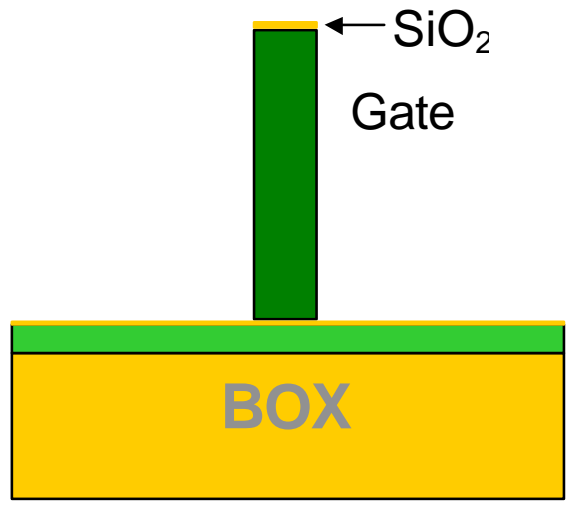
using focused $\mathrm{O}_{2}+$ beam, pattern poly-Si gate.

5. Selectively dope source and drain using focused $\mathrm{P}+$ beam, RTA, probe device.

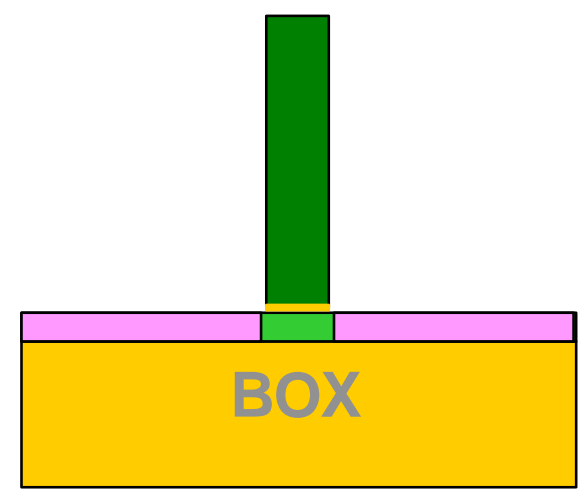

Figure 5.14 Process flow of SOI MOSFET fabrication using maskless, resistless ion beam lithography.

In order to demonstrate maskless, resistless ion beam lithography process, a SOI MOSFET is fabricated. The process flow is shown in Figure 5.14. A SOI wafer with $1000 \AA$ silicon film on top of $4000 \AA$ buried oxide is thinned down by thermal oxidation. 
After four cycles of oxidation at $950^{\circ} \mathrm{C}$ for 70 minutes followed by $\mathrm{HF}$ oxide etch, about $300 \AA$ of silicon is left. Source and drain areas are directly patterned using focused oxygen beam at dose of about $10^{14} / \mathrm{cm}^{2}$ (stage scanning speed of $500 \mathrm{um} / \mathrm{sec}$ ). About 80 $\AA$ of gate oxide is grown at $900^{\circ} \mathrm{C}$ for 12 minutes. About $1200 \AA$ of phosphorus in-situ doped poly-Si film is deposited on afterwards. Then, the wafer is exposed to focused oxygen beam to form oxide hard mask. After the gate is patterned, focused phosphorus ions is used to dope both source and drain area. Finally, rapid thermal annealing is performed to active the dopants, followed by the HF dip to remove any remaining oxide film. The pattered device is shown in Figure 5.15. During all the processes, neither mask nor resist is involved.

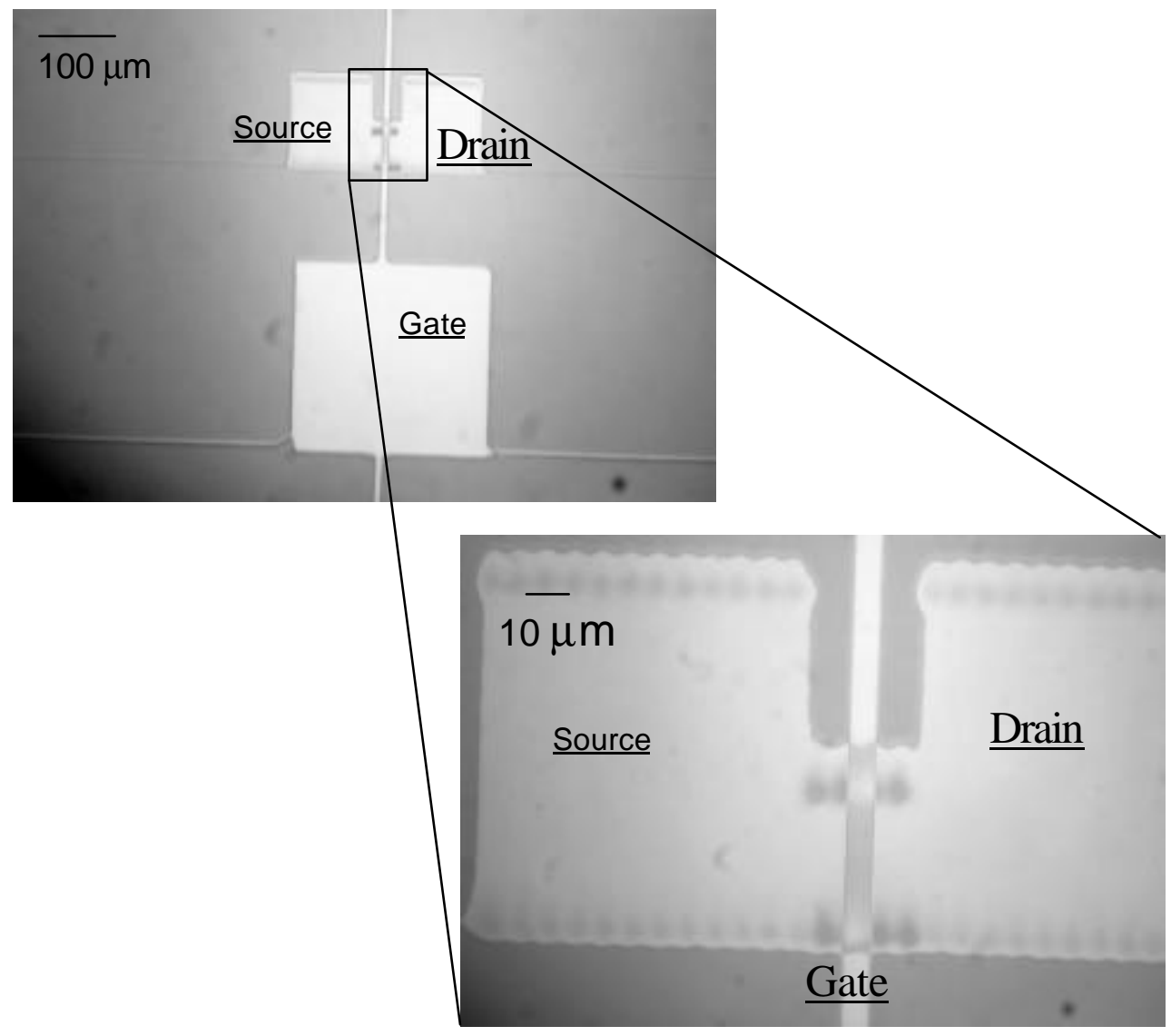

Figure 5.15 Micrograph of patterned SOI MOSFET. 


\section{References:}

1 J. Ishikawa, "Negative-ion sources for modification of materials (invited)", Rev. Sci. Instrum. 67, 1410(1996).

2 J. Ziegler, "The Stopping and Range of Ions in Matter", SRIM \& TRIM software, 2000.

3 S. C. Minne, H. T. Soh, Ph. Flueckiger, and C. F. Quate, 'Fabrication of $0.1 \mathrm{mu} \mathrm{m}$ metal oxide semiconductor field-effect transistors with the atomic force microscope", Appl. Phys. Lett. 66, pp.703-705, 1995. 


\section{Chapter 6}

\section{Multiple Focused Ion Beam System}

Exposure throughput is always an issue for both electron beam and ion beam lithography system. In order to process sixty 300-mm-diameter wafers per hour, multiple beamlets system is one of the promising solutions.

Figure 6.1 shows the schematic diagram of a multiple focused ion beam system. A multicusp plasma ion source is employed to generate the desired ion species, such as $\mathrm{O}_{2}{ }^{+}$, $\mathrm{BF}_{2}^{+}$, and $\mathrm{P}^{+}$etc. As mention in Chapter 2, this type of source can generate uniform and large area plasma, therefore, only one source is needed to generate multiple beams. A stack of electrodes with multiple apertures act as extractors and focusing lens to extract the beam from the source, accelerate to certain energy, and then focus to a small beam size at the target. Individual beamlet can be switched off simply be applying $+30 \mathrm{~V}$ on the extraction electrode relative to the source. ${ }^{1}$ The middle electrode of the Finzel lens can be split as shown in Fig 6.2 to act as a deflector. 


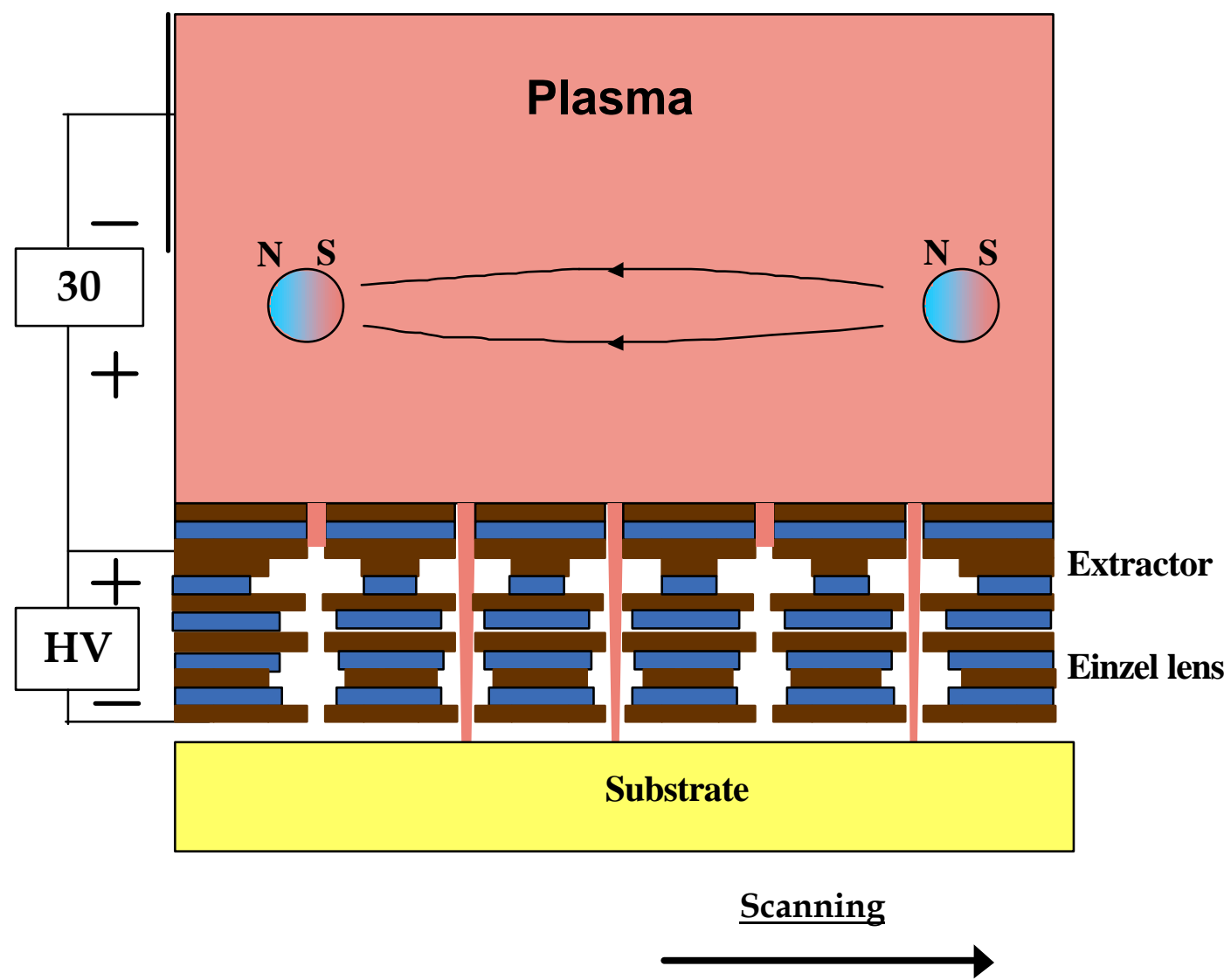

Figure 6.1 Schematic diagram a multiple focused ion beam system.

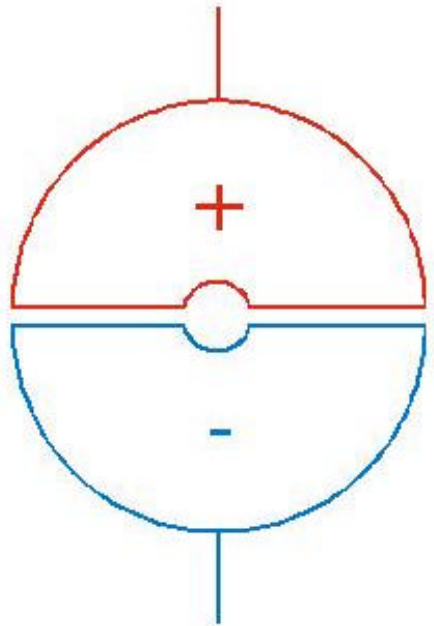

Figure 6.2 Deflector electrode. 
To the first order, the amount of current required to expose an entire wafer in an allotted time can be calculated using the following formula:

$$
I=\frac{\text { Area }_{\text {exp ose }} \cdot \text { Dose }}{\text { Time }_{\text {exp ose }}}
$$

For selective surface modification and direct doping, the dose requirement is on the order of $10^{15} / \mathrm{cm}^{2}$. In order to directly write a $300-\mathrm{mm}$ wafer (assuming $50 \%$ of the area is exposed) in 60 seconds, the total current of $0.94 \mathrm{~mA}$ will be required. The total number of columns needed depend on the amount of current that can be delivered to the target plane for each beamlet. As stated in Chapter4, for each beamlet, it is not difficult to achieve a current density of $10 \mathrm{~A} / \mathrm{cm}^{2}$ at the target. If the beam spot size is $50 \mathrm{~nm}$, the current available for each beamlet is $196 \mathrm{pA}$. Therefore, total of around 4 million columns are needed to fulfill the throughput. ${ }^{2}$

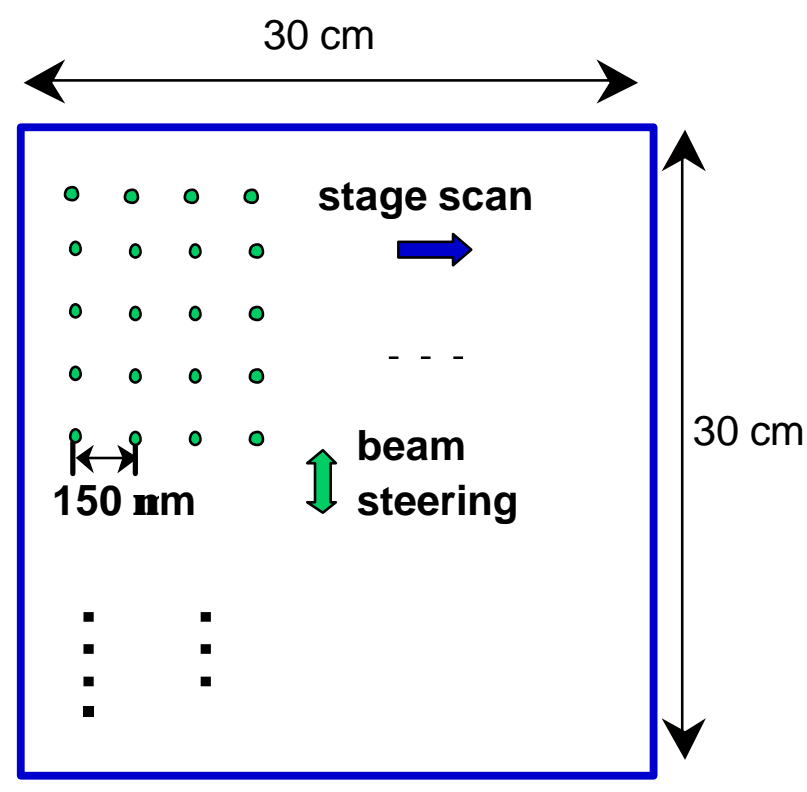

Figure 6.3 Layout of multiple beamlets. 
Fig 6.3 shows the layout of multiple beamlets. $2190 \times 2190$ beams are arranged in a way as shown in the plot. The gap between each adjacent beamlet is $150 \mu \mathrm{m}$, and 2190 beams cover a spatial dimension of $300 \mathrm{~mm}$. It turns out the each beamlet only needs to cover an area of $150 \mu \mathrm{m} \times 150 \mu \mathrm{m}$. Beams are deflected in one direction, and the substrate itself is scanned in the perpendicular direction. With an individual beam current of $196 \mathrm{pA}$, it will take approximately about $16 \mu$ s to expose a $50 \mathrm{~nm}$ spot. Each beamlet scans a total length of $150 \mu \mathrm{m}$ in $50 \mathrm{~nm}$ steps and will take $48 \mathrm{~ms}$ to complete a full sweep. The beam switching rate must be on the order of $62.5 \mathrm{KHz}$ in order to turn the beam on or off with each $16 \mu$ s exposure.

All the data rates calculated above seem to be achievable. However, still a lot of challenges exist to fulfill multiple beamlets, which are listed as follows:

1. Four million is a huge number. Fabricating a stacking column with such a huge amount of apertures is not easy.

2. To ensure good alignment for all the four million columns is a tough job, unless self-align fabrication technique is adopted.

3. The aperture size of each electrode is limited to at least $75 \mu \mathrm{m}$, which is half of the distance of the adjacent beams. Therefore new column ion optics design that takes this into account needs to be performed.

4. A lot of efforts have to be devoted in developing the circuits to control the beam switching and beam steering.

All these indicate that it will be more practical if the total number of beamlets can be reduced. If the focused current density of each beamlet increases by a factor of hundred, the total number of beamlets will reduce to around 40000. In this case, with an individual 
beam current of $19 \mathrm{nA}$, it will take approximately about $160 \mathrm{~ns}$ to expose a $50 \mathrm{~nm}$ spot. The beam switching rate must be on the order of $6.25 \mathrm{MHz}$ in order to turn the beam on or off with each $160 \mathrm{~ns}$ exposure. The gap between each adjacent beamlet is $1.5 \mathrm{~mm}$ Each beamlet scans a total length of $1.5 \mathrm{~mm}$ in $50 \mathrm{~nm}$ steps and will take $4.8 \mathrm{~ms}$ to complete a full sweep. Larger distance between two adjacent beamlets relieve some of the constraint of ion optics design, however faster control circuit and substrate stage with faster scanning speed are required. A comparison of these two cases is summarized in Table 6.1.

Table 6.1 Comparison of two microcolumn arrays.

\begin{tabular}{|c|c|c|}
\hline & $2190 \times 2190$ array & $219 \times 219$ array \\
\hline Current of each beam & $196 \mathrm{pA}$ & $19.6 \mathrm{nA}$ \\
\hline Time to expose each pixel & $16 \mu \mathrm{s}$ & $160 \mathrm{~ns}$ \\
\hline Switching rate & $62.5 \mathrm{kHz}$ & $6.25 \mathrm{MHz}$ \\
\hline Distance of adjacent beam & $150 \mu \mathrm{m}$ & $1.5 \mathrm{~mm}$ \\
\hline
\end{tabular}

\section{References:}

${ }^{1}$ V. V. Ngo, B. Akker, K. N. Leung, I. Noh, and S. Wilde, "Demonstrations of Electronic Pattern Switching and 10x pattern demagnification in a maskless micro-ion-beam reduction lithography system", $46^{\text {th }}$ International Conference on Electron, Ion, and Photon Beam Technology and Nanofabrication, Anaheim, CA, May28-31, 2002.

${ }^{2}$ K. L. Scott, "Maskless ion beam lithography using microcolumn arrays", Master Thesis, spring 2001. 


\section{Chapter 7}

\section{Conclusion}

In conclusion, maskless, resistless ion beam lithography is one of the promising techniques for sub-100nm lithography. A comprehensive study has been carried out in this thesis work in the following topics: choice of ion sources, improvement of the brightness of the ion source, design and testing of an all-electrostatic accelerating column for ion extraction and focusing, process development for direct doping using $\mathrm{BF}_{2}^{+}$and $\mathrm{P}^{+}$ ions, process development of direct patterning of silicon film using focused oxygen beam, and fabrication of a SOI MOSFET using maskless, resistless patterning technique.

\subsection{Contributions}

Multicusp plasma ion source has been shown to be promising for maskless, resistless ion beam lithography. It can generate uniform, large area plasma so that only one source is needed to produce thousands or millions of beams to enhance throughput. It can generate ion beams of various elements, which makes resistless processing possible. The 
low axial energy spread results in low chromatic aberration in ion optics, which eases the design of low energy ion beam focusing lens.

The mass spectra of both positive and negative ions have been measured for phosphorus, $\mathrm{BF}_{3}$ and oxygen multicusp plasmas. More varieties of negative ions were observed in both the Phosphorus plasma and $\mathrm{BF}_{3}$ plasmas. Over $90 \%$ of $\mathrm{P}^{+}$ions have been achieved. Increasing the gas pressure and decreasing the source power can maximize the production of $\mathrm{BF}_{2}{ }^{+}$and $\mathrm{O}_{2}{ }^{+}$. With optimization of source operation parameters, around $85 \%$ of $\mathrm{BF}_{2}{ }^{+}$and over $90 \%$ of $\mathrm{O}_{2}{ }^{+}$have been achieved.

The measured spectral brightness of this ion source is better than $10^{4} \mathrm{~A} / \mathrm{cm}^{2} \mathrm{ASrAeV}$ at beam energy of $600 \mathrm{eV}$, which is about two orders of magnitude higher than that of the conventional plasma sources. The brightness of the ion beam extracted from a multicusp ion source can be high if it is operated in the inductively coupled mode and if the beam extraction system is optimized to reduce aberrations. For a $7.5-\mathrm{cm}$-diameter source with a $50-\mu \mathrm{m}$ counter-bored extraction aperture, the measured brightness $\left(440 \mathrm{~A} / \mathrm{cm}^{2} \mathrm{Sr}\right)$ is significantly higher than in previously reported work. Additional increases in RF power as well as improved source design are being investigated to further improve the brightness in order to meet the requirement of $1000 \mathrm{~A} / \mathrm{cm}^{2} \mathrm{Sr}$ for high-throughput maskless lithography.

An all-electrostatic accelerator column has been designed to extract $\mathrm{O}_{2}^{+}$ions from the source and accelerated to $3 \mathrm{keV}$. $73 \mathrm{nA}$ of $\mathrm{O}_{2}{ }^{+}$ion beam can be extracted from the source using such a column. Numerical analyses using both IGUN and Munro's code have shown that the demagnification factor of this two-lens system is $10 \times$, however, due to 
aberration limitation, the final beam spot size is around $16 \mu \mathrm{m}$ (corresponding to a $3 \times$ demagnification). Experiments show that the beam spot size from $20 \%$ to $80 \%$ is around $25 \mu \mathrm{m}$, which matches with the simulation results very well. Further reducing the beam half angle by placing a limiting aperture between the two lenses can decrease the aberration significantly.

It has been demonstrated that focused $\mathrm{P}^{+}$ions can be directly implanted into silicon at dose of $10^{16} \mathrm{~cm}^{-2}$, resulting electron density of $2.5 \times 10^{18} \mathrm{~cm}^{-3}$ and mobility of $200 \mathrm{~cm}^{2} / \mathrm{V} \cdot \mathrm{s}$. The process for direct patterning using focused $\mathrm{O}_{2}{ }^{+}$ion beams has also been demonstrated. At a dose of $10^{15} / \mathrm{cm}^{2}$, focused $\mathrm{O}_{2}{ }^{+}$ion beams can be used to selectively form a thin surface oxide film, which serves as a hard mask for patterning the underlaying Si film. A SOI MOSFET device has been successfully fabricated using the maskless, resistless lithography to pattern gate electrode and form source/drain region.

A multiple-beam system can be built by stacking multi-aperture, electrode-insulator structure so that each beam is accelerated with the same electrode potentials. Parallel processing with multiple beams can greatly enhance the throughput of a FIB lithography system.

\subsection{Suggestions for future work}

Much remains to be investigated and improved. Further improvement of column design is needed to achieve smaller beam spot size: adding a limiting aperture to reduce aberration, and fabricating an extraction aperture of $5 \mu \mathrm{m}$ and smaller. Brightness needs to be measured for the source with external antenna. Switchable extractor system needs to 
be investigated to demonstrate multiple beam feasibility. Beam-to-beam interactions must be analyzed to make sure no cross talk between adjacent beamlets. Other applications, for example, copper deposition and removal, can be investigated using maskless, resistless ion beam systems. 